
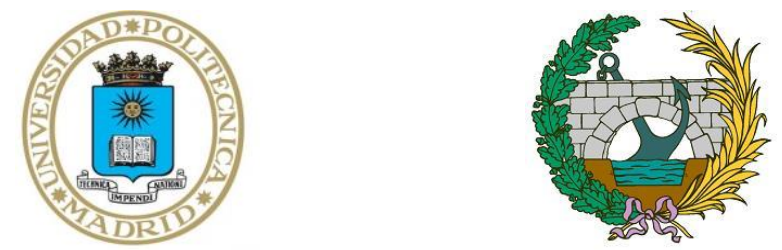

Universidad Politécnica de Madrid

Escuela Técnica Superior de Ingenieros de Caminos, Canales y Puertos

\title{
RAYLEIGH WAVES IN NONLINEAR ELASTICITY
}

Doctoral Thesis

Nam Nguyen Thi

Madrid, November 2016 

Departamento de Mecánica de Medios Continuos

y TEORÍA DE EsTruCturas

Escuela Técnica Superior de Ingenieros de

Caminos, Canales y Puertos

\title{
RAYLEIGH WAVES IN NONLINEAR ELASTICITY
}

Submitted in PARTial SATISFACTION OF THE

REQUIREMENTS FOR THE DEGREE

$\mathrm{OF}$

Doctor of Philosophy

\author{
A U THOR \\ Nam Nguyen Thi \\ S U P E R VISOR \\ Professor José Merodio
}


The composition of the text has been made using ATEX, Matlab, Maple.

Madrid 2016

Author; Nam Nguyen Thi

Email; namtnyb@gmail.com 
Committee appointed by the Rector of Universidad Politécnica de Madrid, on..... of ..... 2016.

President: D. Alejandro Pérez Caldentey

Vowel: D. Javier Rodriguez Soler

Vowel: D. Manuel de León Rodríguez

Vowel: D. Raymond William Ogden

Secretary: D. Javier Segurado Escudero

Defended on 29 of November 2016 at E.T.S. de Ingenieros de Caminos, Canales y Puertos, Universidad Politécnica de Madrid

Qualification:

PRESIDENT

VOWELS 


\section{Publications}

The following scientific articles have been derived from this work

- P.C. Vinh, J. Merodio, T.T. Hue, N. Nguyen, Non-principal Rayleigh waves in deformed incompressible transversely isotropic elastic halfspaces, IMA J. Appl. Math. 79 (2014) 915-928.

- N. T. Nam, J. Merodio, P. C. Vinh, The secular equation for nonprincipal Rayleigh waves in deformed incompressible doubly fiberreinforced nonlinearly elastic solids, Int. J. Non-Lin. Mech. 84 (2016) 23-30.

- N.T. Nam, J. Merodio, R.W. Ogden, P.C. Vinh, The effect of initial stress on the propagation of surface waves in a layered half-space, Int. J. Solid. Struct. 88-89 (2016) 88-100.

and the following conference proceeding

- N.T. Nam, J. Merodio, R.W. Ogden, P.C. Vinh, Effect of initial stress on the propagation of Rayleigh surface waves in a half space with a layer. EUROMECH Colloquium 551, Mechanics of Fibre-Reinforced Materials: Theory and Applications. University of Nottingham, 2nd5th of September 2013. 
This thesis studies Rayleigh waves in a nonlinear elastic continuum.

Firstly, an explicit expression of the secular equation for non-principal Rayleigh waves in incompressible, transversely isotropic, pre-stressed elastic half-spaces is obtained. The free surface coincides with one of the principal planes of the primary pure homogeneous strain, but the surface wave is not restricted to propagate in a principal direction. Results are given, for illustration, in respect of the so-called reinforcing models. Furthermore, exact formulas for the Rayleigh wave speed are given for two cases in which the propagation direction of the wave and the fiber reinforcement direction are restricted to coincide with a principal direction of the primary strain.

Secondly, the explicit and implicit secular equations for the speed of a (surface) Rayleigh wave propagating in a pre-stressed, doubly fiberreinforced incompressible nonlinearly elastic half-space are obtained. One of the principal planes of the primary pure homogeneous strain coincides with the free surface while the surface wave is not restricted to propagate in a principal direction. Results are illustrated with numerical examples. In particular, an isotropic material reinforced with two families of fibers is considered. Each family of fibers is characterized by defining a privileged direction. Furthermore, the fibers of each family are located throughout the half space and run parallel to each other and perpendicular to the depth direction.

Finally, the propagation of small amplitude surface waves guided by a layer with a finite thickness on an incompressible half-space is studied. The layer and half-space are both assumed to be initially stressed. The combined effect of initial stress and finite deformation on the speed of Rayleigh waves is analyzed and illustrated graphically. With a suitable simple choice of constitutive law that includes initial stress, it is shown 
that in many cases, as is to be expected, the effect of a finite deformation (with an associated pre-stress) is very similar to that of an initial stress (without an accompanying finite deformation). However, by contrast, when the finite deformation and initial stress are considered together independently with a judicious choice of material parameters different features are found that do not appear in the separate finite deformation or initial stress situations on their own. 
Esta tesis estudia la propagación de ondas Rayleigh en sólidos elásticos no lineales.

En primer lugar, se obtiene la expresión explícita de la ecuación secular para ondas de Rayleigh no principales en semiespacios elásticos incompresibles, transversalmente isotrópicos y con una tensión inicial. La superficie libre coincide con uno de los planos principales de la puramente homogénea deformación principal, pero la onda de superficie puede propagarse en direcciones diferentes a la de la dirección principal. Se proporcionan resultados para los denominados modelos de refuerzo. Asimismo, se proporcionan las formulas exactas de la velocidad de las ondas de Rayleigh para dos casos en los cuales la dirección de la propagación de la onda y la dirección de las fibras de refuerzo están coaccionadas a coincidir con la dirección principal de la deformación primaria.

Seguidamente, se obtienen las ecuaciones explícitas e implícitas para determinar la velocidad de ondas de Rayleigh (de superficie) que se propagan en semiespacios incompresibles no elásticos con doble fibra de refuerzo, con tensiones iniciales. Uno de los planos principales de la deformación primaria puramente homogénea coincide con la superficie libre mientras que la onda de superficie puede propagarse en direcciones diferentes a la de la dirección principal. Los resultados se ilustran con ejemplos numéricos. En particular, se considera un material isotrópico reforzado con dos familias de fibras. Cada familia de fibras se define mediante una dirección particular. De igual manera, las fibras de cada familia se consideran esparcidas a lo largo del semiespacio y son paralelas entre sí y perpendiculares con la vertical del semiespacio. Finalmente, se estudia la propagación de ondas de superficie de pequeñas amplitudes guiadas por una capa con un espesor finito en un semiespacio 
incompresible. Se supone que tanto la capa como el semiespacio tienen una tensión inicial. Se analiza el efecto combinado de la tensión inicial y las deformaciones finitas en la velocidad de las ondas de Rayleigh y luego se representa este efecto gráficamente.

Con una adecuada y simple elección de leyes constitutivas, que tienen en cuenta la tensión inicial, se muestra que en muchos casos el efecto de una deformación finita (con una tensión inicial asociada) es muy similar al de una tensión inicial (sin una deformación finita asociada). Sin embargo, en contraste, cuando la deformación finita y la tensión inicial se consideran al mismo tiempo con una elección juiciosa de los parámetros materiales, se observan características que no pueden ser apreciadas mediante un análisis separado de las deformaciones finitas o de las tensiones iniciales. 


\section{ACKNOWLEDGEMENT}

This thesis has been carried out thanks to the support and collaboration of many people and organizations to which I express my sincere gratitude.

In the first place, I wish to thank the supervisor of this work, Prof. José Merodio, who, throughout all of these years, has been a generous source of inspiration and help. His guidance and assistance cannot be overestimated.

I wish to acknowledge the support and help that I received from Prof. Raymond Ogden, Prof. Pham Chi Vinh, Prof. Michel Destrade, Prof. Davide Bigoni and Dr. Francesco Dal Corso that have been generous enough to share their time and genius.

This work has been financially supported by a scholarship sponsored by the Vietnamese Ministry of Education, by the Vietnam National Foundation for Science and Technology Development (NAFOSTED) under the grant no. 107.02-2014.04 and from the Ministry of Science in Spain under the project references DPI2011-26167 and DPI2014-58885-R.

Similarly, I wish to thank the many friends that have accompanied me in this journey, in particular I thank Ammar Alhayani, Mustapha El Hamdaoui, Sergio Blancas Saiz, and Pedram Manouchehri, their friendship and encouragement were vital in the completion of this goal.

I thank the love and support from my family, in particular my parents, my brother and sisters. 
Finally, I wish personally thank you.

To my husband, Freddy Ariñez, thank you for your support throughout this process. Thank you for always reminding me of my worth whenever I have doubted. Thank you for being the husband that has never stood in the way of any of my dreams yet you have always supported them.

To my little baby, Clara Trang, mommy loves you. Nothing could ever make me love you any less. 
Abstract

viii

1 Introduction

2 Rayleigh waves in incompressible materials reinforced with one fiber

2.1 Introduction . . . . . . . . . . . . . . 5

2.2 Material model and equations of motion . . . . . . . 6

2.2.1 Material model . . . . . . . . . . . . . . . 6

2.2.2 Linearized equations of motion: Incremental equations 8

2.3 Surface waves . . . . . . . . . . . . . . . . . . . . 11

2.3.1 The Stroh formulation . . . . . . . . . . . . 11

2.3.2 Implicit and explicit secular equation . . . . . . . . 12

3 Rayleigh waves in incompressible materials reinforced $\begin{array}{lr}\text { with two fibers } & 19\end{array}$

3.1 Introduction . . . . . . . . . . . . . . . . . . . 19

3.2 Basic equations . . . . . . . . . . . . . . . . . 20

3.2 .1 Kinematics . . . . . . . . . . . . . . . . 20

3.2.2 Material model . . . . . . . . . . . . . . . . 21

3.2.3 Linearized incremental equations of motion . . . 22

3.3 Surface waves . . . . . . . . . . . . . . . 26

3.3.1 The Stroh formulation . . . . . . . . . . . . . 27

3.3.2 Implicit secular equation . . . . . . . . . . . . 28

3.3.3 Explicit secular equation . . . . . . . . . . 29

3.4 Numerical results . . . . . . . . . . . . . . . . . . . . 30

4 Surface waves in a layered half-space with initial stress $\quad 39$

4.1 Introduction . . . . . . . . . . . . . . . . . 39

4.2 Basic equations . . . . . . . . . . . . . . . 41

4.2 .1 Kinematics and stress . . . . . . . . . . . 41

4.2.2 The strain-energy function . . . . . . . . . . . 42

4.2.3 Invariant formulation . . . . . . . . . . . . . . . 43

4.2.4 Plane strain specialization . . . . . . . . . . . 45

4.3 Incremental equations . . . . . . . . . . . . . . . 45 
4.3 .1 Plane strain case . . . . . . . . . . . . . 48

4.4 Plane incremental motions . . . . . . . . . . . . . . . . . 49

4.5 Surface waves in a layered half-space . . . . . . . . . . . . 50

4.6 Numerical illustrations . . . . . . . . . . . . . . . . . 58

$\begin{array}{lll}5 & \text { Conclusions } & 73\end{array}$

$\begin{array}{ll}\text { appendix } & 76\end{array}$

A Kinematics and geometry . . . . . . . . . 77

A.1 Combined extension, inflation and torsion . . . . 78

B Equilibrium and residual stress . . . . . . . . . . 79

C Constitutive laws . . . . . . . . . . . . . . . 80

C.1 Invariant formulation . . . . . . . . . . . 81

D Application to combined extension, inflation, torsion . . . 82

D.1 Equilibrium and boundary load . . . . . . . . 83

E Two simple models with residual stress . . . . . . . . . . 84

F Linear stability analysis . . . . . . . . . . . . 85

F.1 Definition of the incremental deformation . . . . 85

F.2 Stroh formulation . . . . . . . . . 87

$\begin{array}{ll}\text { Bibliography } & 102\end{array}$

$\begin{array}{ll}\text { List of figures } & 103\end{array}$

$\begin{array}{ll}\text { List of tables } & 108\end{array}$ 

This work follows previous works in the context of nonlinear elsticity trying to extend works from linear elasticity in different contexts including fiber reinforced failure and constituive modeling (see the references [1][19]). The examples of nonlinear elasticity analysis in different areas can also be found in [1]-[30].

Elastic surface waves, discovered by Rayleigh [31] more than 120 years ago for compressible isotropic elastic solids, have been studied extensively and exploited in a wide range of applications including seismology, acoustics, geophysics, telecommunications and material science, among others. In addition, the study of surface waves traveling along the free surface of an elastic half-space affects many aspects of modern life, stretching from mobile phones through the study of earthquakes, as addressed by Adams et al. [32].

The Rayleigh wave existence and uniqueness problem has been resolved with the aid of the Stroh formalism [33; 34] even for an anisotropic elastic half-space, see Barnett and Lothe [35-37], Chadwick and Smith [38] and Ting [39]. Based on an identity for the surface-impedance matrix, $\mathrm{Fu}$ and Mielke [40], Mielke and $\mathrm{Fu}$ [41] also have given a direct uniqueness proof (free from the Stroh formalism) for this problem.

Once the existence has been established, the surface-wave velocity must be determined from implicit or explicit secular equations. A number of approaches have been suggested based on the Stroh formalism (see, for example, Barnett \& Lothe [37]; Chadwick \& Wilson [42]) that 
evaluate the surface-wave velocity from implicit secular equations. Although all of these approaches are straight-forward, their use requires familiarity with the Stroh formalism and a considerable amount of numerical work. Another approach, based on the surface-impedance matrix, has been proposed recently by Fu and Mielke [40]. This method is very practical and efficient. Nevertheless, one can also find the surface-wave velocity by directly solving explicit secular equations. A large number of such secular equations have been derived by employing different methods such as the polarization vector method [43; 44], the method of first integrals [45; 46] and the co-factor method [47]. These methods were concisely presented by Ting in [48]. The explicit secular equations obtained by the methods just mentioned often admit spurious roots that have to be carefully eliminated, as opposed to the numerical methods based on the Stroh formulation or on the surface-impedance matrix. However, the application of explicit secular equations is not limited to numerically determine the surface-wave velocity. They are also convenient tools to solve the inverse problem that deals with measured values of the wave speed and their agreement with material parameters as well as other practical applications. Such expressions have been given by Vinh \& Ogden [49], Malischewsky [50] for isotropic solids, Ogden \& Vinh [51], Vinh \& Ogden [52; 53] for orthotropic solids and Vinh [54; 55] for pre-stressed media, among others. These investigations deal with harmonic surface waves travelling along the traction-free flat surface of half-spaces. Surface waves with arbitrary profile as well as surface waves guided by topography and those traveling along forced surfaces have also attracted attention and we refer to Kiselev and Rogerson [56], Kiselev and Parker [57], Prikazchikov [58], Parker [59], Adams et al. [32], Fu et al. [60], Kaplunov et al. [61] and references therein.

This thesis presents the development of implicit and explicit secular equations corresponding to Rayleigh waves in nonlinear elastic media considering different scenarios representative of important current problems in engineering and biomechanics.

In chapter two of this thesis, we use the polarization vector method to obtain the explicit secular equation of non-principal Rayleigh waves propagating in incompressible, transversely isotropic, pre-stressed elastic 
half-spaces.

In chapter three, we extend the analysis presented in chapter 2 [62] dealing with Rayleigh waves for materials reinforced with one family of fibers to obtain the explicit and implicit secular equations of non-principal Rayleigh waves propagating in imcompressible materials reinforced with two families of fibers in the framework of nonlinear elasticity [63].

In chapter four, we obtain the explicit secular equation of surface waves in the situation of a layered half-space subject to finite deformation and a pre-stress associated with it through a constitutive law, for materials with an initial stress parallel to the half-space surface [64]. The layer is taken to have a uniform finite thickness and material properties different from those of the half-space, and the initial stress is assumed to be different in the layer and half-space. In the presence of the initial stress (in the reference configuration) the strain-energy function depends on the initial stress as well as on the deformation from the reference configuration.

In chapters 2, 3 and 4 we confirm the quality of the analytical results with numerical results and derive interesting conclusions.

Finally, in chapter five we summarize the most important conclusions obtained in this thesis. 


\section{Rayleigh waves in incompressible materials reinforced with one fiber

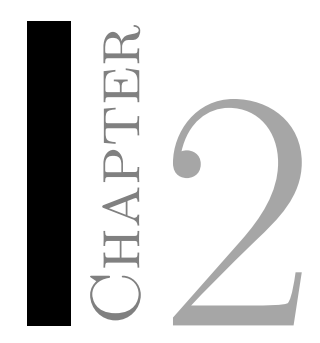

\section{$2.1 \quad$ Introduction}

In this chapter we obtain the explicit secular equation of non-principal Rayleigh waves propagating in incompressible, transversely isotropic, prestressed elastic half-spaces. In order to obtain such equation, one can apply either the polarization vector method or the co-factor method since the method of first integrals is not applicable in this case, see Ting [48]. We choose the polarization vector method, which seems to be simpler. The propagation of Rayleigh under the conditions at hand was investigated by Prikazchikov and Rogerson [65] who derived the secular equation in implicit form.

The motivation behind this analysis can be summarized as follows. Firstly, the explicit secular equation is a convenient tool for (among others) the non-destructive evaluation of prestressed structures before and during loading, see for example, Makhort [66; 67]; Hirao et al.[68]; Husson [69]; Delsanto and Clark [70]; Dyquennoy et al. [71; 72]; Hu et al. [73]. Secondly, the use of fibre-reinforced elastic composites is common in engineering applications because these materials have a higher strength-to-weight ratio than classical isotropic materials used in the past. Fibre-reinforced elastic composites with a family of parallel fibres reinforcing a material are called transversely isotropic elastic materials. In addition, the acoustics of incompressible soft solids is being used in the analysis of biological soft tissues, see for instance Destrade et al. [74] 
as well as [75] and [76]. Soft biological tissues were generally considered incompressible and isotropic during the early days of their analyses. In more recent years some have been recognized as highly anisotropic due to the presence of collagen fibers [77; 78]. The mechanical modeling of these materials is related to the analysis of Rayleigh waves propagating in transversely isotropic, incompressible, pre-stressed elastic half-spaces.

The chapter is organized as follows. In Section 2.2, the basic constitutive equations for the hyperelastic material model at hand are given. Similarly, the corresponding equations for infinitesimal waves superimposed on a finite deformation consisting of a pure homogeneous strain are outlined. We introduce the equations of motion in matrix form. Surface waves are studied in Section 2.3. The Stroh formalism is derived in Subsection 2.3.1. The implicit secular equation is presented in Subsection 2.3.2. Then, it will be used to eliminate spurious roots arising in the explicit secular equation. The secular equation for non-principal Rayleigh waves in incompressible, transversely isotropic, pre-stressed elastic half-spaces is obtained. The results are illustrated in respect of the so-called reinforcing models and exact formulas for the Rayleigh wave speed are given for two special cases in which the propagation direction of the wave and the fiber reinforcement direction are restricted to be a principal direction of the primary strain.

\subsection{Material model and equations of motion}

\subsubsection{Material model}

First, we introduce the material model. For that purpose, it is sufficient to consider an elastic body whose initial geometry defines a reference configuration, which we denote by $\mathcal{B}_{0}$ and a finitely deformed equilibrium configuration $\mathcal{B}_{t}$. The deformation gradient tensor associated with the deformation $\mathcal{B}_{0} \rightarrow \mathcal{B}_{t}$ is denoted by $\mathbf{F}$.

In the literature, several composite materials (see [79] and references therein) and also some soft tissues are modeled as incompressible 
transversely isotropic elastic solid with one preferred direction associated with a family of parallel fibers of collagen. We denote by $\mathbf{M}$ the unit vector in that direction when the solid is unloaded and at rest.

The most general transversely isotropic nonlinear elastic strain-energy function $\Omega$ depends on $\mathbf{F}$ through the invariants associated with $\mathbf{M}$ and the right Cauchy-Green strain tensor $\mathbf{C}=\mathbf{F}^{\mathrm{T}} \mathbf{F}$ (see, for instance, [80]). The invariants of $\mathbf{C}$ most commonly used are the principal invariants, defined by

$$
I_{1}=\operatorname{tr} \mathbf{C}, \quad I_{2}=\frac{1}{2}\left[(t r \mathbf{C})^{2}-\operatorname{tr}\left(\mathbf{C}^{2}\right)\right], \quad I_{3}=\operatorname{det} \mathbf{C} .
$$

The (anisotropic) invariants associated with $\mathbf{M}$ and $\mathbf{C}$ are usually taken as

$$
I_{4}=\mathbf{M} \cdot(\mathbf{C M}), \quad I_{5}=\mathbf{M} \cdot\left(\mathbf{C}^{2} \mathbf{M}\right)
$$

It follows that for incompressible materials $\Omega=\Omega\left(I_{1}, I_{2}, I_{4}, I_{5}\right)$ since $I_{3}=1$ at all times.

For the considered incompressible material the Cauchy stress is

$$
\boldsymbol{\sigma}=\mathbf{F} \frac{\partial \Omega}{\partial \mathbf{F}}-p \mathbf{I}=\sum_{i=1, i \neq 3}^{5} \Omega_{i} \mathbf{F} \frac{\partial I_{i}}{\partial \mathbf{F}}-p \mathbf{I}
$$

where $p$ is a Lagrange multiplier associated with the incompressibility constraint and $\mathbf{I}$ is the $3 \times 3$ identity matrix. The principal values are denoted by $\sigma_{i}, i=1,2,3$. The Cauchy stress tensor can be written as

$$
\begin{aligned}
\boldsymbol{\sigma}= & 2 \Omega_{1} \mathbf{B}+2 \Omega_{2}\left(I_{1} \mathbf{I}-\mathbf{B}\right) \mathbf{B}+2 \Omega_{4} \mathbf{m} \otimes \mathbf{m} \\
& +2 \Omega_{5}(\mathbf{m} \otimes \mathbf{B m}+\mathbf{B m} \otimes \mathbf{a})-p \mathbf{I},
\end{aligned}
$$

where $\mathbf{m}=\mathbf{F M}$. Hence, $\mathbf{m}$ gives the fiber direction in the deformed configuration. It is clear that principal directions of stress and strain do not coincide, in general.

In the biomechanics literature several strain energy functions given by an isotropic elastic material augmented with the so-called reinforcing model 
can be found. It is common to consider functions of the form (see [79])

$$
\Omega=\mu\left(I_{1}-3\right) / 2+f\left(I_{4}\right),
$$

where $f\left(I_{4}\right)$ is the reinforcing model. There are other reinforcing models (see [81]), in particular, some strain energy functions also have the form

$$
\Omega=\mu\left(I_{1}-3\right) / 2+g\left(I_{5}\right) .
$$

We will make use of these reinforcing models to illustrate the results.

\subsubsection{Linearized equations of motion: Incremental equations}

We introduce now the precise notation that is convenient for the analysis developed in the next sections. Let $\left(X_{1}, X_{2}, X_{3}\right)$ be a fixed rectangular coordinate system. Consider an incompressible transversely isotropic semiinfinite body $\mathcal{B}$ in its unstrained state $\mathcal{B}_{0}$ that occupies the region $X_{2} \geq 0$. Fibers run parallel to each other and perpendicular to the depth direction $X_{2}$, i.e. $M_{2}=0$. The body is subjected to a finite pure homogeneous strain with principal directions given by the $X_{i}$-axes. A finitely deformed (prestressed) equilibrium state $\mathcal{B}_{e}$ is obtained. A small time-dependent motion is superimposed upon this pre-stressed equilibrium configuration to reach a final material state $\mathcal{B}_{t}$, called current configuration. The position vectors of a representative particle are denoted by $X_{i}, x_{i}(\mathbf{X}), \tilde{x}_{i}(\mathbf{X}, t)$ in $\mathcal{B}_{0}, \mathcal{B}_{e}$ and $\mathcal{B}_{t}$, respectively (see Figure 2.1). The deformation gradient tensor associated with the deformations $\mathcal{B}_{0} \rightarrow \mathcal{B}_{t}$ and $\mathcal{B}_{0} \rightarrow \mathcal{B}_{e}$ are denoted by $\mathbf{F}$ and $\overline{\mathbf{F}}$ (which is homogeneous), respectively, and are given in component form by

$$
F_{i A}=\frac{\partial \tilde{x}_{i}}{\partial X_{A}}, \bar{F}_{i A}=\frac{\partial x_{i}}{\partial X_{A}}
$$

It is clear from (2.7) that

$$
F_{i A}=\left(\delta_{i j}+u_{i, j}\right) \bar{F}_{j A}
$$

where $\delta_{i j}$ is the Kronecker operator, $u_{i}(\mathbf{X}, t)$ denotes the small time-dependent displacement associated with the deformation $\mathcal{B}_{e} \rightarrow \mathcal{B}_{t}$ 
and a comma indicates differentiation with respect to the indicated spatial coordinate in $\mathcal{B}_{e}$. The fibre orientation in $\mathcal{B}_{e}$ is in particular related to $\mathbf{M}$ by $\mathbf{m}=\overline{\mathbf{F}} \mathbf{M}$.

The necessary equations including the linearized equations of motion for transversely isotropic incompressible materials are summarized below. We write the equations of motion in matrix form. For that purpose we introduce first the elasticity tensor.

The incremental components of the nominal stress tensor $S_{j i}$ are related to the incremental displacement gradients $u_{k, l}$ by (see [82])

$$
S_{j i}=\mathcal{A}_{j i l k} u_{k, l}+P u_{j, i}-p^{*} \delta_{i j}
$$

where $P=p(\overline{\mathbf{F}})$ is the value of $p$ at $\mathcal{B}_{e}$ (independent of time $t$ ), $p^{*}=p-P$ is the time-dependent increment of $p$ and the components of the (pushed forward ) fourth-order elasticity tensor $\mathbf{A}$ for $\Omega=\Omega\left(I_{1}, I_{2}, I_{4}, I_{5}\right)$ are given by (see also formula A7 in [75] or in [83] )

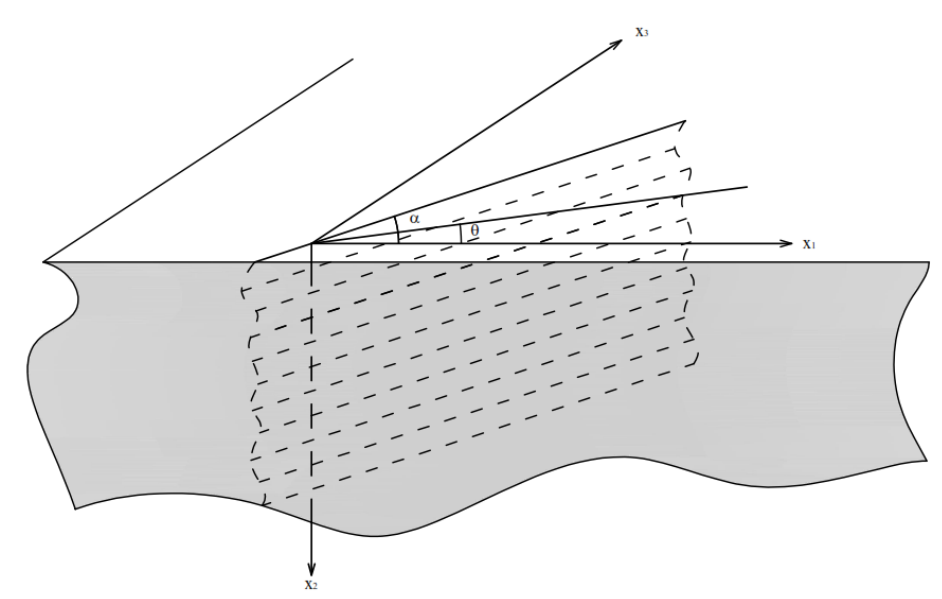

Figure 2.1: A surface wave propagates in a principal plane of the pre-strain, the plane $x_{2}=0$, but not in a principal direction, its propagation direction makes an angle $\theta$ with the $x_{1}$-axis. The material is reinforced by parallel fibers which make an angle $\alpha$ with the $x_{1}$-axis.

In the absence of body forces, incremental equations of motion and incremental version of the incompressiblility are (see [82])

$$
S_{i j, i}=\rho \ddot{u}_{j}, i, j=1,2,3,
$$




$$
u_{k, k}=0
$$

where a dot indicates differentiation with respect to time $t$.

From Eqs. (3.10), (3.16) and (3.17) we have:

$$
\eta^{\prime}=\left[\begin{array}{ll}
\mathrm{Q}_{1} & \mathrm{Q}_{2} \\
\mathrm{Q}_{3} & \mathrm{Q}_{1}^{T}
\end{array}\right] \eta
$$

within which the prime denotes differentiation with respect to $x_{2}$, $\eta=\left[\begin{array}{llllll}u_{1} & u_{2} & u_{3} & S_{21} & S_{22} & S_{23}\end{array}\right]^{T}$ and:

$$
\mathbf{Q}_{1}=\left[\begin{array}{ccc}
0 & a_{11} \partial_{1}+a_{13} \partial_{3} & 0 \\
-\partial_{1} & 0 & -\partial_{3} \\
0 & a_{31} \partial_{1}+a_{33} \partial_{3} & 0
\end{array}\right], \quad \mathbf{Q}_{2}=\left[\begin{array}{ccc}
d_{1} & 0 & -d_{13} \\
0 & 0 & 0 \\
-d_{13} & 0 & d_{3}
\end{array}\right]
$$

$\mathbf{Q}_{3}=\left[\begin{array}{ccc}\rho \partial_{t}^{2}-b_{111} \partial_{1}^{2}- & 0 & -b_{311} \partial_{1}^{2}-b_{313} \partial_{13}^{2} \\ b_{113} \partial_{13}^{2}-b_{133} \partial_{3}^{2} & & -b_{333} \partial_{3}^{2} \\ 0 & \rho \partial_{t}^{2}-e_{11} \partial_{1}^{2}-e_{13} \partial_{13}^{2}-e_{33} \partial_{3}^{2} & 0 \\ -b_{311} \partial_{1}^{2}-b_{313} \partial_{13}^{2} & 0 & \rho \partial_{t}^{2}-d_{311} \partial_{1}^{2} \\ -b_{333} \partial_{3}^{2} & 0 & -d_{313} \partial_{13}^{2}-d_{333} \partial_{3}^{2}\end{array}\right]$ 
in which

$$
\begin{aligned}
a_{11} & =\left(\mathcal{A}_{2123} \mathcal{A}_{1223}-\mathcal{A}_{2323} \mathcal{A}_{1221}^{*}\right) / d, a_{13}=\left(\mathcal{A}_{2123} \mathcal{A}_{2332}^{*}-\mathcal{A}_{2323} \mathcal{A}_{2132}\right) / d, \\
a_{31} & =\left(\mathcal{A}_{2123} \mathcal{A}_{1221}^{*}-\mathcal{A}_{2121} \mathcal{A}_{2312}\right) / d, a_{33}=\left(\mathcal{A}_{2123} \mathcal{A}_{2132}-\mathcal{A}_{2121} \mathcal{A}_{2332}^{*}\right) / d, \\
d & =\mathcal{A}_{2121} \mathcal{A}_{2323}-\mathcal{A}_{2123}^{2}, d_{1}=\mathcal{A}_{2323} / d, d_{3}=\mathcal{A}_{2121} / d, d_{13}=\mathcal{A}_{2123} / d, \\
b_{111} & =\left(\mathcal{A}_{1111}^{*}+\mathcal{A}_{2222}^{*}-2 \mathcal{A}_{1122}\right), b_{113}=2\left(\mathcal{A}_{1131}-\mathcal{A}_{2231}\right), b_{133}=\mathcal{A}_{3131}, \\
b_{311} & =\left(\mathcal{A}_{1113}-\mathcal{A}_{2213}\right), b_{313}=\left(\mathcal{A}_{2222}^{*}+\mathcal{A}_{1331}^{*}+\mathcal{A}_{1133}-\mathcal{A}_{1122}-\mathcal{A}_{3322}\right), \\
b_{333} & =\left(\mathcal{A}_{3133}-\mathcal{A}_{3122}\right), \\
d_{311} & =\mathcal{A}_{1313}, d_{313}=2\left(\mathcal{A}_{1333}-\mathcal{A}_{1322}\right), d_{333}=\left(\mathcal{A}_{3333}^{*}+\mathcal{A}_{2222}^{*}-2 \mathcal{A}_{2233}\right), \\
e_{11} & =\left(\mathcal{A}_{1212}+a_{11} \mathcal{A}_{1221}^{*}+a_{31} \mathcal{A}_{1223}\right), e_{13}=\left(2 \mathcal{A}_{1232}+a_{11} \mathcal{A}_{3221}+a_{13} \mathcal{A}_{1221}^{*}\right. \\
& \left.+a_{31} \mathcal{A}_{3223}^{*}+a_{33} \mathcal{A}_{1223}\right), e_{33}=\left(\mathcal{A}_{3232}+a_{13} \mathcal{A}_{3221}+a_{33} \mathcal{A}_{2332}^{*}\right) .
\end{aligned}
$$

Here the notations $\partial_{k}=\partial / \partial x_{k}, \partial_{k}^{2}=\partial^{2} / \partial x_{k}^{2}, \partial_{i j}^{2}=\partial^{2} / \partial x_{i} \partial x_{j}, \partial_{t}^{2}=$ $\partial^{2} / \partial t^{2}$ and $\mathcal{A}_{p i q j}^{*}=\mathcal{A}_{p i q j}+P$ have been used. It is clear that $\mathbf{Q}_{2}=\mathbf{Q}_{2}^{T}$ and $\mathbf{Q}_{3}=\mathbf{Q}_{3}^{T}$. Equation (2.12) in which matrices $\mathbf{Q}_{k}$ are given by (2.13) and (2.14) is called the incremental equation in matrix form.

\section{$2.3 \quad$ Surface waves}

The analysis is specialized to Rayleigh waves propagating in a principal plane of the pre-strain, the plane $x_{2}=0$, but not in a principal direction. The incremental equation of motion in matrix form can be cast as a homogeneous linear system of six first-order differential equations.

\subsubsection{The Stroh formulation}

We consider a Rayleigh wave traveling with velocity $c$ and with its wave vector $\mathbf{k}$ laying in the $\left(x_{1}, x_{3}\right)$ plane. The wave makes an angle $\theta$ with the $x_{1}$-direction and decays in the $x_{2}$-direction. Then, the displacements and stresses of the Rayleigh wave are written (see [84]) as

$$
u_{n}=U_{n}(y) e^{i k\left(x_{1} c_{\theta}+x_{3} s_{\theta}-c t\right)}, S_{2 n}=i k t_{n}(y) e^{i k\left(x_{1} c_{\theta}+x_{3} s_{\theta}-c t\right)}, n=1,2,3, y=k x_{2}
$$


respectively, where $c_{\theta}=\cos \theta, s_{\theta}=\sin \theta$, and $k=|\mathbf{k}|$ is the wave number. Substituting (3.18) into Eq. (2.12) leads to

$$
\xi^{\prime}=i \mathbf{N} \xi, 0 \leq y<+\infty
$$

where the prime signifies differentiation with respect to $y$ and

$$
\xi=\left[\begin{array}{c}
\mathbf{u} \\
\mathbf{t}
\end{array}\right], \mathbf{u}=\left[\begin{array}{c}
U_{1} \\
U_{2} \\
U_{3}
\end{array}\right], \mathbf{t}=\left[\begin{array}{c}
t_{1} \\
t_{2} \\
t_{3}
\end{array}\right], \mathbf{N}=\left[\begin{array}{cc}
\mathbf{N}_{1} & \mathbf{N}_{2} \\
\mathbf{K} & \mathbf{N}_{1}^{T}
\end{array}\right]
$$

within which the matrices $\mathbf{N}_{k}$ and $\mathbf{K}$ are defined by

$$
\mathbf{N}_{1}=\left[\begin{array}{ccc}
0 & f_{1} & 0 \\
-c_{\theta} & 0 & -s_{\theta} \\
0 & f_{2} & 0
\end{array}\right], \mathbf{N}_{2}=\left[\begin{array}{ccc}
d_{1} & 0 & -d_{13} \\
0 & 0 & 0 \\
-d_{13} & 0 & d_{3}
\end{array}\right], \mathbf{K}=\left[\begin{array}{ccc}
h_{1} & 0 & h_{2} \\
0 & h_{3} & 0 \\
h_{2} & 0 & h_{4}
\end{array}\right]
$$

where:

$$
\begin{aligned}
& f_{1}=a_{11} c_{\theta}+a_{13} s_{\theta}, \quad f_{2}=a_{31} c_{\theta}+a_{33} s_{\theta}, \\
& h_{1}=\rho c^{2}-b_{111} c_{\theta}^{2}-b_{113} c_{\theta} s_{\theta}-b_{133} s_{\theta}^{2}, \quad h_{2}=-b_{311} c_{\theta}^{2}-b_{313} c_{\theta} s_{\theta}-b_{333} s_{\theta}^{2}, \\
& h_{3}=\rho c^{2}-e_{11} c_{\theta}^{2}-e_{13} c_{\theta} s_{\theta}-e_{33} s_{\theta}^{2}, \quad h_{4}=\rho c^{2}-d_{311} c_{\theta}^{2}-d_{313} c_{\theta} s_{\theta}-d_{333} s_{\theta}^{2} .
\end{aligned}
$$

Equation (3.19) is the Stroh formulation [33; 34]. The decay condition is expressed in the following form

$$
\xi(+\infty)=\mathbf{0}
$$

The boundary condition of zero incremental traction using the expression given for $S_{2 n}$ in (3.18) means that

$$
\mathbf{t}(0)=\mathbf{0} \text {. }
$$

\subsubsection{Implicit and explicit secular equation}

The secular equation in implicit form reads

$$
n \omega_{I}-\left(m-\omega_{I I}\right) \omega_{I I I}=0
$$


as show in $[43 ; 84]$, where $m, n$

$$
\begin{aligned}
m & =\left(\frac{f_{1}^{2}}{h_{3}}-d_{1}\right) h_{1}+\left(\frac{f_{2}^{2}}{h_{3}}-d_{3}\right) h_{4}+2\left(\frac{f_{1} f_{2}}{h_{3}}+d_{13}\right) h_{2} \\
n & =\left(h_{1} h_{4}-h_{2}^{2}\right)\left[d_{1} d_{3}-\frac{d_{3} f_{1}^{2}+d_{1} f_{2}^{2}}{h_{3}}-\frac{2 d_{13} f_{1} f_{2}}{h_{3}}-d_{13}^{2}\right] .
\end{aligned}
$$

Here,

$$
\omega_{I}=-\left(s_{1}+s_{2}+s_{3}\right), \omega_{I I}=s_{1} s_{2}+s_{2} s_{3}+s_{3} s_{1}, \omega_{I I I}=-s_{1} s_{2} s_{3},
$$

where $s_{i}$ are the eigenvalues of the Stroh matrix $\mathbf{N}$ with positive imaginary part.

Equation (2.23) is called the implicit secular equation (see also Destrade et al. [84]) because the expressions for the $\omega_{I}, \omega_{I I}$ and $\omega_{I I I}$ in terms of $X$ are not known. Using (3.24) one obtains

$$
\overline{\mathbf{u}}^{T}(0) \mathbf{K}^{(n)} \mathbf{u}(0)=0 \forall n \in \mathbf{Z}
$$

Let us consider that $U_{1}(0) \neq 0$, then the vector $\mathbf{u}(0)$ can be written as $\mathbf{u}(0)=U_{1}(0)\left[\begin{array}{lll}1 & \alpha & \beta\end{array}\right]^{T}$, where $\alpha=U_{2}(0) / U_{1}(0)$ and $\beta=U_{3}(0) / U_{1}(0)$ are complex numbers and $\alpha=\alpha_{1}+i \alpha_{2}$ and $\beta=\beta_{1}+i \beta_{2}$, where $\alpha_{1}, \alpha_{2}, \beta_{1}, \beta_{2}$ are real. We notice that $[1, \alpha, \beta]^{T}$ is the so called polarization vector $\mathbf{a}_{R}^{T}$ (see [44]). Introducing the expression of $\mathbf{u}(0)$ into (3.28) lead to

$$
\begin{aligned}
K_{11}^{(n)}+K_{22}^{(n)}\left(\alpha_{1}^{2}+\alpha_{2}^{2}\right)+K_{33}^{(n)}\left(\beta_{1}^{2}+\beta_{2}^{2}\right) & +2 K_{12}^{(n)} \alpha_{1}+2 K_{13}^{(n)} \beta_{1} \\
& +2 K_{23}^{(n)}\left(\alpha_{1} \beta_{1}+\alpha_{2} \beta_{2}\right)=0 .
\end{aligned}
$$

Equation (2.27) for $n=-1,1,3$ can be written as

$$
\mathbf{K}_{2} \alpha_{2}^{2}+\mathbf{K}_{3} \beta_{1}^{2}+\mathbf{K}_{4}\left(2 \beta_{1}\right)=-\mathbf{K}_{1}
$$




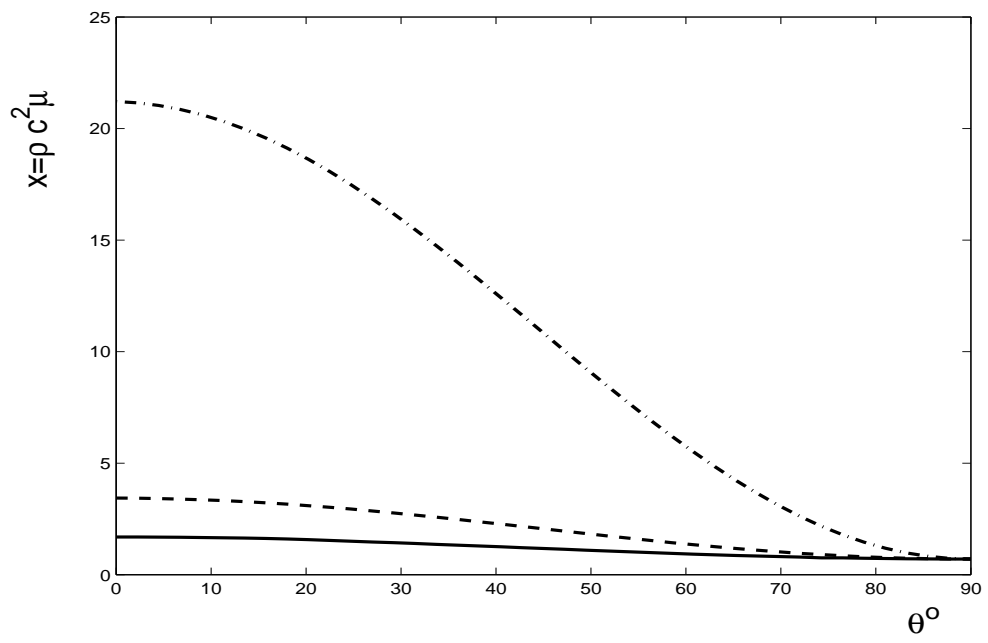

Figure 2.2: The curves show the dependence of $x=\rho c^{2} / \mu$ obtained using (3.30) on $\theta \in[0 \pi / 2]$, which is the angle that the propagating wave makes with the $x_{1}$-direction. Fibers are parallel to the $X_{1}$-direction, the elastic half-space is initially under uniaxial tension along the $X_{1}$-axis and the strain-energy function is given by (2.33) (dashed line), (2.34) (dash-dot line) and (2.35) (solid line) for $\gamma_{1}=\gamma_{2}=3 / 4$ and $\lambda=1.3$.

where

$$
\mathbf{K}_{1}=\left[\begin{array}{c}
K_{11}^{(-1)} \\
K_{11}^{(1)} \\
K_{11}^{(3)}
\end{array}\right], \quad \mathbf{K}_{2}=\left[\begin{array}{c}
K_{22}^{(-1)} \\
K_{22}^{(1)} \\
K_{22}^{(3)}
\end{array}\right], \quad \mathbf{K}_{3}=\left[\begin{array}{c}
K_{33}^{(-1)} \\
K_{33}^{(1)} \\
K_{33}^{(3)}
\end{array}\right], \quad \mathbf{K}_{4}=\left[\begin{array}{c}
K_{13}^{(-1)} \\
K_{13}^{(1)} \\
K_{13}^{(3)}
\end{array}\right]
$$

in which

$$
\begin{aligned}
K_{11}^{(1)} & =h_{1}, \quad K_{13}^{(1)}=h_{2}, \quad K_{22}^{(1)}=h_{3}, \quad K_{33}^{(1)}=h_{4}, \\
K_{11}^{(3)}= & d_{1} h_{1}^{2}+d_{3} h_{2}^{2}-2 d_{13} h_{1} h_{2}-2\left(f_{1} h_{1}+f_{2} h_{2}\right) c_{\theta}+h_{3} c_{\theta}^{2}, \\
K_{13}^{(3)}= & d_{1} h_{1} h_{2}-d_{13}\left(h_{2}^{2}+h_{1} h_{4}\right)+d_{3} h_{2} h_{4} \\
& -\left(f_{1} h_{1}+f_{2} h_{2}\right) s_{\theta}-\left(f_{1} h_{2}+f_{2} h_{4}\right) c_{\theta}+h_{3} s_{\theta} c_{\theta}, \\
K_{22}^{(3)}= & f_{1}^{2} h_{1}+2 f_{1} f_{2} h_{2}-2 f_{1} h_{3} c_{\theta}+f_{2}^{2} h_{4}-2 f_{2} h_{3} s_{\theta}, \\
K_{33}^{(3)}= & d_{1} h_{2}^{2}+d_{3} h_{4}^{2}-2 d_{13} h_{2} h_{4}-2\left(f_{1} h_{2}+f_{2} h_{4}\right) s_{\theta}+h_{3} s_{\theta}^{2}, \\
K_{11}^{(-1)}= & \left(d_{3} h_{3}-f_{2}^{2}\right)\left(h_{4} c_{\theta}^{2}-2 h_{2} c_{\theta} s_{\theta}+h_{1} s_{\theta}^{2}\right), \\
K_{13}^{(-1)}= & \left(d_{13} h_{3}+f_{1} f_{2}\right)\left(h_{4} c_{\theta}^{2}-2 h_{2} c_{\theta} s_{\theta}+h_{1} s_{\theta}^{2}\right), \\
K_{22}^{(-1)}= & {\left[2 d_{13} f_{1} f_{2}+d_{1} f_{2}^{2}+d_{13}^{2} h_{3}+d_{3}\left(f_{1}^{2}-d_{1} h_{3}\right)\right]\left(h_{1} h_{4}-h_{2}^{2}\right), } \\
K_{33}^{(-1)} & =\left(d_{1} h_{3}-f_{1}^{2}\right)\left(h_{4} c_{\theta}^{2}-2 h_{2} c_{\theta} s_{\theta}+h_{1} s_{\theta}^{2}\right)
\end{aligned}
$$


Note that the elements $K_{i j}^{(-1)}$ are determined up to a factor $1 / \operatorname{det} \mathbf{N}$.

Using (2.28) one obtains that

$$
\alpha_{2}^{2}=-\frac{\left|\mathbf{K}_{1}, \mathbf{K}_{3}, \mathbf{K}_{4}\right|}{\left|\mathbf{K}_{2}, \mathbf{K}_{3}, \mathbf{K}_{4}\right|}, \quad \beta_{1}=\frac{-\left|\mathbf{K}_{2}, \mathbf{K}_{3}, \mathbf{K}_{1}\right|}{2\left|\mathbf{K}_{2}, \mathbf{K}_{3}, \mathbf{K}_{4}\right|}, \quad \beta_{1}^{2}=-\frac{\left|\mathbf{K}_{2}, \mathbf{K}_{1}, \mathbf{K}_{4}\right|}{\left|\mathbf{K}_{2}, \mathbf{K}_{3}, \mathbf{K}_{4}\right|} .
$$

Using $(2.31)_{2}$ and $(2.31)_{3}$ the secular equation in explicit form reads

$$
\left|\mathbf{K}_{2}, \mathbf{K}_{3}, \mathbf{K}_{1}\right|^{2}+4\left|\mathbf{K}_{2}, \mathbf{K}_{3}, \mathbf{K}_{4}\right|\left|\mathbf{K}_{2}, \mathbf{K}_{1}, \mathbf{K}_{4}\right|=0
$$

Therefore Eq. (3.30) is an algebraic equation of order 12 in $X$. The numerical resolution of (3.30) yields a priori 12 roots for $X$. From these, it is easy to discard the complex roots, the negative real roots, and the roots corresponding to supersonic surface waves. For the remaining roots, we use (2.23) to eliminate the ones that do not correspond to a subsonic Rayleigh wave. There is only one (subsonic) Rayleigh wave (see Fu [85]). In order to illustrate the results further we consider some particular strainenergy functions. Numerical resolution of the polynomial (3.30) yields the wave velocity. The expressions are quite lengthy and not enlightening, so we omit them and just provide some numerical results. We consider the following strain-energy functions (see, for instance, [79; 81])

$$
\begin{gathered}
\Omega^{1}=\frac{\mu}{2}\left(I_{1}-3\right)+\frac{\mu \gamma_{1}}{2}\left(I_{4}-1\right)^{2}, \\
\Omega^{2}=\frac{\mu}{2}\left(I_{1}-3\right)+\frac{\mu \gamma_{2}}{2}\left(I_{5}-1\right)^{2}, \\
\Omega^{3}=\frac{\mu}{2}\left(I_{1}-3\right),
\end{gathered}
$$

where $\mu, \gamma_{1}$ and $\gamma_{2}$ are material constants. The last model is the well known neo-Hookean one. The other two strain energy functions introduce reinforcing models. First, for simplicity, we consider that the fibers are parallel to the $X_{1}$-direction and that the elastic half-space is initially under uniaxial tension along the $X_{1}$-axis (see $[75 ; 76]$ ), i. e.

$$
x_{1}=\lambda X_{1}, x_{2}=\lambda^{-1 / 2} X_{2}, x_{3}=\lambda^{-1 / 2} X_{3}, \lambda>0, \lambda=\text { const. }
$$

The wave makes an angle $\theta$ with the $x_{1}$-direction. Figure 2.2 shows the 


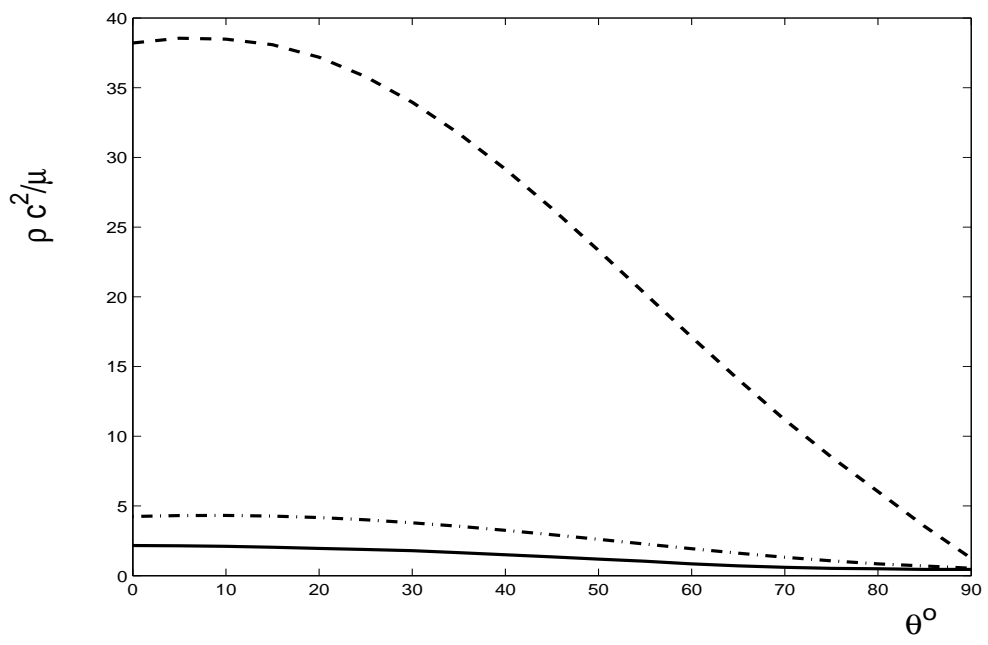

Figure 2.3: Values of $x=\rho c^{2} / \mu$ obtained using (3.30) vs $\theta \in[0 \pi / 2]$ when the fibre direction makes an angle $\alpha=\pi / 6$ with the $x_{1}$-axis for (2.33) (dashdot line), (2.34) (dashed line) and (2.35) (solid line), with $\gamma_{1}=\gamma_{2}=3 / 4$ and $\left(\lambda_{1}, \lambda_{2}, \lambda_{3}\right)=(3 / 2,1,2 / 3)$.

dependence of the squared dimensionless Rayleigh wave velocity $x=\rho c^{2} / \mu$ obtained using (3.30) on $\theta$. The anisotropy strongly influences the Rayleigh wave velocity of the isotropic base model. Furthermore, the influence on the wave velocity of the isotropic base model introduced by the invariant $I_{5}$ is stronger than the one given by the invariant $I_{4}$. As it is shown in Figure 2.2 , if the surface wave propagates in a direction perpendicular to the fiber direction then the wave velocity is associated with the one corresponding to the neo-Hookean material without reinforcement as given by Flavin [86]. Let us consider now that the fiber direction is not a principal direction. The pre-strain is pure homogeneous and given by

$$
x_{1}=\lambda_{1} X_{1}, x_{2}=\lambda_{2} X_{2}, x_{3}=\lambda_{3} X_{3},
$$

where $\lambda_{k}$ are the principal stretches of the deformation and obey that $\lambda_{1} \lambda_{2} \lambda_{3}=1$. We further consider that the plane $x_{2}=0$ is free of tractions. Under these conditions, the components of the Cauchy stress are obtained, after some simple manipulations, using (2.4) for a given strain energy function.

Figure 2.3 shows the dependence on $\theta \in[0 \pi / 2]$ (the angle between the wave propagation direction and the $x_{1}$-axis) of $x=\rho c^{2} / \mu$ obtained using 
(3.30) when the fiber direction makes an angle $\alpha=\pi / 6$ with the $x_{1}$-axis for (2.33) (dash-dot line), (2.34) (dashed line) and (2.35) (solid line). The parameters used for the computations are $\gamma_{1}=\gamma_{2}=3 / 4$, while $\lambda_{1}=3 / 2$, $\lambda_{2}=1$ and $\lambda_{3}=2 / 3$. The curve associated with the neo-hookean model has a maximum for $\theta=0$. On the other hand, the other two curves have a maximum at an angle $\theta \in(0 \pi / 2)$.

Finally, we check that when Equation (3.30) is specialized to isotropic materials (using the results of section 3.1.1) coincides with Eq. (4.5) in [84], which is the secular equation for non-principal Rayleigh waves in deformed isotropic incompressible materials.

In what follows we analyze for a general transversely isotropic incompressible material two special cases where the propagation direction of the wave and the fiber reinforcement direction are restricted to be a principal direction of the primary strain. 
RAYLEIGH WAVES IN INCOMPRESSIBLE MATERIALS REINFORCED WITH ONE FIBER 


\section{Rayleigh waves in incompressible materials reinforced with two fibers}

\subsection{Introduction}

In this chapter we extend the analysis presented in chapter 2 [62] dealing with Rayleigh waves for materials reinforced with one family of fibers to materials reinforced with two families of fibers in the framework of nonlinear elasticity. This is motivated by several factors. First, the use of doubly fiber-reinforced elastic composites is common in engineering applications. In addition, there is a lot of interest in the acoustics of biological soft tissues (see. for example, Destrade et al. [74]). Soft biological tissues have been recognized as highly anisotropic due to the presence of collagen fibers [77] and are modeled as orthotropic materials with two families of fibers.

We establish a procedure to obtain both the explicit and implicit secular equations of non-principal Rayleigh waves propagating in incompressible, doubly fiber-reinforced, pre-stressed elastic half-spaces. For transversely isotropic materials the explicit secular equation was given in [62] while the implicit one was given in [65]. We build upon these results and use the polarization vector method to get the secular equation in explicit form. The implicit secular equation is obtained from the so-called propagation condition. The latter equation is used to eliminate the spurious roots that arise in the explicit secular equation.

In Section 3.2, the basic constitutive equations associated with this study are presented. This includes the material model as well as the 
corresponding equations for infinitesimal waves superimposed on a finite deformation consisting of a pure homogeneous strain. In Section 3.3, the Stroh formalism is applied to the analysis of infinitesimal surface waves propagating in a statically, finitely and homogeneously deformed doubly fiber-reinforced half-space. The free surface is assumed to coincide with one of the principal planes of the primary strain, but a propagating surface wave is not restricted to a principal direction (see [87] for a parallel work that enlightens this analysis). The implicit and explicit secular equations are presented. In Section 3.4, the results are illustrated numerically in respect of a strain-energy function used to model soft tissue (see [77]).

\subsection{Basic equations}

\subsubsection{Kinematics}

Consider an elastic body whose reference configuration is denoted by $\mathcal{B}_{0}$ and a finitely deformed equilibrium configuration. The deformation gradient tensor associated with the deformation is denoted by $\mathbf{F}$. In addition, let $\left(X_{1}, X_{2}, X_{3}\right)$ be a fixed rectangular coordinate system in $\mathcal{B}_{0}$. The precise notation necessary for the analysis will be introduced later on.

Composite materials and some soft tissues are modeled as incompressible isotropic elastic solids reinforced with preferred directions (see [81], [88] and references therein). Each preferred direction is associated with a family of parallel fibers. Here, two families of fibers are considered. We denote by $\mathbf{M}$ with components $\left(M_{1}, M_{2}, M_{3}\right)$ and $\mathbf{N}$ with components $\left(N_{1}, N_{2}, N_{3}\right)$ the unit vectors in these directions in $\mathcal{B}_{0}$.

The invariants of the right Cauchy-Green deformation tensor, $\mathbf{C}=\mathbf{F}^{\mathrm{T}} \mathbf{F}$, where the symbol ${ }^{\mathrm{T}}$ indicates the transpose of a matrix, most commonly used are the principal invariants (see, for instance [3]), defined by

$$
I_{1}=\mathbf{C}, \quad I_{2}=\frac{1}{2}\left(I_{1}^{2}-\left(\mathbf{C}^{2}\right)\right), \quad I_{3}=\operatorname{det} \mathbf{C} .
$$

The (anisotropic) invariants associated with $\mathbf{M}$ and $\mathbf{C}$ are usually taken 
as

$$
I_{4}=\mathbf{M} \cdot(\mathbf{C M}), \quad I_{5}=\mathbf{M} \cdot\left(\mathbf{C}^{2} \mathbf{M}\right)
$$

For $\mathbf{N}$ and $\mathbf{C}$, the associated invariants are

$$
I_{6}=\mathbf{N} \cdot(\mathbf{C N}), \quad I_{7}=\mathbf{N} \cdot\left(\mathbf{C}^{2} \mathbf{N}\right) .
$$

Finally, the invariant related to the combination of $\mathbf{M}, \mathbf{N}$, and $\mathbf{C}$ is

$$
I_{8}=\mathbf{M} \cdot(\mathbf{C N})(\mathbf{M} \cdot \mathbf{N})
$$

\subsubsection{Material model}

The anisotropic nonlinear elastic strain-energy function $W$ depends on $\mathbf{F}$ through the invariants of the right Cauchy-Green deformation tensor. For incompressible materials, the strain energy function can be written as $W=W\left(I_{1}, I_{2}, I_{4}, I_{5}, I_{6}, I_{7}, I_{8}\right)$ since $I_{3}=1$. If $\mathbf{M}$ and $\mathbf{N}$ are perpendicular then the number of independent invariants is six (see [89] for details). The Cauchy stress is

$$
\boldsymbol{\sigma}=\mathbf{F} \frac{\partial W}{\partial \mathbf{F}}-p \mathbf{I}=\sum_{i=1, i \neq 3}^{8} W_{i} \mathbf{F} \frac{\partial I_{i}}{\partial \mathbf{F}}-p \mathbf{I}
$$

where $p$ is a Lagrange multiplier associated with the incompressibility constraint, the shorthand notations $W_{i}=\partial W / \partial I_{i}, i=1,2,4,5,6,7,8$ have been used and $\mathbf{I}$ is the $3 \times 3$ identity tensor. The Cauchy stress tensor can be written as

$$
\begin{aligned}
& \boldsymbol{\sigma}=2 W_{1} \mathbf{B}+2 W_{2}\left(I_{1} \mathbf{I}-\mathbf{B}\right) \mathbf{B}+2 W_{4} \mathbf{m} \otimes \mathbf{m}+2 W_{5}(\mathbf{m} \otimes \mathbf{B m}+\mathbf{B m} \otimes \mathbf{m}) \\
& +2 W_{6} \mathbf{n} \otimes \mathbf{n}+2 W_{7}(\mathbf{n} \otimes \mathbf{B n}+\mathbf{B n} \otimes \mathbf{n})+W_{8}(\mathbf{m} \otimes \mathbf{n}+\mathbf{n} \otimes \mathbf{m}) \mathbf{M} \cdot \mathbf{N}-p \mathbf{I},(3.6)
\end{aligned}
$$

where $\mathbf{B}=\mathbf{F F}^{\mathrm{T}}, \mathbf{m}=\mathbf{F M}$, and $\mathbf{n}=\mathbf{F N}$. It follows that, in general, the principal directions of stress and strain do not coincide.

In the biomechanics literature, several strain energy functions given by an isotropic elastic material augmented with the so-called reinforcing models can be found. We extend the reinforcing models for one family of fibers 
(see [88] for complete details) to

$$
W=\frac{\mu}{2}\left(I_{1}-3\right)+f_{1}\left(I_{4}\right)+f_{2}\left(I_{6}\right)+g_{1}\left(I_{5}\right)+g_{2}\left(I_{7}\right)+G\left(I_{8}\right)
$$

in order to illustrate the results. This strain energy function captures the essential features of the analysis that follows. We want to establish results related to the kinematical properties of the invariants $I_{4}$ and $I_{6}$ as well as the invariants $I_{5}$ and $I_{7}$. The results allow us to distinguish the effects of the different invariants. The invariant $I_{8}$ is also considered so as to evaluate its influence. For specific details and analysis of the reinforcing models we refer to [81] and [88]. Here, we just mention that the energy function and the stress must vanish in the reference configuration. In Section 3.4, we will further make this clear since a certain strain-energy function is used.

\subsubsection{Linearized incremental equations of motion}

Consider an incompressible, doubly fiber-reinforced, semi-infinite body $\mathcal{B}$ in its unstrained state $\mathcal{B}_{0}$ that occupies the region $X_{2} \geq 0$. Fibers of each family run parallel to each other and perpendicular to the depth direction $X_{2}$, i.e. $M_{2}=0$ and $N_{2}=0$. The body is subjected to a finite pure homogeneous strain with principal directions given by the $X_{i}$-axes. A finitely deformed (pre-stressed) equilibrium state $\mathcal{B}_{e}$ is obtained. A small time-dependent motion is superimposed upon this pre-stressed equilibrium configuration to reach a final material state $\mathcal{B}_{t}$, called current configuration. The vector components of a representative particle are denoted by $X_{i}$, $x_{i}(\mathbf{X}), \tilde{x}_{i}(\mathbf{X}, t)$ in $\mathcal{B}_{0}, \mathcal{B}_{e}$ and $\mathcal{B}_{t}$, respectively. The deformation gradient tensor associated with the deformations $\mathcal{B}_{0} \rightarrow \mathcal{B}_{t}$ and $\mathcal{B}_{0} \rightarrow \mathcal{B}_{e}$ are denoted by $\overline{\mathbf{F}}$ and $\mathbf{F}$, respectively, and are given in component form by

$$
\bar{F}_{i A}=\frac{\partial \widetilde{x}_{i}}{\partial X_{A}}, \quad F_{i A}=\frac{\partial x_{i}}{\partial X_{A}}
$$

It is clear from (3.8) that

$$
\bar{F}_{i A}=\left(\delta_{i j}+u_{i, j}\right) F_{j A},
$$

where $\delta_{i j}$ is the Kronecker operator, $u_{i}(\mathbf{X}, \mathbf{t})$ denotes the small time-dependent displacement associated with the deformation $\mathcal{B}_{e} \rightarrow \mathcal{B}_{t}$ 
and a comma indicates differentiation with respect to the indicated spatial coordinates in $\mathcal{B}_{e}$.

The necessary equations including the linearized equations of motion for anisotropic incompressible materials are summarized. The incremental components of the nominal stress tensor $S_{j i}$ are related to the incremental displacement gradients $u_{k, l}$ by (see $[54 ; 62]$ )

$$
S_{p i}=\mathcal{A}_{p i q j} u_{j, q}+P u_{p, i}-p^{*} \delta_{p i}, \quad i, j, p, q=1,2,3,
$$

where $P$ is the value of $p$ in $\mathcal{B}_{e}, p^{*}=p-P$ is the time-dependent increment of $p$ and the components of the fourth-order elasticity tensor $\mathbf{A}$ for $W=$ $W\left(I_{1}, I_{2}, I_{4}, I_{5}, I_{6}, I_{7}, I_{8}\right)$ are given by (see also Vinh and Merodio 2013b $[75])$

$$
\begin{aligned}
& \mathcal{A}_{p i q j}=F_{p \alpha} F_{q \beta} \frac{\partial^{2} W}{\partial F_{i \alpha} \partial F_{j \beta}} \\
& =F_{p \alpha} F_{q \beta} \sum_{r \in \mathcal{I}} W_{r} \frac{\partial^{2} I_{r}}{\partial F_{i \alpha} \partial F_{j \beta}}+F_{p \alpha} F_{q \beta} \sum_{r, s \in \mathcal{I}} W_{r s} \frac{\partial I_{r}}{\partial F_{i \alpha}} \otimes \frac{\partial I_{s}}{\partial F_{j \beta}}
\end{aligned}
$$

where $W_{r}=\partial W / \partial I_{r}, W_{r s}=\partial^{2} W / \partial I_{r} \partial I_{s}$ and $\mathcal{I}$ is the index set $\{1,2,4,5,6,7,8\}$. The components of the elasticity tensor are

$$
\begin{aligned}
\mathcal{A}_{p i q j} & =2 W_{1} \delta_{i j} B_{p q}+2 W_{2}\left(2 B_{i p} B_{j q}-B_{i q} B_{j p}+I_{1} \delta_{i j} B_{p q}-B_{i j} B_{p q}-\delta_{i j}\left(B^{2}\right)_{p q}\right) \\
+ & 2 W_{4} \delta_{i j} m_{p} m_{q}+2 W_{5}\left[\delta_{i j}\left(m_{p} m_{k} B_{k q}+m_{q} m_{k} B_{k p}\right)\right. \\
+ & \left.B_{i j} m_{p} m_{q}+B_{p q} m_{i} m_{j}+B_{q i} m_{p} m_{j}+B_{p j} m_{q} m_{i}\right]+2 W_{6} \delta_{i j} n_{p} n_{q} \\
+ & 2 W_{7}\left[\delta_{i j}\left(n_{p} n_{k} B_{k q}+n_{q} n_{k} B_{k p}\right)+B_{i j} n_{p} n_{q}+B_{p q} n_{i} n_{j}+2\left(B_{q i} n_{p} n_{j}\right.\right. \\
+ & \left.\left.B_{p j} n_{q} n_{i}\right)\right]+W_{8} \delta_{i j}\left(m_{p} n_{q}+m_{q} n_{p}\right) M_{k} N_{k}+4 W_{11} B_{p i} B_{q j} \\
+ & 4 W_{22}\left(I_{1} B_{i p}-\left(B^{2}\right)_{i p}\right)\left(I_{1} B_{j q}-\left(B^{2}\right)_{j q}\right)+4 W_{44} m_{p} m_{q} m_{i} m_{j} \\
+ & 4 W_{55}\left(B_{i r} m_{p} m_{r}+B_{p r} m_{i} m_{r}\right)\left(B_{j r} m_{q} m_{r}+B_{q r} m_{j} m_{r}\right)+4 W_{66} n_{p} n_{q} n_{i} n_{j} \\
+ & 4 W_{77}\left(B_{i r} n_{p} n_{r}+B_{p r} n_{i} n_{r}\right)\left(B_{j r} n_{q} n_{r}+B_{q r} n_{j} n_{r}\right) \\
+ & W_{88}\left(m_{p} n_{i}+n_{p} m_{i}\right)\left(m_{q} n_{j}+n_{q} m_{j}\right) M_{k} N_{k} M_{t} N_{t} \\
+ & 4 W_{12}\left[B_{i p}\left(I_{1} B_{j q}-\left(B^{2}\right)_{j q}\right)+B_{j q}\left(I_{1} B_{i p}-\left(B^{2}\right)_{i p}\right)\right]
\end{aligned}
$$




$$
\begin{aligned}
& +4 W_{14}\left(B_{p i} m_{q} m_{j}+B_{q j} m_{p} m_{i}\right)+4 W_{15}\left[B_{p i}\left(B_{j r} m_{q} m_{r}+B_{q r} m_{j} m_{r}\right)\right. \\
& \left.+B_{q j}\left(B_{i r} m_{p} m_{r}+B_{p r} m_{i} m_{r}\right)\right]+4 W_{16}\left(B_{p i} n_{q} n_{j}+B_{q j} n_{p} n_{i}\right) \\
& +4 W_{17}\left[B_{p i}\left(B_{j r} n_{q} n_{r}+B_{q r} n_{j} n_{r}\right)+B_{q j}\left(B_{i r} n_{p} n_{r}+B_{p r} n_{i} n_{r}\right)\right] \\
& +2 W_{18}\left[B_{p i}\left(m_{q} n_{j}+n_{q} m_{j}\right) M_{k} N_{k}+B_{q j}\left(m_{p} n_{i}+n_{p} m_{i}\right) M_{k} N_{k}\right] \\
& +4 W_{24}\left[\left(I_{1} B_{i p}-\left(B^{2}\right)_{i p}\right) m_{j} m_{q}+\left(I_{1} B_{j q}-\left(B^{2}\right)_{j q}\right) m_{i} m_{p}\right] \\
& +4 W_{25}\left[( I _ { 1 } B _ { i p } - ( B ^ { 2 } ) _ { i p } ) \left[m_{q} B_{j r} m_{r}\right.\right. \\
& \left.\left.+m_{j} B_{q r} m_{r}\right]+\left(I_{1} B_{j q}-\left(B^{2}\right)_{j q}\right)\left[m_{i} B_{p r} m_{r}+m_{p} B_{i r} m_{r}\right]\right] \\
& +4 W_{26}\left[\left(I_{1} B_{i p}-\left(B^{2}\right)_{i p}\right) n_{j} n_{q}+\left(I_{1} B_{j q}-\left(B^{2}\right)_{j q}\right) n_{i} n_{p}\right] \\
& +4 W_{27}\left[\left(I_{1} B_{i p}-\left(B^{2}\right)_{i p}\right)\left(n_{q} B_{j r} n_{r}+n_{j} B_{q r} n_{r}\right)\right. \\
& \left.+\left(I_{1} B_{j q}-\left(B^{2}\right)_{j q}\right)\left(n_{i} B_{p r} n_{r}+n_{p} B_{i r} n_{r}\right)\right] \\
& +2 W_{28}\left[B_{i p} I_{1}\left(m_{q} n_{j}+n_{q} m_{j}\right)-B_{p \gamma} B_{\gamma i}\left(m_{q} n_{j}+n_{q} m_{j}\right)\right. \\
& \left.+B_{q j} I_{1}\left(m_{p} m_{i}+n_{p} m_{i}\right)-B_{q \gamma} B_{\gamma j}\left(m_{p} n_{i}+n_{p} m_{i}\right)\right] M_{k} N_{k} \\
& +4 W_{45}\left[m_{p} m_{i}\left(B_{j r} m_{q} m_{r}+B_{q r} m_{j} m_{r}\right)+m_{q} m_{j}\left(B_{i r} m_{p} m_{r}+B_{p r} m_{i} m_{r}\right)\right] \\
& +4 W_{46}\left(m_{p} m_{i} n_{q} n_{j}+n_{p} n_{i} m_{q} m_{j}\right)+4 W_{47}\left[m_{p} m_{i}\left(B_{j r} n_{q} n_{r}+B_{q r} n_{j} n_{r}\right)\right. \\
& \left.+m_{q} m_{j}\left(B_{i r} n_{p} n_{r}+B_{p r} n_{i} n_{r}\right)\right]+4 W_{48}\left(m_{p} m_{i}\left(m_{q} n_{j}+n_{q} m_{j}\right) M_{k} N_{k}\right. \\
& \left.+m_{q} m_{j}\left(m_{p} n_{i}+n_{p} m_{i}\right) M_{k} N_{k}\right)+4 W_{56}\left[n_{q} n_{i}\left(B_{i r} m_{p} m_{r}+B_{p r} m_{i} m_{r}\right)\right. \\
& \left.+n_{p} n_{j}\left(B_{i r} m_{q} m_{r}+B_{q r} m_{j} m_{r}\right)\right]+4 W_{57}\left(B_{i r} m_{p} m_{r}+B_{p r} m_{i} m_{r}\right)\left(B_{j r} n_{q} n_{r}\right. \\
& \left.+B_{q r} n_{j} n_{r}\right)+2 W_{58}\left[\left(B_{i r} m_{p} m_{r}+B_{p r} m_{i} m_{r}\right)\left(m_{q} n_{j}+n_{q} m_{j}\right)+\left(B_{j r} m_{q} m_{r}\right.\right. \\
& \left.\left.+B_{q r} m_{j} m_{r}\right)\left(m_{p} n_{i}+n_{p} m_{i}\right)\right] M_{k} N_{k}+4 W_{67}\left[n_{p} n_{i}\left(B_{j r} n_{q} n_{r}+B_{q r} n_{j} n_{r}\right)\right. \\
& \left.+n_{q} n_{j}\left(B_{i r} n_{p} n_{r}+B_{p r} n_{i} n_{r}\right)\right]+2 W_{68}\left[n_{p} n_{i}\left(m_{q} n_{j}+n_{q} m_{j}\right)+n_{q} n_{j}\left(m_{p} n_{i}+n_{p} m_{i}\right)\right] \\
& +2 W_{78}\left[\left(B_{i r} n_{p} n_{r}+B_{p r} n_{i} n_{r}\right)\left(m_{q} n_{j}+n_{q} m_{j}\right)\right. \\
& \left.+\left(B_{j r} n_{q} n_{r}+B_{q r} n_{j} n_{r}\right)\left(m_{p} n_{i}+n_{p} m_{i}\right)\right]
\end{aligned}
$$

with $B_{i j}=F_{i k} F_{j k}$ and $I_{1}=B_{k k}$.

It is clear that $\mathcal{A}_{p i q j}=\mathcal{A}_{q j p i}$. In general, the elasticity tensor $\mathbf{A}$ has at most 45 non-zero components.

The fibers $\mathbf{M}$ and $\mathbf{N}$ in $\mathcal{B}_{0}$ make angles $\gamma$ and $\delta$, respectively, with $O X_{1}$ and the angles are measured in opposite senses relative to that axes. Since the deformation gradient $\mathbf{F}$ is $\mathbf{F}=\operatorname{diag}\left(\lambda_{1}, \lambda_{2}, \lambda_{3}\right)$, where $\lambda_{k}$ are the principal 


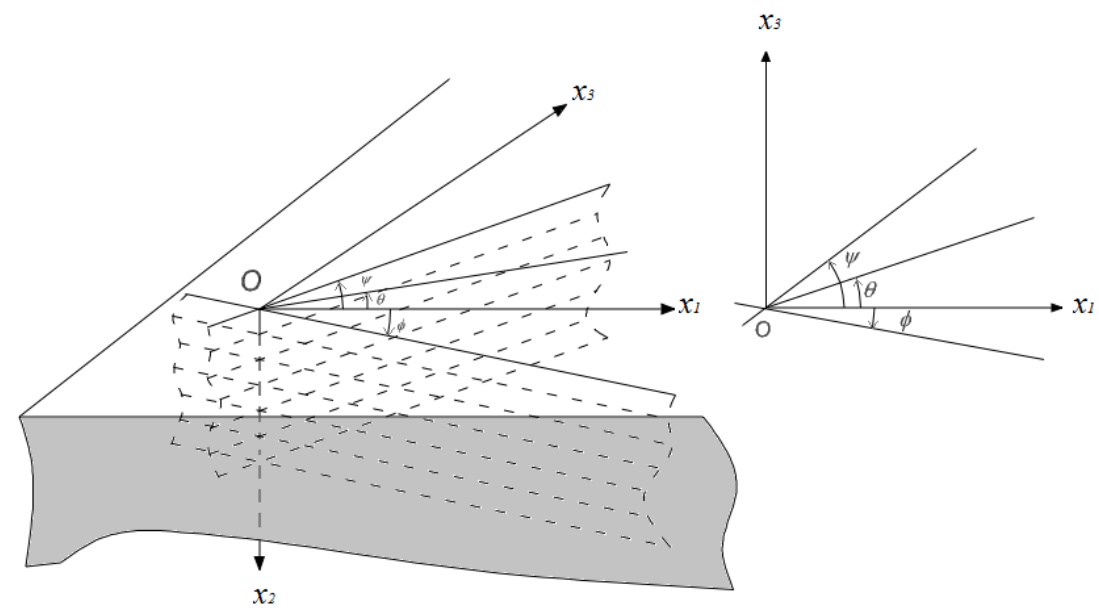

Figure 3.1: The figure on the left shows at a point $O$ in the free surface of the pre-stressed half space: i) the principal axes of the primary pure homogeneous strain $\left(x_{i}\right.$-axes $)$ ii) the two directions in that configuration characterizing the two families of fibers (given by $\psi$ and $\phi$ ) as well as the fibers of each family (dashed lines) along the depth direction $\left(x_{2}\right.$-axis) and iii) the propagation direction of the wave (given by $\theta$ ). Fibers of each family are located throughout the whole half space and run parallel to each other and perpendicular to the depth direction. The figure on the right is a view from the top. It further clarifies that the angles $\psi$ and $\phi$ are meassured in opposite senses relative to the $x_{1}$-axis.

stretches of the deformation, it follows that that the components of $\mathbf{m}$ and $\mathbf{n}$ are

$$
\begin{array}{r}
m_{1}=\lambda_{1} \cos \gamma, \quad n_{1}=\lambda_{1} \cos \delta, \quad m_{2}=M_{2}=m_{2}=N_{2}=0, \\
m_{3}=\lambda_{3} \sin \gamma, \quad n_{3}=-\lambda_{3} \sin \delta .
\end{array}
$$

The vectors $\mathbf{m}$ and $\mathbf{n}$ in $\mathcal{B}_{e}$ make angles $\psi$ and $\phi$, respectively, with $O x_{1}$, which, using (3.13), are given by

$$
\tan \psi=\lambda_{3} / \lambda_{1} \tan \gamma, \tan \phi=\lambda_{3} / \lambda_{1} \tan \delta
$$

Given $\mathbf{F}$, one can assume that either the set of angles $\psi$ and $\phi$ or the set of angles $\gamma$ and $\delta$ is known (see Figure 3.1).

We further particularize the elasticity tensor to the strain-energy function 
(3.7), i.e. using (3.7) and (3.11) we write

$$
\begin{aligned}
& \mathcal{A}_{p i q j}=\mu \delta_{i j} B_{p q} \\
+ & \left.2 f_{1}^{\prime}\left(I_{4}\right) m_{p} m_{q}+2 f_{2}^{\prime}\left(I_{6}\right) n_{p} n_{q}\right]+4 f_{1}^{\prime \prime}\left(I_{4}\right) m_{i} m_{j} m_{p} m_{q}+4 f_{2}^{\prime \prime}\left(I_{6}\right) n_{i} n_{j} n_{p} n_{q} \\
+ & 2 g_{1}^{\prime}\left(I_{5}\right)\left[\delta_{i j}\left(m_{p} B_{q r} m_{r}+m_{q} B_{p r} m_{r}\right)+B_{i j} m_{p} m_{q}+m_{i} m_{j} B_{p q}+B_{i q} m_{j} m_{p}+B_{p j} m_{i} m_{q}\right] \\
+ & 2 g_{2}^{\prime}\left(I_{7}\right)\left[\delta_{i j}\left(n_{p} B_{q r} n_{r}+n_{q} B_{p r} n_{r}\right)+B_{i j} n_{p} n_{q}+n_{i} n_{j} B_{p q}+B_{i q} n_{j} n_{p}+B_{p j} n_{i} n_{q}\right] \\
+ & 4 g_{1}^{\prime \prime}\left(I_{5}\right)\left(m_{p} B_{i r} m_{r}+m_{i} B_{p r} m_{r}\right)\left(m_{q} B_{j r} m_{r}+m_{j} B_{q r} m_{r}\right) \\
+ & 4 g_{2}^{\prime \prime}\left(I_{7}\right)\left(n_{p} B_{i r} n_{r}+n_{i} B_{p r} n_{r}\right)\left(n_{q} B_{j r} n_{r}+n_{j} B_{q r} n_{r}\right) \\
+ & G^{\prime} \delta_{i j}\left(m_{p} n_{q}+m_{q} n_{p}\right) M_{k} N_{k}+G^{\prime \prime}\left(m_{p} n_{i}+n_{p} m_{i}\right)\left(m_{q} n_{j}+n_{q} m_{j}\right) M_{k} N_{k} M_{t} N_{t} .
\end{aligned}
$$

In the absence of body forces, the incremental equations of motion are (see [82])

$$
S_{i j, i}=\rho \ddot{u}_{j}
$$

where a dot indicates differentiation with respect to time $t$. The incremental version of incompressibility is (see [82])

$$
u_{i, i}=0
$$

In what follows, we rewrite these equations for the case of a Rayleigh wave using the Stroh formulation. In addition, using (3.11) we point out that there are only 25 non-zero components of the elasticity tensor $\mathcal{A}$, namely $\mathcal{A}_{i i i i}, \mathcal{A}_{i i j j}, \mathcal{A}_{i j i j}, \mathcal{A}_{i j j i}, \mathcal{A}_{i i 13}, \mathcal{A}_{i i 31}, \mathcal{A}_{2312}, \mathcal{A}_{2321}, \mathcal{A}_{3212}, \mathcal{A}_{3221}$ $(i, j=1,2,3, i \neq j)$.

\subsection{Surface waves}

The analysis is particularized for Rayleigh waves propagating in a principal plane of the pre-strain, the plane $x_{2}=0$, but not in general in a principal direction. The incremental equation of motion can be cast as a homogeneous linear system of six first-order differential equations. 


\subsubsection{The Stroh formulation}

We consider a Rayleigh wave traveling with speed $c$ and with its wave vector $\mathbf{k}$ lying in the $\left(x_{1}, x_{3}\right)$ plane. The wave makes an angle $\theta$ with the $x_{1}$-direction and decays in the $x_{2}$-direction. Then, the displacements and stresses of the Rayleigh wave are written (see [84]) as

$$
u_{n}=U_{n}(y) e^{i k\left(x_{1} c_{\theta}+x_{3} s_{\theta}-c t\right)}, S_{2 n}=i k z_{n}(y) e^{i k\left(x_{1} c_{\theta}+x_{3} s_{\theta}-c t\right)}, n=1,2,3,
$$

respectively, where $y=k x_{2}, c_{\theta}=\cos \theta, s_{\theta}=\sin \theta$, and $k=|\mathbf{k}|$ is the wave number.

Using (3.18), together with (3.10), (3.16) and (3.17), one can write

$$
\boldsymbol{\xi}^{\prime}=i \mathbf{N} \boldsymbol{\xi}, \quad 0 \leq y<+\infty
$$

where the prime now signifies differentiation with respect to $y$ and

$$
\boldsymbol{\xi}=\left[\begin{array}{l}
\mathbf{u} \\
\mathbf{z}
\end{array}\right], \mathbf{u}=\left[\begin{array}{c}
U_{1} \\
U_{2} \\
U_{3}
\end{array}\right], \mathbf{z}=\left[\begin{array}{c}
z_{1} \\
z_{2} \\
z_{3}
\end{array}\right], \mathbf{N}=\left[\begin{array}{cc}
\mathbf{N}_{1} & \mathbf{N}_{2} \\
\mathbf{K} & \mathbf{N}_{1}^{T}
\end{array}\right]
$$

in which the matrices $\mathbf{N}_{1}, \mathbf{N}_{2}, \mathbf{K}$ are defined by

$$
\mathbf{N}_{1}=\left[\begin{array}{ccc}
0 & f_{1} & 0 \\
-c_{\theta} & 0 & -s_{\theta} \\
0 & f_{2} & 0
\end{array}\right], \mathbf{N}_{2}=\left[\begin{array}{ccc}
d_{1} & 0 & -d_{13} \\
0 & 0 & 0 \\
-d_{13} & 0 & d_{3}
\end{array}\right], \quad \mathbf{K}=\left[\begin{array}{ccc}
h_{1} & 0 & h_{2} \\
0 & h_{3} & 0 \\
h_{2} & 0 & h_{4}
\end{array}\right]
$$

where

$$
\begin{aligned}
& d=\mathcal{A}_{2121} \mathcal{A}_{2323}-\mathcal{A}_{2123}^{2}, \quad d_{1}=\frac{\mathcal{A}_{2323}}{d}, \quad d_{13}=\frac{\mathcal{A}_{2123}}{d}, \quad d_{3}=\frac{\mathcal{A}_{2121}}{d}, \\
& f_{1}=a_{11} c_{\theta}+a_{13} s_{\theta}, \quad f_{2}=a_{31} c_{\theta}+a_{33} s_{\theta}, \\
& h_{1}=\rho c^{2}-b_{111} c_{\theta}^{2}-b_{113} c_{\theta} s_{\theta}-b_{133} s_{\theta}^{2}, \quad h_{2}=-b_{311} c_{\theta}^{2}-b_{313} c_{\theta} s_{\theta}-b_{333} s_{\theta}^{2}, \\
& h_{3}=\rho c^{2}-e_{11} c_{\theta}^{2}-e_{13} c_{\theta} s_{\theta}-e_{33} s_{\theta}^{2}, \quad h_{4}=\rho c^{2}-d_{311} c_{\theta}^{2}-d_{313} c_{\theta} s_{\theta}-d_{333} s_{\theta}^{2},
\end{aligned}
$$


and

$$
\begin{aligned}
a_{11} & =\left(\mathcal{A}_{2123} \mathcal{A}_{1223}-\mathcal{A}_{2323} \mathcal{A}_{1221}^{*}\right) / d, a_{13}=\left(\mathcal{A}_{2123} \mathcal{A}_{2332}^{*}-\mathcal{A}_{2323} \mathcal{A}_{2132}\right) / d \\
a_{31} & =\left(\mathcal{A}_{2123} \mathcal{A}_{1221}^{*}-\mathcal{A}_{2121} \mathcal{A}_{2312}\right) / d, a_{33}=\left(\mathcal{A}_{2123} \mathcal{A}_{2132}-\mathcal{A}_{2121} \mathcal{A}_{2332}^{*}\right) / d \\
b_{111} & =\left(\mathcal{A}_{1111}^{*}+\mathcal{A}_{2222}^{*}-2 \mathcal{A}_{1122}\right), b_{113}=2\left(\mathcal{A}_{1131}-\mathcal{A}_{2231}\right), b_{133}=\mathcal{A}_{3131}, \\
b_{133} & =\left(\mathcal{A}_{2222}^{*}+\mathcal{A}_{1331}^{*}+\mathcal{A}_{1133}-\mathcal{A}_{1122}-\mathcal{A}_{3322}\right), \\
b_{311} & =\left(\mathcal{A}_{1113}-\mathcal{A}_{2213}\right), b_{333}=\left(\mathcal{A}_{3133}-\mathcal{A}_{3122}\right), \\
d_{311}=\mathcal{A}_{1313}, d_{313}=2\left(\mathcal{A}_{1333}-\mathcal{A}_{1322}\right), d_{333}=\left(\mathcal{A}_{3333}^{*}+\mathcal{A}_{2222}^{*}-2 \mathcal{A}_{2233}\right), & \left(\mathcal{A}_{1212}+a_{11} \mathcal{A}_{1221}^{*}+a_{31} \mathcal{A}_{1223}\right), e_{33}=\left(\mathcal{A}_{3232}+a_{13} \mathcal{A}_{3221}+a_{33} \mathcal{A}_{2332}^{*}\right), \\
e_{11}=\left(2 \mathcal{A}_{1232}+a_{11} \mathcal{A}_{3221}+a_{13} \mathcal{A}_{1221}^{*}+a_{31} \mathcal{A}_{3223}^{*}+a_{33} \mathcal{A}_{1223}\right) &
\end{aligned}
$$

Here the notation $A_{p i q j}^{*}=A_{p i q j}+P \delta_{i j} \delta_{p q}$ has been introduced. Equation (3.19) is the so-called Stroh formulation (see [34]). The decay condition is expressed as

$$
\boldsymbol{\xi}(+\infty)=0
$$

The boundary condition of zero incremental traction using the expression given for $S_{2 n}$ in (3.18) means that

$$
\mathbf{z}(0)=0
$$

In passing, we note that our formulation particularized for transversely isotropic materials and isotropic materials coincides with the ones given in [62] and [84], respectively. In particular, for instance, the matrices $\mathbf{N}_{1}, \mathbf{N}_{2}$ and $\mathbf{K}$ in (3.21) particularized for isotropic materials coincide, respectively, with the matrices $\mathbf{N}_{1}, \mathbf{N}_{2}$ and $\mathbf{N}_{3}+X \mathbf{I}$, where $X=\rho c^{2}$, given by (2.9) and (2.10) in [84].

\subsubsection{Implicit secular equation}

The explicit secular equation is given by (see [84] and [65] for complete details)

$$
v \omega_{I}-\left(u-\omega_{I I}\right) \omega_{I I I}=0,
$$

in which

$$
\omega_{I}=-\left(s_{1}+s_{2}+s_{3}\right), \omega_{I I}=s_{1} s_{2}+s_{2} s_{3}+s_{3} s_{1}, \omega_{I I I}=-s_{1} s_{2} s_{3},
$$


where $s_{1} s_{2}, s_{3}$ are the eigenvalues of the Stroh matrix $\mathbf{N}$ with positive imaginary parts and $u, v$ are defined as

$$
\begin{aligned}
& u=\left(\frac{f_{1}^{2}}{h_{3}}-d_{1}\right) h_{1}+\left(\frac{f_{2}^{2}}{h_{3}}-d_{3}\right) h_{4}+2\left(\frac{f_{1} f_{2}}{h_{3}}+d_{13}\right) h_{2} \\
& v=\left(h_{1} h_{4}-h_{2}^{2}\right)\left[d_{1} d_{3}-\frac{d_{3} f_{1}^{2}+d_{1} f_{2}^{2}}{h_{3}}-\frac{2 d_{13} f_{1} f_{2}}{h_{3}}-d_{13}^{2}\right]
\end{aligned}
$$

Equation (3.25) is called the implicit secular equation (see also [84] and [62]) because the expressions for the $\omega_{I}, \omega_{I I}, \omega_{I I I}$ in terms of $X$ are unkown. A Rayleigh wave exists with speed $c=\sqrt{X / \rho}$ if and only if (3.25) is satisfied.

\subsubsection{Explicit secular equation}

In this section we derive the explicit secular equation of the wave using the method of polarization vector (see [90], [44] and [91] for instance). Using (3.19), (3.23), (3.24) and that $\mathbf{N}_{2}$ and $\mathbf{K}$ are symmetric, one can write

$$
\overline{\mathbf{u}}^{\mathrm{T}}(0) \mathbf{K}^{(n)} \mathbf{u}(0)=0 \forall n \in \mathbf{Z},
$$

where $\mathbf{K}^{(n)}$ is defined as

$$
\mathbf{N}^{n}=\left[\begin{array}{ll}
\mathbf{N}_{1}^{(n)} & \mathbf{N}_{2}^{(n)} \\
\mathbf{K}^{(n)} & \mathbf{N}_{4}^{(n)}
\end{array}\right]
$$

From (3.28) the explicit secular equation is obtained as

$$
\left|\mathbf{K}_{2}, \mathbf{K}_{3}, \mathbf{K}_{1}\right|^{2}+4\left|\mathbf{K}_{2}, \mathbf{K}_{3}, \mathbf{K}_{4}\right|\left|\mathbf{K}_{2}, \mathbf{K}_{1}, \mathbf{K}_{4}\right|=0
$$

where

$$
\mathbf{K}_{1}=\left[\begin{array}{c}
K_{11}^{(-1)} \\
K_{11}^{(1)} \\
K_{11}^{(3)}
\end{array}\right], \quad \mathbf{K}_{2}=\left[\begin{array}{c}
K_{22}^{(-1)} \\
K_{22}^{(1)} \\
K_{22}^{(3)}
\end{array}\right], \quad \mathbf{K}_{3}=\left[\begin{array}{c}
K_{33}^{(-1)} \\
K_{33}^{(1)} \\
K_{33}^{(3)}
\end{array}\right], \quad \mathbf{K}_{4}=\left[\begin{array}{c}
K_{13}^{(-1)} \\
K_{13}^{(1)} \\
K_{13}^{(3)}
\end{array}\right]
$$


in which $K_{i j}^{(n)}$ are entries of the matrix $\mathbf{K}^{(n)}$

$$
\begin{aligned}
K_{11}^{(1)} & =h_{1}, \quad K_{13}^{(1)}=h_{2}, \quad K_{22}^{(1)}=h_{3}, \quad K_{33}^{(1)}=h_{4}, \\
K_{11}^{(3)}= & d_{1} h_{1}^{2}+d_{3} h_{2}^{2}-2 d_{13} h_{1} h_{2}-2\left(f_{1} h_{1}+f_{2} h_{2}\right) c_{\theta}+h_{3} c_{\theta}^{2}, \\
K_{13}^{(3)}= & d_{1} h_{1} h_{2}-d_{13}\left(h_{2}^{2}+h_{1} h_{4}\right)+d_{3} h_{2} h_{4} \\
& -\left(f_{1} h_{1}+f_{2} h_{2}\right) s_{\theta}-\left(f_{1} h_{2}+f_{2} h_{4}\right) c_{\theta}+h_{3} s_{\theta} c_{\theta}, \\
K_{22}^{(3)}= & f_{1}^{2} h_{1}+2 f_{1} f_{2} h_{2}-2 f_{1} h_{3} c_{\theta}+f_{2}^{2} h_{4}-2 f_{2} h_{3} s_{\theta}, \\
K_{33}^{(3)}= & d_{1} h_{2}^{2}+d_{3} h_{4}^{2}-2 d_{13} h_{2} h_{4}-2\left(f_{1} h_{2}+f_{2} h_{4}\right) s_{\theta}+h_{3} s_{\theta}^{2}, \\
K_{11}^{(-1)}= & \left(d_{3} h_{3}-f_{2}^{2}\right)\left(h_{4} c_{\theta}^{2}-2 h_{2} c_{\theta} s_{\theta}+h_{1} s_{\theta}^{2}\right), \\
K_{13}^{(-1)}= & \left(d_{13} h_{3}+f_{1} f_{2}\right)\left(h_{4} c_{\theta}^{2}-2 h_{2} c_{\theta} s_{\theta}+h_{1} s_{\theta}^{2}\right), \\
K_{22}^{(-1)}= & {\left[2 d_{13} f_{1} f_{2}+d_{1} f_{2}^{2}+d_{13}^{2} h_{3}+d_{3}\left(f_{1}^{2}-d_{1} h_{3}\right)\right]\left(h_{1} h_{4}-h_{2}^{2}\right), } \\
K_{33}^{(-1)} & =\left(d_{1} h_{3}-f_{1}^{2}\right)\left(h_{4} c_{\theta}^{2}-2 h_{2} c_{\theta} s_{\theta}+h_{1} s_{\theta}^{2}\right) .
\end{aligned}
$$

Here the notation $A_{p i q j}^{*}=A_{p i q j}+P \delta_{i j} \delta_{p q}$ has been introduced. Equation (3.30) is the explicit secular equation. This is a cumbersome polynomial of degree 12 in $X=\rho c^{2}$ (see [62]).

In order to illustrate the results further we consider some particular strainenergy functions.

\subsection{Numerical results}

A modified version of the well known Gasser-Ogden-Holzapfel (GOH) model (see [77]) is adopted. In particular, we consider that

$$
W=\frac{\mu}{2}\left(I_{1}-3\right)+\frac{k_{1}}{2 k_{2}} \sum_{i=4,5,6,7}\left\{\exp \left[k_{2}\left(I_{i}-1\right)^{2}\right]-1\right\}+\frac{k_{3}}{2}\left(I_{8}-I_{8}^{(0)}\right)^{2}
$$

where $\mu, k_{1}, k_{2}$ and $k_{3}$ are positive constants and $I_{8}^{(0)}=(\mathbf{M} \cdot \mathbf{N})^{2}$ is the value of $I_{8}$ in the reference configuration. The GOH model is given by (3.33) with no dependence on $I_{5}, I_{7}$ and $I_{8}$. Furthermore, it is assumed that the fibers contribute to the strain-energy function when these are elongated. Here, we use (3.33) as a prototype to show the robustness of the methodology herein regardless of this last statement. Nevertheless, and in passing, we mention that lately there has been some discussion regarding the tension-compression switch in these models and we refer to [92] for 
further details. It is easy to check that the strain energy is zero in the undeformed configuration as well as the stress tensor. We specialize (3.33) to some special models and compare the results with the ones obtained for the neo-Hookean material, whose energy function is

$$
W_{0}=\frac{\mu}{2}\left(I_{1}-3\right)
$$

Hence, we treat in turn the following cases:

i) the strain energy function (3.33) just with the invariants $I_{1}, I_{4}, I_{6}$

$$
W^{(1)}\left(I_{1}, I_{4}, I_{6}\right)=\frac{\mu}{2}\left(I_{1}-3\right)+\frac{k_{1}}{2 k_{2}} \sum_{i=4,6}\left[e^{k_{2}\left(I_{i}-1\right)^{2}}-1\right]
$$

ii) similarly, the strain energy function (3.33) just with the invariants $I_{1}, I_{5}, I_{7}$

$$
W^{(2)}\left(I_{1}, I_{5}, I_{7}\right)=\frac{\mu}{2}\left(I_{1}-3\right)+\frac{k_{1}}{2 k_{2}} \sum_{i=5,7}\left[e^{k_{2}\left(I_{i}-1\right)^{2}}-1\right] .
$$

In Figure 3.2, values of $x=\rho c^{2} / \mu$ vs $\theta \in[0, \pi / 2]$ obtained using (3.30) are plotted for different strain-energy functions under two conditions, namely $\lambda_{1}=1.3, \lambda_{2}=1$ and $\lambda_{3}=1 / \lambda_{1}$ (right-hand plot figure) and $\lambda_{1}=1.2, \lambda_{2}=$ 1 and $\lambda_{3}=1 / \lambda_{1}$ (left-hand plot figure). In both cases, the dotted-dashed curve is associated with (3.33) for $\gamma=\pi / 6, \delta=\pi / 3, k_{1}=k_{3}=0.5 \mu$ and $k_{2}=0.5$. The values of these parameters are used accordingly in the models (3.34), (3.35) and (3.36). The curves associated to the neo-Hookean model have their maximum value at $\theta=0$, which is expected for an isotropic model. That is not the case for the non-isotropic models since the principal directions of stress and strain do not coincide. Other parameters could be used as well as other angles for the fibers. Furthermore, the influence on the wave speed of the isotropic base model introduced by the invariants $I_{5}$ and $I_{7}$ (the model (3.36)) is stronger than the one given by the invariants $I_{4}$ and $I_{6}$ (the model (3.35)). This result was shown in chapter 2 for the analysis of transversely isotropic materials with one family of fibers.

In Figure 3.3, the same analysis is developed for $\gamma=\delta=\pi / 4$ (perpendicular). Under these circumstances (the two families of fibers are symmetric with respect to the $O X_{1}$ axis), it follows that $I_{4}=I_{6}$ and 

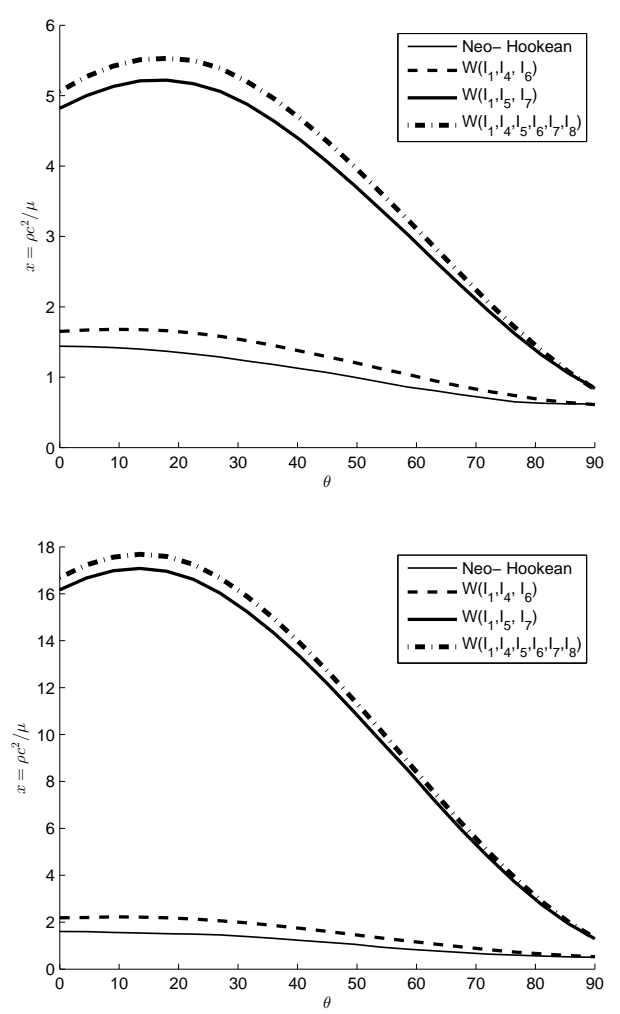

Figure 3.2: In the two plots, the curves show the dependence of $x=\rho c^{2} / \mu$ on $\theta \in[0, \pi / 2]$ obtained using (3.30) for (3.33), the dotted-dashed curve, (3.34), the thin solid curve, as well as (3.35) and (3.36), the dashed and thick solid curves, respectively. For the different calculations, we have taken, accordingly for each model, $\gamma=\pi / 6, \delta=\pi / 3, k_{1}=k_{3}=0.5 \mu, k_{2}=0.5$. The principal stretches are $\lambda_{1}=\lambda=1.2, \lambda_{2}=1, \lambda_{3}=1 / \lambda_{1}$ (left-hand plot); $\lambda_{1}=1.3, \lambda_{2}=1$ and $\lambda_{3}=1 / \lambda_{1}$ (right-hand plot). 

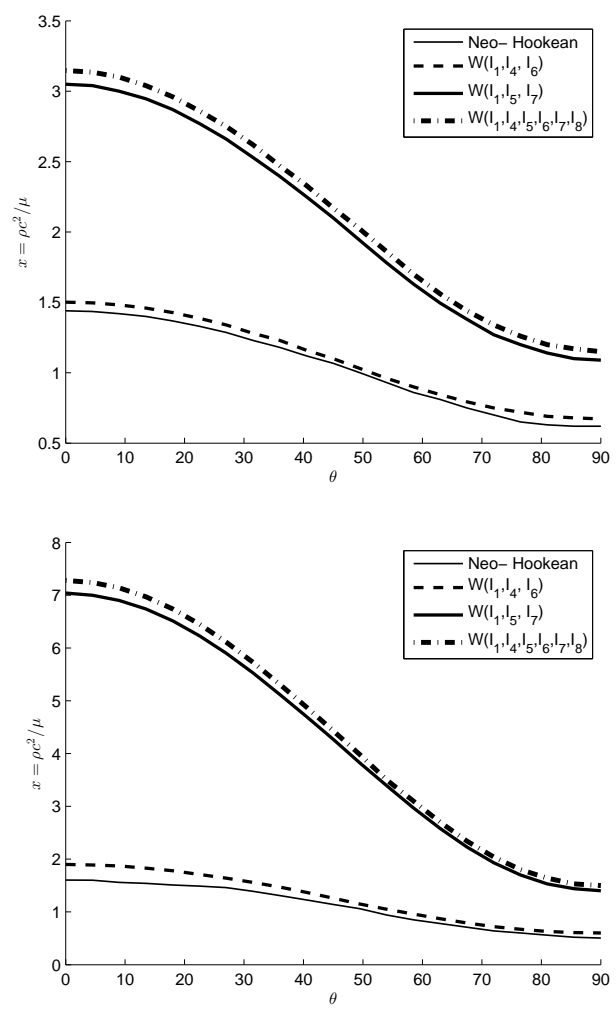

Figure 3.3: The curves show in the two plots the dependence of $x=\rho c^{2} / \mu$ on $\theta \in$ $[0, \pi / 2]$ as given by (3.30) for (3.33), the dotted-dashed curve, as well as (3.35) and (3.36), the dashed and thick solid curves, respectively. The parameters of the different models have been taken as $\gamma=\pi / 4, \delta=\pi / 4, k_{1}=k_{3}=0.5 \mu$ and $k_{2}=0.5$. The principal stretches are $\lambda_{1}=1.2, \lambda_{2}=1, \lambda_{3}=1 / \lambda_{1}$ (left-hand plot) and b) $\lambda_{1}=1.3, \lambda_{2}=1, \lambda_{3}=1 / \lambda_{1}$ (right-hand plot). Results for the neo-Hookean model (3.34), the thin solid curve, are also shown for comparison.

$I_{5}=I_{7}$ and, furthermore, the principal directions of strain and stress coincide. Hence, each curve in Figure 3.3 has its maximum value at $\theta=0$.

Corresponding plots to the ones given in Figure 3.2 are shown in Figures 3.4 and 3.5 for different angles (not perpendicular) of $\gamma$ and $\delta$. In particular $\gamma=\pi / 6$ and $\delta=\pi / 4$ in Figure 3.4 and $\gamma=\pi / 6$ and $\delta=\pi / 6$ in Figure 3.5. The influence of the term in (3.33) that includes the invariant $I_{8}$ on the surface wave speed of the isotropic model is not as significant as the influence of the other non-isotropic invariants. Indeed, results may be different for other strain-energy functions and other deformations.

We consider now that the elastic half-space is initially under uniaxial 

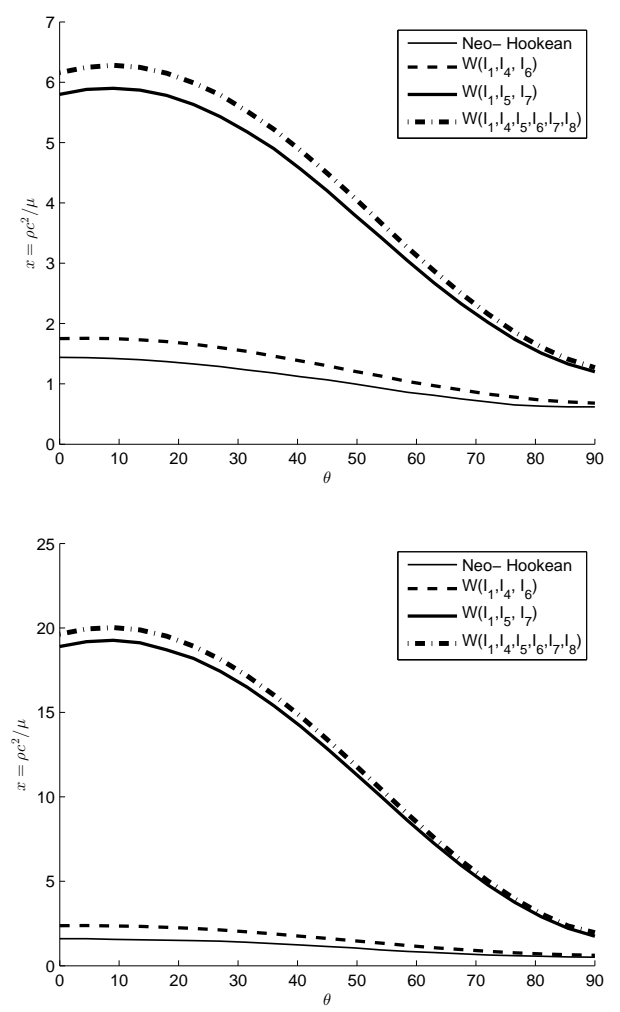

Figure 3.4: Corresponding plots to the ones given in Figure 3.2 for $\gamma=\pi / 6$ and $\delta=\pi / 4$. 

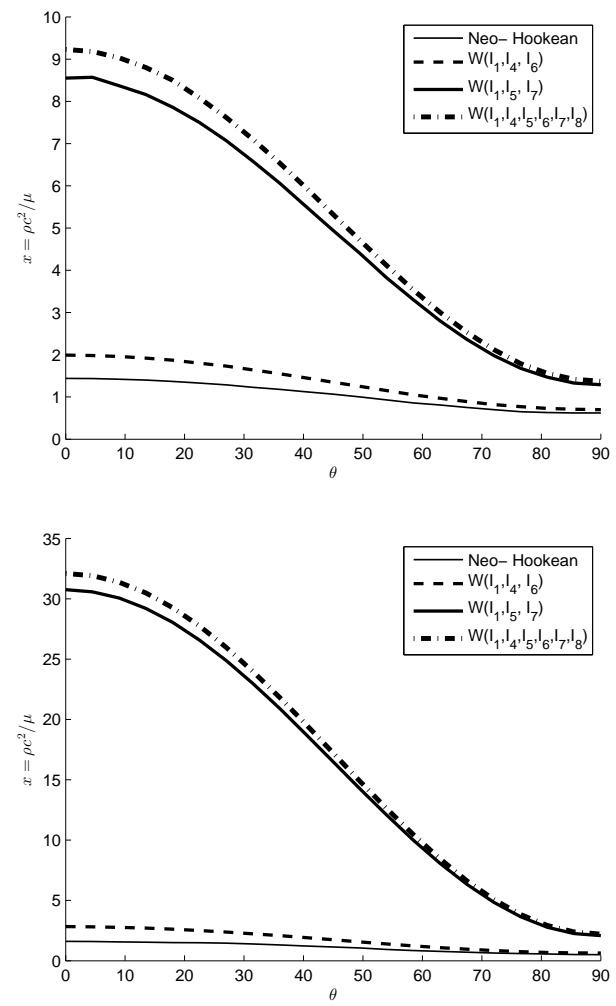

Figure 3.5: Corresponding plots to the ones given in Figure 3.2 for $\gamma=\pi / 6$ and $\delta=\pi / 6$. 


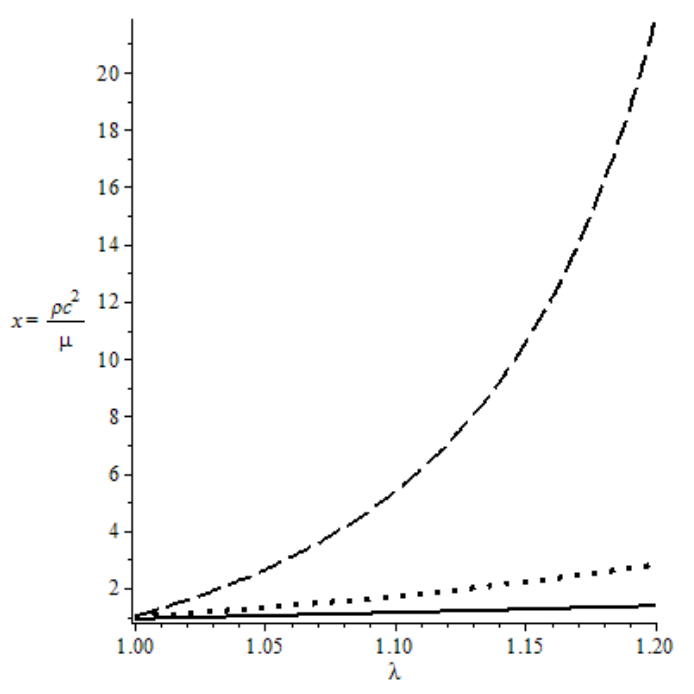

Figure 3.6: Under uniaxial tension along the $X_{1}$-axis with $\gamma=\delta=\pi / 4$ and waves propagating along the $x_{1}$-axis, the Figure shows the dependence of $x=$ $\rho c^{2} / \mu$ on $\lambda$ as given by (3.30) for (3.35), the dotted curve, and (3.36), the dashed curve. Results for the neo-Hookean model (3.34), the solid curve, are also shown for comparison. The parameters for the different models are $k_{1}=0.5 \mu$ and $k_{2}=0.5$.

tension along the $X_{1}$-axis

$$
x_{1}=\lambda X_{1}, x_{2}=\lambda^{-1 / 2} X_{2}, x_{3}=\lambda^{-1 / 2} X_{3}, \lambda>0, \lambda=\text { const. }
$$

In addition, the surface waves propagate in the $x_{1}$-direction and the two families of fibers are symmetrically disposed with respect to the $X_{1}$-axis, in particular, $\gamma=\delta=\pi / 4$. In Figure 3.6, values of $x=\rho c^{2} / \mu$ vs $\lambda$ obtained using (3.30) are shown for the neo-Hookean model (3.34), the solid curve, as well as for (3.35) and (3.36), the dotted and dashed curves, respectively. The parameters for the different models are $k_{1}=0.5 \mu$ and $k_{2}=0.5$. A simple comparison among the curves establishes that the anisotropy influences the Rayleigh speed of the isotropic base model. The influence of the invariants $I_{5}$ and $I_{7}$ on the wave speed of the isotropic base model is stronger than the one given by $I_{4}$ and $I_{6}$ in agreement with the results of Vinh et al [62]. Furthermore, under the circumstances at hand, the influence of $I_{4}$ and $I_{6}$ on the speed of the isotropic base model is not strong in the domain of $\lambda$-values shown in the figure.

For $\lambda_{1}=1.2, \lambda_{2}=1, \lambda_{3}=1 / \lambda_{1}$ and waves propagating along the $x_{1}$-axis, 


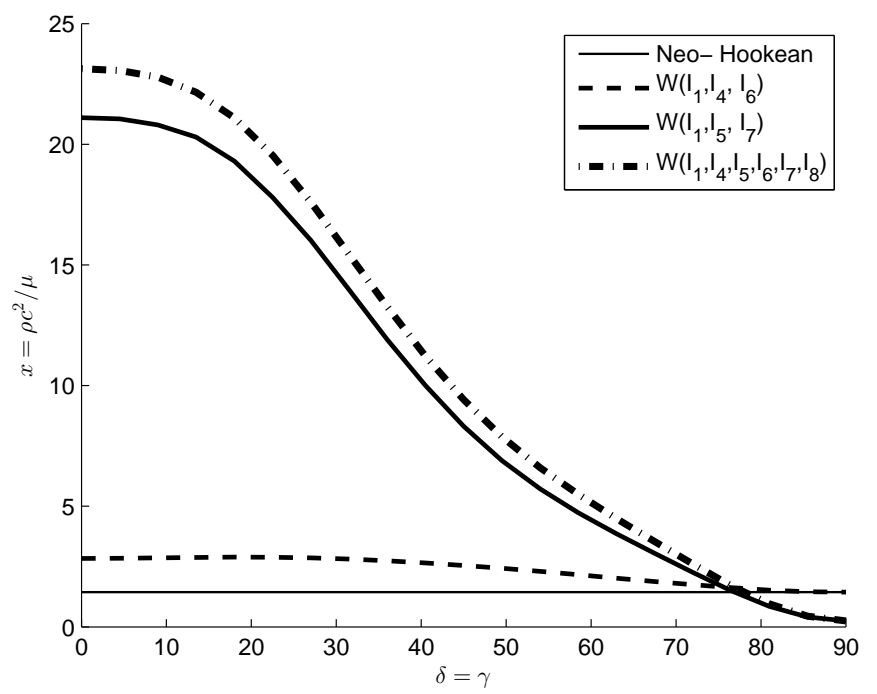

Figure 3.7: Under uniaxial tension along the $X_{1}$-axis with $\lambda_{1}=1.2, \lambda_{2}=1$, $\lambda_{3}=1 / \lambda_{1}$ and waves propagating along the $x_{1}$-axis, the figures shows values of $x=\rho c^{2} / \mu$ vs $\gamma=\delta \in[0, \pi / 2]$, (the angle that each family of fibers makes with the $X_{1}$-direction) as given by (3.30) for (3.33), (3.35) and (3.36), dotted-dashed, dashed and thick solid curves, respectively. The values of the parameters used in the calculations are $k_{1}=k_{3}=0.5 \mu$ and $k_{2}=0.5$. Results for the neo-Hookean model (3.34), the thin solid curve, are also shown for comparison. 
Figure 3.7 shows values of $x=\rho c^{2} / \mu$ vs $\gamma=\delta \in[0, \pi / 2]$ (the angle that each fiber family makes with the $X_{1}$-axis) obtained using (3.30) for (3.33) (dotted-dashed curve), (3.35) (dashed curve), (3.36) (thick solid curve). The parameters used for the calculations are $k_{1}=0.5 \mu$ and $k_{2}=0.5$. The curve associated with the neo-Hookean model (3.34), thin solid one, is horizontal since it is an isotropic model and has the value $x=\rho c^{2} / \mu=$ 1.6227 . 


\section{Surface waves in a layered half-space with initial stress}

\subsection{Introduction}

Guided wave propagation provides an important non-destructive method for assessing material properties and weaknesses in many engineering structures. In the absence of initial stress (residual stress or pre-stress) the classical theory of linear elasticity has been applied successfully in the analysis of such structures. One problem of special interest is the propagation of surface waves in an isotropic linearly elastic layered half-space, and for a treatment of this problem we refer to the classic text [93] for detailed discussion and the papers by [94], [95], [96] and [97].

For a layered half-space of incompressible isotropic elastic material subject to a pure homogeneous finite deformation and an accompanying stress (a so-called pre-stress) the propagation of Rayleigh-type surface waves in a principal plane of the underlying deformation was examined in detail in [98] on the basis of the linearized theory of incremental deformations superimposed on a finite deformation. In the special case of the Murnaghan theory of second-order elasticity [99] also examined the effect of pre-stress on the propagation of surface waves. Surface waves for a half-space with an elastic material boundary without bending stiffness were studied by [100] and generalized to include bending stiffness by [101] following the theory of intrinsic boundary elasticity developed by [102].

For a half-space without a layer subject to a pure homogeneous finite 
deformation the propagation of Rayleigh surface waves was first studied by [103], who, with particular attention to the second-order theory of elasticity, obtained the secular equation for the speed of surface waves first for compressible isotropic materials and then, by specialization, for incompressible materials. Focussing on the incompressible theory for an isotropic material [105] analyzed the propagation of surface waves in a principal plane of a deformed half-space and the limiting case of surface instability for which the wave speed is zero and obtained the secular equation in respect of a general form of strain-energy function. The corresponding problem for a compressible material was treated in [106].

For references to the Barnett-Lothe-Stroh approach to the analysis of surface waves in pre-stressed elastic materials we refer to [107] and [108] in which papers compressible and incompressible materials, respectively, were considered. In contrast to the situation of a half-space subject to finite deformation and a pre-stress associated with it through a constitutive law, for materials with an initial stress parallel to the half-space surface, surface waves were analyzed recently by Shams and Ogden [109] for an incompressible material, and it is an extension of this development to the case of a layered half-space that is the subject of the present chapter. The layer is taken to have a uniform finite thickness and material properties different from those of the half-space, and the initial stress is assumed to be different in the layer and half-space. In the presence of the initial stress (in the reference configuration) the strain-energy function depends on the initial stress as well as on the deformation from the reference configuration.

The basic equations required for the study are presented in Section 4.2 , including development of the constitutive law for an initially stressed elastic material in terms of invariants, as described in Shams and Ogden [109], and its specialization to the case of a plane strain deformation. Section 4.3 provides the incremental equations of motion based on the theory of linearized incremental deformations superimposed on a finite deformation, and expressions for the elasticity tensor of an initially stressed material are given in general form and then explicitly in the case of plane strain for a general form of strain-energy function.

Section 4.4 applies general incremental equations to the expressions that govern two-dimensional motions in the plane of a (pure 
homogeneous) plane strain, a principal plane which is also a principal plane of the considered uniform initial stress. In Section 4.5, these equations are applied to the analysis of surface waves in a homogeneously deformed half-space covered by a layer with a uniform uniaxial initial stress that is parallel to the direction of the wave to obtain the general dispersion equation. The complex form of the dispersion equation derived in Section 4.5 for a general form of strain-energy function is typical for problems involving pre-stressed media, and it is only by careful choice of notation that it is possible to obtain meaningful information from the equation without using an entirely numerical approach. In Section 4.6 the general dispersion equation is solved numerically in respect of a simple form of strain-energy function which extends the basic neo-Hookean material model to include the initial stress. The results are illustrated graphically for several values of the parameters associated with the underlying configuration (initial stress, stretches relative to the reference configuration in layer and half-space, and material parameters).

As a final illustration we exemplify results corresponding to vanishing of the surface wave speed, which corresponds to the emergence of static incremental deformations at critical values of the parameters involved and signals instability of the underlying homogeneous configuration, leading to undulations of layer/half-space structure that decay with depth in the half-space. Such undulations are also referred to as wrinkles, and we refer to the recent paper by [110] for a discussion of wrinkling stability patterns in a graded stiffness half-space.

\subsection{Basic equations}

\subsubsection{Kinematics and stress}

Consider an elastic material occupying some configuration in which there is a known initial (Cauchy) stress $\boldsymbol{\tau}$ which is not specified by a constitutive law. Deformations of the material are measured from this configuration, which is designated as the reference configuration. This is denoted by $\mathcal{B}_{\mathrm{r}}$ and its boundary by $\partial \mathcal{B}_{\mathrm{r}}$. The initial stress satisfies the equilibrium equation $\boldsymbol{\tau}=\mathbf{0}$ in the absence of body forces, and is symmetric in the absence of intrinsic couple stresses, being the divergence operator on $\mathcal{B}_{\mathrm{r}}$. 
If the initial stress is a residual stress, in the sense of Hoger [111], then it also satisfies the zero traction boundary condition $\boldsymbol{\tau} \mathbf{N}=\mathbf{0}$ on $\partial \mathcal{B}_{\mathrm{r}}$, where $\mathbf{N}$ is the unit outward normal to $\partial \mathcal{B}_{\mathrm{r}}$. According to this definition residual stresses are necessarily inhomogeneous, and they have a strong influence on the material response relative to $\mathcal{B}_{\mathrm{r}}$. For references to the literature on the inclusion of residual stress in the constitutive law we refer to [112]. In this paper, however, only initial stresses that are homogeneous will be considered. These also have a significant effect on the material response relative to $\mathcal{B}_{\mathrm{r}}$.

The material is deformed relative to $\mathcal{B}_{\mathrm{r}}$ so that it occupies the deformed configuration $\mathcal{B}$, with boundary $\partial \mathcal{B}$. In standard notation the deformation is described in terms of the vector function $\chi$ according to $\mathbf{x}=\chi(\mathbf{X}), \mathbf{X} \in$ $\mathcal{B}_{\mathrm{r}}$, where $\mathbf{x}$ is the position vector in $\mathcal{B}$ of a material point that had position vector $\mathbf{X}$ in $\mathcal{B}_{\mathrm{r}}$. The deformation gradient tensor $\mathbf{F}$ is defined by $\mathbf{F}=\boldsymbol{\chi}$, where is the gradient operator defined on $\mathcal{B}_{\mathrm{r}}$. We note, in particular, the polar decomposition $\mathbf{F}=\mathbf{V R}$ which will be used subsequently, where the so-called stretch tensor $\mathbf{V}$ is symmetric and positive definite and $\mathbf{R}$ is a proper orthogonal tensor. We shall also make use of the (symmetric) left and right Cauchy-Green deformation tensors, which are given by $\mathbf{B}=\mathbf{F F}^{\mathrm{T}}$ and $\mathbf{C}=\mathbf{F}^{\mathrm{T}} \mathbf{F}$, respectively.

We denote by $\boldsymbol{\sigma}$ the Cauchy stress tensor in the configuration $\mathcal{B}$ and by $\mathbf{S}$ the associated nominal stress tensor relative to $\mathcal{B}_{\mathrm{r}}$, which is given by $\mathbf{S}=J \mathbf{F}^{-1} \boldsymbol{\sigma}$, where $J=\operatorname{det} \mathbf{F}$. We assume that there are no couple stresses, so that $\boldsymbol{\sigma}$ is symmetric. In general, however, the nominal stress tensor is not symmetric, but it follows from the symmetry of $\boldsymbol{\sigma}$ that $\mathbf{F S}=\mathbf{S}^{\mathrm{T}} \mathbf{F}^{\mathrm{T}}$. Body forces are not considered in this paper, so the equilibrium equations to be satisfied by $\boldsymbol{\sigma}$ and $\mathbf{S}$ are $\div \boldsymbol{\sigma}=\mathbf{0}$ and $\mathbf{S}=\mathbf{0}$, respectively, div being the divergence operator on $\mathcal{B}$.

\subsubsection{The strain-energy function}

In the presence of an initial stress $\boldsymbol{\tau}$ the material response relative to $\mathcal{B}_{\mathrm{r}}$ is strongly influenced by $\boldsymbol{\tau}$, and this is reflected in inclusion of $\boldsymbol{\tau}$ in the constitutive law. It can be regarded as a form of structure tensor similar to, but more general than, the structure tensor associated with a preferred direction in $\mathcal{B}_{\mathrm{r}}$. In the present work we consider the material properties to 
be characterized by a strain-energy function $W$, which is defined per unit volume in $\mathcal{B}_{\mathrm{r}}$. In the absence of initial stress $W$ depends on the deformation gradient $\mathbf{F}$, but here it depends also on $\boldsymbol{\tau}$ and we write $W=W(\mathbf{F}, \boldsymbol{\tau})$. For incompressible materials, on which we focus in this paper, the constraint $J \equiv \operatorname{det} \mathbf{F}=1$ must be satisfied for all deformations, and the nominal and Cauchy stress tensors are given by

$$
\mathbf{S}=\frac{\partial W}{\partial \mathbf{F}}(\mathbf{F}, \boldsymbol{\tau})-p \mathbf{F}^{-1}, \quad \boldsymbol{\sigma}=\mathbf{F S}=\mathbf{F} \frac{\partial W}{\partial \mathbf{F}}(\mathbf{F}, \boldsymbol{\tau})-p \mathbf{I},
$$

where $p$ is a Lagrange multiplier associated with the constraint and $\mathbf{I}$ is the identity tensor in $\mathcal{B}$.

\subsubsection{Invariant formulation}

For full details of the constitutive formulation based on invariants, we refer to [113] and [109]. Here we provide a summary of the equations that are needed in the following sections. Since the material is considered to be incompressible there are only two independent invariants of $\mathbf{C}$. We take these to be the standard invariants $I_{1}$ and $I_{2}$ defined by

$$
I_{1}=(\mathbf{C}), \quad I_{2}=\frac{1}{2}\left(I_{1}^{2}-\left(\mathbf{C}^{2}\right)\right)
$$

For $\boldsymbol{\tau}$ three invariants are required in general. These are independent of $\mathbf{C}$ and it is convenient to collect these together as $I_{4}$ according to

$$
I_{4} \equiv\left\{I_{41}, I_{42}, I_{43}\right\}, \quad I_{41}=\boldsymbol{\tau}, \quad I_{42}=\left(\boldsymbol{\tau}^{2}\right), \quad I_{43}=\left(\boldsymbol{\tau}^{3}\right)
$$

The set of invariants is completed by four independent invariants that depend on both $\mathbf{C}$ and $\boldsymbol{\tau}$, which we define by

$$
I_{5}=(\mathbf{C} \boldsymbol{\tau}), \quad I_{6}=\left(\mathbf{C}^{2} \boldsymbol{\tau}\right), \quad I_{7}=\left(\mathbf{C} \boldsymbol{\tau}^{2}\right), \quad I_{8}=\left(\mathbf{C}^{2} \boldsymbol{\tau}^{2}\right)
$$

Note that in the reference configuration (4.2) and (4.4) reduce to

$$
I_{1}=I_{2}=3, \quad I_{5}=I_{6}=\boldsymbol{\tau}, \quad I_{7}=I_{8}=\left(\boldsymbol{\tau}^{2}\right) .
$$

With $W$ regarded as a function of $I_{1}, I_{2}, I_{4}, I_{5}, I_{6}, I_{7}, I_{8}$ the Cauchy stress 
tensor given by $(4.1)_{2}$ can be expanded out as

$$
\begin{aligned}
\boldsymbol{\sigma}=2 W_{1} \mathbf{B}+2 W_{2}\left(I_{1} \mathbf{B}-\mathbf{B}^{2}\right)+ & 2 W_{5} \boldsymbol{\Sigma}+2 W_{6}(\boldsymbol{\Sigma} \mathbf{B}+\mathbf{B} \boldsymbol{\Sigma}) \\
& +2 W_{7} \boldsymbol{\Xi}+2 W_{8}(\boldsymbol{\Xi B}+\mathbf{B} \boldsymbol{\Xi})-p \mathbf{I}(4.6)
\end{aligned}
$$

where $W_{r}=\partial W / \partial I_{r}, r \in\{1,2,5,6,7,8\}, \boldsymbol{\Sigma}=\mathbf{F} \boldsymbol{\tau} \mathbf{F}^{\mathrm{T}}=\mathbf{V R} \boldsymbol{\tau} \mathbf{R}^{\mathrm{T}} \mathbf{V}$ and $\boldsymbol{\Xi}=\mathbf{F} \boldsymbol{\tau}^{2} \mathbf{F}^{\mathrm{T}}=\mathbf{V R} \boldsymbol{\tau}^{2} \mathbf{R}^{\mathrm{T}} \mathbf{V}$

In the reference configuration, equation (4.6) reduces to

$$
\boldsymbol{\tau}=\left(2 W_{1}+4 W_{2}-p^{(\mathrm{r})}\right) \mathbf{I}_{\mathrm{r}}+2\left(W_{5}+2 W_{6}\right) \boldsymbol{\tau}+2\left(W_{7}+2 W_{8}\right) \boldsymbol{\tau}^{2},
$$

where $\mathbf{I}_{\mathrm{r}}$ is the identity tensor in $\mathcal{B}_{\mathrm{r}}, p^{(\mathrm{r})}$ is the value of $p$ in $\mathcal{B}_{\mathrm{r}}$, and all the derivatives of $W$ are evaluated in $\mathcal{B}_{\mathrm{r}}$, where the invariants are given by (4.5). Following Shams et al. [113], but in a slightly different notation, we therefore deduce that

$2 W_{1}+4 W_{2}-p^{(\mathrm{r})}=0, \quad 2\left(W_{5}+2 W_{6}\right)=1, \quad 2\left(W_{7}+2 W_{8}\right)=0 \quad$ in $\mathcal{B}_{\mathrm{r}}(4.8)$

Specializations of these restrictions will be used later.

Suppose that $\mathbf{F}$ now corresponds to a pure homogeneous strain defined by

$$
x_{1}=\lambda_{1} X_{1}, \quad x_{2}=\lambda_{2} X_{2}, \quad x_{3}=\lambda_{3} X_{3},
$$

where $\left(X_{1}, X_{2}, X_{3}\right)$ and $\left(x_{1}, x_{2}, x_{3}\right)$ are Cartesian coordinates in $\mathcal{B}_{\mathrm{r}}$ and $\mathcal{B}$, respectively, and $\lambda_{1}, \lambda_{2}, \lambda_{3}$ are the (uniform) principal stretches. By incompressibility, $\lambda_{1} \lambda_{2} \lambda_{3}=1$. Let $\tau_{i j}, i, j \in\{1,2,3\}$, denote the components of $\boldsymbol{\tau}$ for the considered deformation. Then, referred to the principal axes of the left Cauchy-Green tensor B, which coincide with the Cartesian axes for the pure homogeneous strain, $\Sigma_{i j}=\lambda_{i} \lambda_{j} \tau_{i j}$ and $\Xi_{i j}=\lambda_{i} \lambda_{j}\left(\lambda_{i}^{2}+\lambda_{j}^{2}\right) \sum_{k=1}^{3} \tau_{i k} \tau_{j k}$. The component form of equation (4.6) is then given by

$$
\begin{aligned}
\sigma_{i j}=2 W_{1} \lambda_{i}^{2} \delta_{i j} & +2 W_{2}\left(I_{1}-\lambda_{i}^{2}\right) \lambda_{i}^{2} \delta_{i j}+2\left[W_{5}+W_{6}\left(\lambda_{i}^{2}+\lambda_{j}^{2}\right)\right] \lambda_{i} \lambda_{j} \tau_{i j} \\
& +2\left[W_{7}+\left(\lambda_{i}^{2}+\lambda_{j}^{2}\right) W_{8}\right] \lambda_{i} \lambda_{j} \sum_{k=1}^{3} \tau_{i k} \tau_{j k}-p \delta_{i j} .
\end{aligned}
$$




\subsubsection{Plane strain specialization}

Subsequently, we shall specialize to plane strain (in the 1,2 plane with in-plane principal stretches $\lambda_{1}, \lambda_{2}$ and $\lambda_{3}=1$ ) and with the initial stress confined to this plane, i.e. with $\tau_{i 3}=0$ for $i=1,2,3$. Then, in addition to the standard plane-strain connection $I_{2}=I_{1}$, the connections

$$
\begin{aligned}
& I_{6}=\left(I_{1}-1\right) I_{5}-\left(\tau_{11}+\tau_{22}\right), \\
& I_{7}=\left(\tau_{11}+\tau_{22}\right) I_{5}-\left(\tau_{11} \tau_{22}-\tau_{12}^{2}\right)\left(I_{1}-1\right), \\
& I_{8}=\left(I_{1}-1\right) I_{7}-\left(\tau_{11}^{2}+\tau_{22}^{2}+2 \tau_{12}^{2}\right)
\end{aligned}
$$

can be established. Thus, only two independent invariants that depend on the deformation remain, and we take these to be $I_{1}$ and $I_{5}$. We now write the energy function restricted to plane strain as $\hat{W}\left(I_{1}, I_{5}\right)$ and leave implicit the dependence on the invariants of $\boldsymbol{\tau}$ that do not depend on the deformation.

The in-plane Cauchy stress then takes on the simple form

$$
\boldsymbol{\sigma}=2 \hat{W}_{1} \mathbf{B}+2 \hat{W}_{5} \boldsymbol{\Sigma}-\hat{p} \mathbf{I}
$$

wherein all the tensors are two dimensional (in the 1,2 plane) and $\mathbf{B}$ satisfies the two-dimensional Cayley-Hamilton theorem $\mathbf{B}^{2}-\left(I_{1}-1\right) \mathbf{B}+\mathbf{I}=\mathbf{O}$, the zero tensor, remembering that we are considering incompressibility. Note that $\hat{p}$ is different from the $p$ in (4.6). The conditions (4.8) reduce to

$$
2 \hat{W}_{1}-\hat{p}^{(\mathrm{r})}=0, \quad 2 \hat{W}_{5}=1 .
$$

\subsection{Incremental equations}

In terms of the nominal stress tensor $\mathbf{S}$ the equilibrium equation $\mathbf{S}=\mathbf{0}$ is now written in Cartesian component form as

$$
\mathcal{A}_{\alpha i \beta j} \frac{\partial^{2} x_{j}}{\partial X_{\alpha} \partial X_{\beta}}-\frac{\partial p}{\partial x_{i}}=0
$$


where $\mathcal{A}_{\alpha i \beta j}$ are the components of the elasticity tensor $\mathcal{A}=\mathcal{A}(\mathbf{F}, \boldsymbol{\tau})$. The tensor and component forms are defined by

$$
\mathcal{A}=\frac{\partial^{2} W}{\partial \mathbf{F} \partial \mathbf{F}}, \quad \mathcal{A}_{\alpha i \beta j}=\frac{\partial^{2} W}{\partial F_{i \alpha} \partial F_{j \beta}}
$$

with Greek and Roman indices relating to $\mathcal{B}_{\mathrm{r}}$ and $\mathcal{B}$, respectively.

We now consider a small incremental deformation superimposed on the finite deformation $\mathbf{x}=\chi(\mathbf{X})$. Let this be denoted by $\dot{\mathbf{x}}=\dot{\chi}(\mathbf{X}, t)$ and its gradient by $\dot{\mathbf{x}} \equiv \dot{\mathbf{F}}$. Here and in the following a superposed dot indicates an increment in the considered quantity.

Based on the nominal stress the linearized incremental constitutive equation and the corresponding incremental incompressibility condition are

$$
\dot{\mathbf{S}}=\mathcal{A} \dot{\mathbf{F}}-\dot{p} \mathbf{F}^{-1}+p \mathbf{F}^{-1} \dot{\mathbf{F}} \mathbf{F}^{-1}, \quad\left(\dot{\mathbf{F}} \mathbf{F}^{-1}\right)=0 .
$$

where $\dot{p}$ is the linearized incremental form of $p$.

The incremental equation of motion for an initial homogeneous deformation (with $\mathcal{A}$ and $p$ constants) is then

$$
\dot{\mathbf{S}}=(\mathcal{A} \dot{\mathbf{F}})-\mathbf{F}^{-\mathrm{T}} \dot{p}=\rho \dot{\mathbf{x}}_{, t t},
$$

where a subscript $t$ following a comma indicates the material time derivative and $\rho$ is the mass density of the material. In components this becomes

$$
\mathcal{A}_{\alpha i \beta j} \frac{\partial^{2} \dot{x}_{j}}{\partial X_{\alpha} \partial X_{\beta}}-\frac{\partial \dot{p}}{\partial x_{i}}=\rho \dot{x}_{i, t t}
$$

Also required is the incremental form of the symmetry condition FS = $\mathbf{S}^{\mathrm{T}} \mathbf{F}^{\mathrm{T}}$, i.e.

$$
\mathbf{F} \dot{\mathbf{S}}+\dot{\mathbf{F}} \mathbf{S}=\dot{\mathbf{S}}^{\mathrm{T}} \mathbf{F}^{\mathrm{T}}+\mathbf{S}^{\mathrm{T}} \dot{\mathbf{F}}^{\mathrm{T}}
$$

Following [113] and [109] it is convenient to update the reference configuration so that it coincides with the configuration corresponding to the finite homogeneous deformation with all incremental quantities treated as functions of $\mathbf{x}$ and $t$ instead of $\mathbf{X}$ and $t$. The incremental deformation (displacement) is denoted $\mathbf{u}$ and defined by $\mathbf{u}(\mathbf{x}, t)=\dot{\chi}\left(\chi^{-1}(\mathbf{x}), t\right)$, and all other updated incremental quantities are identified by a zero subscript. In particular, we have $\dot{\mathbf{F}}_{0}=\dot{\mathbf{F}} \mathbf{F}^{-1}=\mathbf{u}$ and 
$\dot{\mathbf{S}}_{0}=\mathbf{F} \dot{\mathbf{S}}$, where is the gradient operator in $\mathcal{B}$, while $\mathcal{A}_{0}$ denotes the updated form of $\mathcal{A}$. In component form we have the connection $\mathcal{A}_{0 p i q j}=F_{p \alpha} F_{q \beta} \mathcal{A}_{\alpha i \beta j}$ (see [82]).

The updated forms of the incremental equation of motion and incompressibility condition are then, in component form,

$$
\mathcal{A}_{0 p i q j} u_{j, p q}-\dot{p}_{, i}=\rho u_{i, t t}, \quad u_{p, p}=0
$$

in which the notations $u_{i, j}=\partial u_{i} / \partial x_{j}, u_{i, j k}=\partial^{2} u_{i} / \partial x_{j} \partial x_{k}$ have been adopted.

The updated form of equation (4.21) yields

$$
\mathcal{A}_{0 i j k l}+\delta_{i l}\left(\sigma_{j k}+p \delta_{j k}\right)=\mathcal{A}_{0 j i k l}+\delta_{j l}\left(\sigma_{i k}+p \delta_{i k}\right),
$$

as given in [113].

At this point we record the strong ellipticity condition on the coefficients $\mathcal{A}_{0 p i q j}$, which states that

$$
\mathcal{A}_{0 p i q j} n_{p} n_{q} m_{i} m_{j}>0
$$

for all non-zero $\mathbf{m}, \mathbf{n}$ such that $\mathbf{m} \cdot \mathbf{n}=0$ (this orthogonality follows from incompressibility), $m_{i}$ and $n_{i}, i=1,2,3$, being the components of $\mathbf{m}$ and $\mathbf{n}$, respectively. In terms of the acoustic tensor $\mathbf{Q}(\mathbf{n})$ defined in component form by $Q_{i j}=\mathcal{A}_{0 p i q j} n_{p} n_{q}$, strong ellipticity ensures that $[\mathbf{Q}(\mathbf{n}) \mathbf{m}] \cdot \mathbf{m}>0$ subject to the stated restrictions on $\mathbf{m}$ and $\mathbf{n}$.

The updated elasticity tensor can be expanded in its component form as

$$
\mathcal{A}_{0 p i q j}=\sum_{r \in \mathcal{I}} W_{r} F_{p \alpha} F_{q \beta} \frac{\partial^{2} I_{r}}{\partial F_{i \alpha} \partial F_{j \beta}}+\sum_{r, s \in \mathcal{I}} W_{r s} F_{p \alpha} F_{q \beta} \frac{\partial I_{r}}{\partial F_{i \alpha}} \frac{\partial I_{s}}{\partial F_{j \beta}}
$$

where $W_{r s}=\partial^{2} W / \partial I_{r} \partial I_{s}$ and $\mathcal{I}$ is the index set $\{1,2,5,6,7,8\}$. Expressions for the derivatives of the invariants which appear in (4.25) and the resulting lengthy expression for $\mathcal{A}_{0 p i q j}$ are given in Shams et al. [113] and are not repeated here. We need only their plane strain specializations, which will be provided in the following. 


\subsubsection{Plane strain case}

Considerable simplification arises in the plane strain specialization considered in Section 4.2.4, for then equation (4.25) applies with the reduced index set $\mathcal{I}=\{1,5\}$. Then the only derivatives of the invariants required are simply

$\frac{\partial I_{1}}{\partial F_{i \alpha}}=2 F_{i \alpha}, \quad \frac{\partial I_{5}}{\partial F_{i \alpha}}=2 \tau_{\alpha \beta} F_{i \beta}, \quad \frac{\partial^{2} I_{1}}{\partial F_{i \alpha} \partial F_{j \beta}}=2 \delta_{\alpha \beta} \delta_{i j}, \quad \frac{\partial^{2} I_{5}}{\partial F_{i \alpha} \partial F_{j \beta}}=2 \tau_{\alpha \beta} \delta_{i j}$.

From (4.25) we then obtain

$$
\begin{aligned}
\mathcal{A}_{0 p i q j} & =2 \hat{W}_{1} B_{p q} \delta_{i j}+2 \hat{W}_{5} \Sigma_{p q} \delta_{i j}+4 \hat{W}_{11} B_{p i} B_{q j} \\
& +4 \hat{W}_{15}\left(B_{p i} \Sigma_{q j}+B_{q j} \Sigma_{p i}\right)+4 \hat{W}_{55} \Sigma_{p i} \Sigma_{q j}
\end{aligned}
$$

with $p, i, q, j$ taking values 1 and 2 .

When specialized to the reference configuration $\mathcal{A}_{0 p i q j}$ is denoted $\mathcal{C}_{\text {piqj }}$, which is given by

$\mathcal{C}_{p i q j}=2 \hat{W}_{1} \delta_{p q} \delta_{i j}+\tau_{p q} \delta_{i j}+4 \hat{W}_{11} \delta_{p i} \delta_{q j}+4 \hat{W}_{15}\left(\delta_{p i} \tau_{q j}+\tau_{p i} \delta_{q j}\right)+4 \hat{W}_{55} \tau_{p i} \tau_{q j}$,

wherein $\hat{W}_{1}, \hat{W}_{11}, \hat{W}_{15}$ and $\hat{W}_{55}$ are evaluated for $I_{1}=3$ and $I_{5}=\tau_{11}+\tau_{22}$ and we have used $(4.15)_{2}$. 


\subsection{Plane incremental motions}

We now illustrate the general theory by specializing the underlying configuration to one consisting of a pure homogeneous strain and focus attention on incremental motions in the $\left(x_{1}, x_{2}\right)$ principal plane, so that the incremental displacement $\mathbf{u}$ has components

$$
u_{1}\left(x_{1}, x_{2}, t\right), \quad u_{2}\left(x_{1}, x_{2}, t\right), \quad u_{3}=0
$$

We also take the initial stress to be uniform and confined to the $\left(x_{1}, x_{2}\right)$ plane, so that $\tau_{i 3}=0, i=1,2,3$. Moreover, the incremental incompressibility condition $(4.22)_{2}$ allows the components $u_{1}$ and $u_{2}$ to be expressed in the form

$$
u_{1}=\psi_{, 2}, \quad u_{2}=-\psi_{, 1},
$$

where $\psi=\psi\left(x_{1}, x_{2}, t\right)$ is a scalar function. Elimination of $\dot{p}$ from the two resulting non-trivial components of the incremental equation of motion $(4.22)_{1}$, as detailed in [109], leads to an equation for $\psi$, namely

$$
\alpha \psi_{, 1111}+2 \delta \psi_{, 1112}+2 \beta \psi_{, 1122}+2 \varepsilon \psi_{, 1222}+\gamma \psi_{, 2222}=\rho\left(\psi_{, 11 t t}+\psi_{, 22 t t}\right)(4.3
$$

in which the (constant) coefficients are defined by

$$
\begin{aligned}
& \alpha=\mathcal{A}_{01212}, \quad 2 \beta=\mathcal{A}_{01111}+\mathcal{A}_{02222}-2 \mathcal{A}_{01122}-2 \mathcal{A}_{02112}, \quad \gamma=\mathcal{A}_{02121} \\
& \delta=\mathcal{A}_{01222}-\mathcal{A}_{01112}, \quad \varepsilon=\mathcal{A}_{01121}-\mathcal{A}_{02122}
\end{aligned}
$$

Given that $\tau_{i 3}=0, i=1,2,3$, we now assume additionally that $\tau_{12}=0$. It follows that $\Sigma_{12}=0$ and $\delta=\varepsilon=0$, and from (4.27) that the coefficients $\alpha, \beta$ and $\gamma$ are given by

$$
\begin{aligned}
\alpha & =2 \hat{W}_{1} \lambda_{1}^{2}+2 \hat{W}_{5} \Sigma_{11}, \quad \gamma=2 \hat{W}_{1} \lambda_{2}^{2}+2 \hat{W}_{5} \Sigma_{22}, \\
2 \beta & =\alpha+\gamma+4 \hat{W}_{11}\left(\lambda_{1}^{2}-\lambda_{2}^{2}\right)^{2} \\
& +8 \hat{W}_{15}\left(\lambda_{1}^{2}-\lambda_{2}^{2}\right)\left(\Sigma_{11}-\Sigma_{22}\right)+4 \hat{W}_{55}\left(\Sigma_{11}-\Sigma_{22}\right)^{2} .
\end{aligned}
$$


In the reference configuration these reduce to

$$
\begin{aligned}
& \alpha=2 \hat{W}_{1}+2 \hat{W}_{5} \tau_{11}, \quad \gamma=2 \hat{W}_{1}+2 \hat{W}_{5} \tau_{22}, \\
& \beta=2 \hat{W}_{1}+\hat{W}_{5}\left(\tau_{11}+\tau_{22}\right)+2 \hat{W}_{55}\left(\tau_{11}-\tau_{22}\right)^{2},
\end{aligned}
$$

with $2 \hat{W}_{5}=1$.

For the considered plane strain the strong ellipticity condition (4.24) specializes to

$$
\alpha n_{1}^{4}+2 \beta n_{1}^{2} n_{2}^{2}+\gamma n_{2}^{4}>0, \quad\left(n_{1}, n_{2}\right) \neq(0,0)
$$

where $\mathbf{m}=\left(-n_{2}, n_{1}, 0\right), \mathbf{n}=\left(n_{1}, n_{2}, 0\right)$. With different values of $\alpha, \beta$ and $\gamma$ necessary and sufficient conditions for (4.36) to hold were given by [105] as

$$
\alpha>0, \quad \gamma>0, \quad \beta>-\sqrt{\alpha \gamma}
$$

\subsection{Surface waves in a layered half-space}

In this section we consider Rayleigh-type elastic surface waves guided by a layer bonded to the surface of a half-space, the layer being of a different material than that of the half-space. Let us consider an initially stressed half-space that is subjected to a pure homogeneous strain with principal stretches $\lambda_{1}, \lambda_{2}, \lambda_{3}$ so that the deformed half-space is defined by $x_{2}<0$ with boundary $x_{2}=0$ and we focus attention on the $\left(x_{1}, x_{2}\right)$ principal plane. The initial stress is also taken to be uniform, and we have already assumed that $\tau_{i j}=0, i \neq j$. The layer has uniform thickness $h$ in the deformed configuration and is defined by $0 \leq x_{2} \leq h$. The (planar) invariants for the material of half space are $I_{1}, I_{5}$, while the notations $I_{1}^{*}$ and $I_{5}^{*}$ are used for the layer. The (plane strain) elasticity tensor for the half-space is given by (4.27) and the corresponding elasticity tensor for the layer has a similar form but with $\hat{W}, \mathbf{B}$ and $\boldsymbol{\Sigma}$ replaced by $\hat{W}^{*}, \mathbf{B}^{*}$ and $\boldsymbol{\Sigma}^{*}$.

On specializing equation (4.14) we then obtain the only non-zero Cauchy stress components as

$$
\sigma_{i i}=2 \hat{W}_{1} \lambda_{i}^{2}+2 \hat{W}_{5} \lambda_{i}^{2} \tau_{i i}-p, \quad i=1,2,3 \text { (no summation) }
$$


for the half-space, and similarly for the layer:

$$
\sigma_{i i}^{*}=2 \hat{W}_{1}^{*} \lambda_{i}^{* 2}+2 \hat{W}_{5}^{*} \lambda_{i}^{* 2} \tau_{i i}^{*}-p^{*}, \quad i=1,2,3 \text { (no summation). }
$$

Now consider plane incremental motions within the half-space and layer with incremental displacements $\mathbf{u}$ and $\mathbf{u}^{*}$, respectively, having components

$$
u_{1}\left(x_{1}, x_{2}, t\right), \quad u_{2}\left(x_{1}, x_{2}, t\right), \quad u_{1}^{*}\left(x_{1}, x_{2}, t\right), \quad u_{2}^{*}\left(x_{1}, x_{2}, t\right),
$$

with $u_{3}=u_{3}^{*}=0$.

We assume that the boundary $x_{2}=h$ of the layer is free of incremental traction, so that

$$
\dot{S}_{021}^{*}=0, \quad \dot{S}_{022}^{*}=0 \quad \text { on } \quad x_{2}=h
$$

We also consider both the displacement and the incremental traction to be continuous at the interface $x_{2}=0$, so that

$$
u_{1}=u_{1}^{*}, \quad u_{2}=u_{2}^{*}, \quad \dot{S}_{021}^{*}=\dot{S}_{021}, \quad \dot{S}_{022}^{*}=\dot{S}_{022} \quad \text { on } \quad x_{2}=0
$$

The non-trivial components of the incremental traction per unit area of the surface and layer, respectively, are $\dot{S}_{02 i}$ and $\dot{S}_{02 i}^{*}, i=1,2$, which are given by

$$
\begin{gathered}
\dot{S}_{02 i}=\mathcal{A}_{02 i l k} u_{k, l}+p u_{2, i}-\dot{p} \delta_{2 i}, \quad i=1,2, \\
\dot{S}_{02 i}^{*}=\mathcal{A}_{02 i l k}^{*} u_{k, l}^{*}+p^{*} u_{2, i}^{*}-\dot{p}^{*} \delta_{2 i}, \quad i=1,2 .
\end{gathered}
$$

By differentiating (4.43) and (4.44) for $i=2$ with respect to $x_{1}$ and eliminating $\dot{p}_{, 1}$ using the first component of the equation of motion (as in [109] for the half-space problem), and similarly for $\dot{p}_{, 1}^{*}$, the incremental traction continuity conditions $(4.42)_{3,4}$ are expressed in terms of $\psi$ and its 
counterpart $\psi^{*}$ for the layer as

$$
\begin{aligned}
& \left(\sigma_{22}-\gamma\right) \psi_{, 11}+\gamma \psi_{, 22}=\left(\sigma_{22}^{*}-\gamma^{*}\right) \psi_{, 11}^{*}+\gamma^{*} \psi_{, 22}^{*}, \\
& \rho \psi_{, 2 t t}-\left(2 \beta+\gamma-\sigma_{22}\right) \psi_{, 112}-\gamma \psi_{, 222} \\
& =\rho^{*} \psi_{, 2 t t}^{*}-\left(2 \beta^{*}+\gamma^{*}-\sigma_{22}^{*}\right) \psi_{, 112}^{*}-\gamma^{*} \psi_{, 222}^{*}
\end{aligned}
$$

on $x_{2}=0$, the latter corresponding to $\dot{S}_{022,1}=\dot{S}_{022,1}^{*}$. Note that by continuity of the underlying configuration $\sigma_{22}^{*}=\sigma_{22}$. The zero incremental traction boundary conditions on $x_{2}=h$ are

$$
\begin{aligned}
\dot{S}_{021}^{*} & =\left(\sigma_{22}^{*}-\gamma^{*}\right) \psi_{, 11}^{*}+\gamma^{*} \psi_{, 22}^{*}=0 \\
\dot{S}_{022,1}^{*} & =\rho^{*} \psi_{, 2 t t}^{*}-\left(2 \beta^{*}+\gamma^{*}-\sigma_{22}^{*}\right) \psi_{, 112}^{*}-\gamma^{*} \psi_{, 222}^{*}=0
\end{aligned}
$$

Here we have used the connection

$$
\mathcal{A}_{0 i j i j}-\mathcal{A}_{0 i j j i}=\sigma_{i i}+p, \quad i \neq j
$$

which can be obtained from (4.23), and the corresponding one for the layer. We now specialize the initial stress so that it has just one non-zero component, namely $\tau_{11}, \tau_{11}^{*}$, in the half-space and layer, respectively. We also assume that there is no traction on the boundary $x_{2}=0$ associated with the underlying configuration, so that $\sigma_{22}=0$ and $\sigma_{22}^{*}=0$.

We consider surface waves propagating along the $x_{1}$ axis, which forms with $x_{2}$ a pair of principal axes of the underlying deformation so that the displacement components are given by (4.40). We take the surface wave to have the form

$$
\psi=A \exp \left[s k x_{2}-\mathrm{i} k\left(x_{1}-c t\right)\right], \quad \psi^{*}=A^{*} \exp \left[s^{*} k x_{2}-\mathrm{i} k\left(x_{1}-c t\right)\right]
$$

in the half-space and layer, respectively, where $A, A^{*}$ are constants, $k$ is the wave number, $c$ is the wave speed, and $s, s^{*}$ are to be determined. Using equation (4.50) in the equation of motion (4.31), we obtain

$$
\gamma s^{4}-\left(2 \beta-\rho c^{2}\right) s^{2}+\left(\alpha-\rho c^{2}\right)=0
$$


and

$$
\gamma^{*} s^{* 4}-\left(2 \beta^{*}-\rho^{*} c^{2}\right) s^{* 2}+\left(\alpha^{*}-\rho^{*} c^{2}\right)=0
$$

for the half-space and layer, respectively.

For the half-space the solutions have to decay as $x_{2} \rightarrow-\infty$, which requires that the relevant solutions of (4.51) for $s$ should have positive real parts. Let $s_{1}$ and $s_{2}$ be those solutions. Since $-s^{*}$ is a solution of (4.52) whenever $s^{*}$ is, let $s_{1}^{*}, s_{2}^{*},-s_{1}^{*}$ and $-s_{2}^{*}$ denote the roots. The general solutions for $\psi$ and $\psi^{*}$ of the considered type may then be written in the form

$$
\psi=\left(A_{1} \mathrm{e}^{s_{1} k x_{2}}+A_{2} \mathrm{e}^{s_{2} k x_{2}}\right) \exp \left[\mathrm{i} k\left(c t-x_{1}\right)\right]
$$

and

$$
\psi^{*}=\left(A_{1}^{*} \mathrm{e}^{s_{1}^{*} k x_{2}}+A_{2}^{*} \mathrm{e}^{s_{2}^{*} k x_{2}}+A_{3}^{*} \mathrm{e}^{-s_{1}^{*} k x_{2}}+A_{4}^{*} \mathrm{e}^{-s_{2}^{*} k x_{2}}\right) \exp \left[\mathrm{i} k\left(c t-x_{1}\right)\right]
$$

where $A_{i}, i=1,2$, and $A_{i}^{*}, i=1, \ldots, 4$, are constants.

Following the arguments in Dowaikh and Ogden [105] and Ogden and Sotiropoulos [98] we may deduce that there is an upper bound on the wave speed according to

$$
0 \leq \rho c^{2} \leq \rho c_{\mathrm{L}}^{2}= \begin{cases}\alpha & \text { if } 2 \beta \geq \alpha \\ 2 \beta-2 \gamma+2 \sqrt{\gamma} \sqrt{\alpha+\gamma-2 \beta} & \text { if } 2 \beta \leq \alpha\end{cases}
$$

where $c_{\mathrm{L}}(>0)$ is the limiting speed. The limiting value $c_{\mathrm{L}}$ for the case when $2 \beta \geq \alpha$ is the speed of a plane shear wave propagating in the $x_{1}$-direction with displacement in the $x_{2}$-direction in an unbounded body subjected to the same homogeneous pure strain and initial stress. It does not correspond to a surface wave, and it is straightforward to show that $2 \beta-2 \gamma+2 \sqrt{\gamma} \sqrt{\alpha+\gamma-2 \beta} \leq \alpha$, with equality if $2 \beta=\alpha$.

At this point it is convenient to define the notation

$$
\eta=\left[\left(\alpha-\rho c^{2}\right) / \gamma\right]^{1 / 2}, \quad \eta^{2}=s_{1}^{2} s_{2}^{2}=\left(\alpha-\rho c^{2}\right) / \gamma
$$

In order to qualify as a surface wave in the half-space $s_{1}^{2} s_{2}^{2}$ must be positive and hence, without loss of generality, we may take $\eta=s_{1} s_{2}>0$, so that 
$s_{1}$ and $s_{2}$ must either both be real and positive or be complex conjugates. If they are real

$$
\left(s_{1}+s_{2}\right)^{2}=\eta^{2}+2 \eta+2 \bar{\beta}-\bar{\alpha}>0, \quad\left(s_{1}-s_{2}\right)^{2}=\eta^{2}-2 \eta+2 \bar{\beta}-\bar{\alpha}>0
$$

while if they are complex conjugates

$$
\left(s_{1}+s_{2}\right)^{2}=\eta^{2}+2 \eta+2 \bar{\beta}-\bar{\alpha}>0, \quad\left(s_{1}-s_{2}\right)^{2}=\eta^{2}-2 \eta+2 \bar{\beta}-\bar{\alpha}<0,
$$

within which we have defined the notation

$$
\bar{\alpha}=\alpha / \gamma, \quad \bar{\beta}=\beta / \gamma
$$

The counterpart of (4.55) in respect of $\eta$ is then

$$
\sqrt{\bar{\alpha}} \geq \eta \geq \eta_{\mathrm{L}}=\left\{\begin{array}{l}
0 \quad \text { if } \quad 2 \beta \geq \alpha \\
(1+\bar{\alpha}-2 \bar{\beta})^{1 / 2}-1 \quad \text { if } \quad 2 \beta \leq \alpha
\end{array}\right.
$$

wherein $\eta_{\mathrm{L}}$, the lower limiting value of $\eta$, is defined.

Similarly, we define

$$
\eta^{*}=\left[\left(\alpha^{*}-\rho^{*} c^{2}\right) / \gamma^{*}\right]^{1 / 2}, \quad \eta^{* 2}=s_{1}^{* 2} s_{2}^{* 2}=\left(\alpha^{*}-\rho^{*} c^{2}\right) / \gamma^{*},
$$

but we note that in contrast to $\eta^{2}, \eta^{* 2}$ may be either positive or negative, and hence $\eta^{*}$ may be real or pure imaginary. We now consider these two possibilities separately, with the notation

$$
\bar{\alpha}^{*}=\alpha^{*} / \gamma^{*}, \quad \bar{\beta}^{*}=\beta^{*} / \gamma^{*}
$$

Case (a): $\eta^{* 2}<0$.

In this case $s_{1}^{* 2}$ and $s_{2}^{* 2}$ cannot be complex conjugates and so must be real and have opposite signs. If

$$
s_{1}^{* 2}+s_{2}^{* 2}<0 \quad \text { then } \rho^{*} c^{2} / \gamma^{*}>\max \left\{\bar{\alpha}^{*}, 2 \bar{\beta}^{*}\right\}
$$

while if

$$
s_{1}^{* 2}+s_{2}^{* 2}>0 \quad \text { then } \quad \bar{\alpha}^{*}<\rho^{*} c^{2} / \gamma^{*}<2 \bar{\beta}^{*} .
$$


On setting $s_{1}^{*} s_{2}^{*}=\eta^{*}$ we also note that

$$
\left(s_{1}^{*}+s_{2}^{*}\right)^{2}=\eta^{* 2}+2 \eta^{*}+2 \bar{\beta}^{*}-\bar{\alpha}^{*} \quad \text { and } \quad\left(s_{1}^{*}-s_{2}^{*}\right)^{2}=\eta^{* 2}-2 \eta^{*}+2 \bar{\beta}^{*}-\bar{\alpha}^{*}
$$

are complex conjugates.

Case (b): $\eta^{* 2}>0$.

Without loss of generality we take $s_{1}^{*} s_{2}^{*}=\eta^{*}>0$. Let us first consider the situation in which $s_{1}^{* 2}$ and $s_{2}^{* 2}$ are real. Then, we have either

$$
s_{1}^{* 2}>0 \text { and } s_{2}^{* 2}>0 \quad \text { with } \rho^{*} c^{2} / \gamma^{*}<\min \left\{\bar{\alpha}^{*}, 2 \bar{\beta}^{*}\right\}
$$

and

$$
\eta^{* 2} \pm 2 \eta^{*}+2 \bar{\beta}^{*}-\bar{\alpha}^{*}>0
$$

or

$$
s_{1}^{* 2}<0 \text { and } s_{2}^{* 2}<0 \quad \text { with } 2 \bar{\beta}^{*}<\rho^{*} c^{2} / \gamma^{*}<\bar{\alpha}^{*}
$$

and

$$
\eta^{* 2} \pm 2 \eta^{*}+2 \bar{\beta}^{*}-\bar{\alpha}^{*}<0 .
$$

On the other hand, if $s_{1}^{* 2}$ and $s_{2}^{* 2}$ are complex conjugates

$$
\eta^{* 2}+2 \eta^{*}+2 \bar{\beta}^{*}-\bar{\alpha}^{*}>0>\eta^{* 2}-2 \eta^{*}+2 \bar{\beta}^{*}-\bar{\alpha}^{*}
$$

with $2 \bar{\beta}^{*}-\rho^{*} c^{2} / \gamma^{*}$ having either sign:

$$
2 \bar{\beta}^{*}<\rho^{*} c^{2} / \gamma^{*}<\bar{\alpha}^{*} \quad \text { or } \quad \rho^{*} c^{2} / \gamma^{*}<\min \left\{\bar{\alpha}^{*}, 2 \bar{\beta}^{*}\right\}
$$

Each of the possibilities in Case (a) and Case (b) arises within the numerical examples illustrated in the following section.

Substituting (4.53) and (4.54) into the boundary conditions (4.41) and (4.42), expressed through (4.45)-(4.48), with use of (4.51) and (4.52) we obtain

$$
\left(A_{1}^{*} \mathrm{e}^{s_{1}^{*} k h}+A_{3}^{*} \mathrm{e}^{-s_{1}^{*} k h}\right)\left(1+s_{1}^{* 2}\right)+\left(A_{2}^{*} \mathrm{e}^{s_{2}^{*} k h}+A_{4}^{*} \mathrm{e}^{-s_{2}^{*} k h}\right)\left(1+s_{2}^{* 2}\right)=0,(4.72)
$$




$$
\begin{gathered}
\left(A_{1}^{*} \mathrm{e}^{s_{1}^{*} k h}-A_{3}^{*} \mathrm{e}^{-s_{1}^{*} k h}\right) s_{1}^{*}\left(1+s_{2}^{* 2}\right) \\
+\left(A_{2}^{*} \mathrm{e}^{s_{2}^{*} k h}-A_{4}^{*} \mathrm{e}^{-s_{2}^{*} k h}\right) s_{2}^{*}\left(1+s_{1}^{* 2}\right)=0, \\
A_{1}+A_{2}-A_{1}^{*}-A_{2}^{*}-A_{3}^{*}-A_{4}^{*}=0, \\
A_{1} s_{1}+A_{2} s_{2}-\left(A_{1}^{*}-A_{3}^{*}\right) s_{1}^{*}-\left(A_{2}^{*}-A_{4}^{*}\right) s_{2}^{*}=0, \\
{\left[A_{1}\left(s_{1}^{2}+1\right)+A_{2}\left(s_{2}^{2}+1\right)\right] \gamma} \\
-\left[\left(A_{1}^{*}+A_{3}^{*}\right)\left(s_{1}^{* 2}+1\right)+\left(A_{2}^{*}+A_{4}^{*}\right)\left(s_{2}^{* 2}+1\right)\right] \gamma^{*}=0, \\
{\left[A_{1} s_{1}\left(1+s_{2}^{2}\right)+A_{2} s_{2}\left(1+s_{1}^{2}\right)\right] \gamma} \\
-\left[\left(A_{1}^{*}-A_{3}^{*}\right) s_{1}^{*}\left(1+s_{2}^{* 2}\right)-\left(A_{2}^{*}-A_{4}^{*}\right) s_{2}^{*}\left(1+s_{1}^{* 2}\right)\right] \gamma^{*}=0 .
\end{gathered}
$$

The set of equations (4.72)-(4.77) can be written in the matrix form

$$
\mathcal{M A}=\mathbf{0}
$$

where $\mathbf{A}=\left(A_{1}, A_{2}, A_{1}^{*}, A_{2}^{*}, A_{3}^{*}, A_{4}^{*}\right)$ and $\mathcal{M}$ is the $6 \times 6$ matrix

$$
\left.\mathcal{M}=\left[\begin{array}{cccccc}
1 & 1 & -1 & -1 & -1 & -1 \\
s_{1} & s_{2} & -s_{1}^{*} & -s_{2}^{*} & s_{1}^{*} & s_{2}^{*} \\
\gamma s_{1} S_{2} & \gamma s_{2} S_{1} & -\gamma^{*} s_{1}^{*} S_{2}^{*} & -\gamma^{*} s_{2}^{*} S_{1}^{*} & \gamma^{*} s_{1}^{*} S_{2}^{*} & \gamma^{*} s_{2}^{*} S_{1}^{*} \\
\gamma S_{1} & \gamma S_{2} & -\gamma^{*} S_{1}^{*} & -\gamma^{*} S_{2}^{*} & -\gamma^{*} S_{1}^{*} & -\gamma^{*} S_{2}^{*} \\
0 & 0 & S_{1}^{*} e^{s_{1}^{*} k h} & S_{2}^{*} e^{s_{2}^{*} k h} & S_{1}^{*} e^{-s_{1}^{*} k h} & S_{2}^{*} e^{-s_{2}^{*} k h} \\
0 & 0 & s_{1}^{*} S_{2}^{*} e^{s_{1}^{*} k h} & s_{2}^{*} S_{1}^{*} e^{s_{2}^{*} k h} & -s_{1}^{*} S_{2}^{*} e^{-s_{1}^{*} k h} & -s_{2}^{*} S_{1}^{*} e^{-s_{2}^{*} k h}
\end{array}\right] 4.78\right)
$$

within which we have used the notation $S_{i}=1+s_{i}^{2}, S_{i}^{*}=1+s_{i}^{* 2}, i=1,2$. For a nontrivial solution, the determinant of $\mathcal{M}$ must vanish. After considerable manipulation it can be shown that $\operatorname{det} \mathcal{M}$ can be written

$$
\operatorname{det} \mathcal{M}=-2\left(s_{1}-s_{2}\right)\left(s_{1}^{*}-s_{2}^{*}\right)^{2}\left(s_{1}^{*}+s_{2}^{*}\right)^{2} \mathcal{N}
$$


where $\mathcal{N}$ has the form

$$
\begin{aligned}
\mathcal{N} & =A\left(\eta, \eta^{*}\right) \frac{\sinh ^{2}\left[\frac{1}{2} k h\left(s_{1}^{*}+s_{2}^{*}\right)\right]}{\left(s_{1}^{*}+s_{2}^{*}\right)^{2}}-A\left(\eta,-\eta^{*}\right) \frac{\sinh ^{2}\left[\frac{1}{2} k h\left(s_{1}^{*}-s_{2}^{*}\right)\right]}{\left(s_{1}^{*}-s_{2}^{*}\right)^{2}} \\
& +B\left(\eta, \eta^{*}\right) \frac{\sinh \left[k h\left(s_{1}^{*}+s_{2}^{*}\right)\right]}{\left(s_{1}^{*}+s_{2}^{*}\right)}-B\left(\eta,-\eta^{*}\right) \frac{\sinh \left[k h\left(s_{1}^{*}-s_{2}^{*}\right)\right]}{\left(s_{1}^{*}-s_{2}^{*}\right)} \\
& +C\left(\eta, \eta^{*}\right)
\end{aligned}
$$

in which, following [98], we have introduced the definitions

$$
\begin{gathered}
A\left(\eta, \eta^{*}\right)=2 f^{*}\left(\eta^{*}\right)\left[\gamma^{2} f(\eta)+\gamma^{* 2} f^{*}\left(\eta^{*}\right)+2 \gamma \gamma^{*}(\eta-1)\left(\eta^{*}-1\right)\right] \\
B\left(\eta, \eta^{*}\right)=f^{*}\left(\eta^{*}\right) \gamma \gamma^{*}\left(\eta+\eta^{*}\right) \eta^{-1 / 2}\left[f(\eta)+(\eta-1)^{2}\right]^{1 / 2} \\
C\left(\eta, \eta^{*}\right)=2 \gamma^{2} \eta^{*} f(\eta)
\end{gathered}
$$

with

$$
f(\eta)=\eta^{3}+\eta^{2}+d \eta-1, \quad f\left(\eta^{*}\right)=\eta^{* 3}+\eta^{* 2}+d^{*} \eta^{*}-1 .
$$

We have also defined

$$
d=2 \bar{\beta}+2-\bar{\alpha}, \quad d^{*}=2 \bar{\beta}^{*}+2-\bar{\alpha}^{*}
$$

Note that

$$
\left(s_{1}+s_{2}\right)^{2}=\eta^{2}+2 \eta+2 \bar{\beta}-\bar{\alpha}=\eta^{-1}\left[f(\eta)+(\eta-1)^{2}\right]>0
$$

and

$$
\left(s_{1}^{*}+s_{2}^{*}\right)^{2}=\eta^{* 2}+2 \eta^{*}+2 \bar{\beta}^{*}-\bar{\alpha}^{*}=\eta^{*-1}\left[f^{*}\left(\eta^{*}\right)+\left(\eta^{*}-1\right)^{2}\right],
$$

the latter being complex in Case (a).

It is straightforward to show that $\mathcal{N}$ is real if $\eta^{* 2}>0$ and pure imaginary if $\eta^{* 2}<0$. The formula (4.79) is the same as one derived in [98] [equation (3.6) therein] apart from slight differences in notation. However, the content is different since the values of the material parameters $\alpha, \beta, \gamma$ and their starred counterparts are different. We focus first on some special cases of $\mathcal{N}=0$ and then consider briefly the other factors in the 
expression for $\operatorname{det} \mathcal{M}$.

First, the limiting case $k h \rightarrow 0$ corresponds to a half-space for which the secular equation

$$
f(\eta)=\eta^{3}+\eta^{2}+d \eta-1=0
$$

has been analyzed in detail in [109].

Second, $k h \rightarrow \infty$ corresponds to the secular equation for interfacial (Stoneley-type) waves along the boundary between two half-spaces, having the equation

$$
\begin{aligned}
\gamma^{* 2} f^{*}\left(\eta^{*}\right) & +\gamma \gamma^{*}\left(\eta+\eta^{*}\right) \eta^{-1 / 2} \eta^{*-1 / 2}\left[f(\eta)+(\eta-1)^{2}\right]^{1 / 2}\left[f^{*}\left(\eta^{*}\right)+\left(\eta^{*}-1\right)^{2}\right]^{1 / 2} \\
& +2 \gamma \gamma^{*}(\eta-1)\left(\eta^{*}-1\right)+\gamma^{2} f(\eta)=0
\end{aligned}
$$

For the case in which the initial stress is a pre-stress associated with a finite deformation this equation was derived by [106], but expressed in different notation.

The third special case corresponds to the absence of the half-space $(\gamma \rightarrow 0)$, and the dispersion equation reduces to

$$
\left[f^{*}\left(\eta^{*}\right)\right]^{2} \frac{\sinh ^{2}\left[\frac{1}{2} k h\left(s_{1}^{*}+s_{2}^{*}\right)\right]}{\left(s_{1}^{*}+s_{2}^{*}\right)^{2}}=\left[f^{*}\left(-\eta^{*}\right)\right]^{2} \frac{\sinh ^{2}\left[\frac{1}{2} k h\left(s_{2}^{*}-s_{1}^{*}\right)\right]}{\left(s_{2}^{*}-s_{1}^{*}\right)^{2}} .
$$

This provides the dispersion equation for Lamb-type waves in a plate with uniform thickness $h$ and of infinite extent in the lateral directions.

The final special case corresponds to $c=0\left(\eta=\sqrt{\bar{\alpha}}, \eta^{*}=\sqrt{\bar{\alpha}^{*}}\right)$, in which case equation (4.79) specializes accordingly and provides a criterion for the existence of quasi-static incremental deformations.

Vanishing of either of the other factors $s_{1}-s_{2}, s_{1}^{*}-s_{2}^{*}$ or $s_{1}^{*}+s_{2}^{*}$ may also lead to solutions of the secular equation, but each such solution that exists is independent of $k h$ and arises as a special case of $\mathcal{N}=0$ for specific ranges of values of $\bar{\alpha}, \bar{\beta}, \bar{\alpha}^{*}$ and $\bar{\beta}^{*}$.

\subsection{Numerical illustrations}

In order to illustrate the solutions of the secular equation $\mathcal{N}=0$ we now select the simple prototype form of strain-energy function that was used 
in Shams et al. [113] and given (in slightly different notation) by

$$
W=\frac{1}{2} \mu\left(I_{1}-3\right)+\frac{1}{4} \mu_{1}\left[I_{5}-(\boldsymbol{\tau})\right]^{2}+\frac{1}{2}\left[I_{5}-(\boldsymbol{\tau})\right],
$$

where $\mu>0$ is a material constant with the dimension of stress and $\mu_{1}$ is a material constant with dimension of stress ${ }^{-1}$. We allow $\mu_{1}$ to be either positive or negative. The first term is the classical neo-Hookean model of rubber elasticity, while the second and third terms introduce the residual stress in a very simple form involving just the invariant $I_{5}$ (and its specialization to the reference configuration) and ensuring that the condition $(4.8)_{2}$ is satisfied. For the plane strain problem considered in the previous section we write $(4.89)$ as $\hat{W}\left(I_{1}, I_{5}\right)$, the underlying deformation corresponding to $\lambda_{1}=\lambda, \lambda_{2}=\lambda^{-1}$ and $\lambda_{3}=1$. In the layer quantities are distinguished by an asterisk.

As we have already assumed the boundary $x_{2}=h$ is free of traction in the underlying configuration. Thus, $\sigma_{22}=0$ and correspondingly $\tau_{22}=0$. The Cauchy stress components are then obtained by specializing (4.14) as

$$
\begin{aligned}
& \sigma_{11}=\mu \lambda^{2}+\lambda^{2} \tau+\mu_{1}\left(\lambda^{2}-1\right) \lambda^{2} \tau^{2}-p, \\
& 0=\sigma_{22}=\mu \lambda^{-2}-p, \quad \sigma_{33}=\mu-p,
\end{aligned}
$$

where $\tau_{11}$ has been written simply as $\tau$. Hence, on elimination of $p$,

$$
\sigma_{11}=\mu\left(\lambda^{2}-\lambda^{-2}\right)+\lambda^{2} \tau+\mu_{1}\left(\lambda^{2}-1\right) \lambda^{2} \tau^{2}, \quad \sigma_{33}=\mu\left(1-\lambda^{-2}\right),
$$

the latter component being required to maintain the plane strain condition. Similarly, for the layer we have

$$
\sigma_{11}^{*}=\mu^{*}\left(\lambda^{* 2}-\lambda^{*-2}\right)+\lambda^{* 2} \tau^{*}+\mu_{1}^{*}\left(\lambda^{* 2}-1\right) \lambda^{* 2} \tau^{* 2}, \quad \sigma_{33}^{*}=\mu^{*}\left(1-\lambda^{*-2}\right) .
$$

At this point a comment on the effect of the term in $\mu_{1}$ on the material response in the half-space is called for. In plane strain tension $(\lambda>1)$, for example, positive $\mu_{1}$ increases the stiffness of the response, while negative $\mu_{1}$ decreases the stiffness, the material softens on extension and the Cauchy stress reaches a maximum. Similarly for the layer. 
For the model (4.89), the material coefficients are given by

$$
\begin{gathered}
\bar{\alpha}=\lambda^{4}\left[1+\bar{\tau}+\bar{\mu}\left(\lambda^{2}-1\right) \bar{\tau}^{2}\right], \quad d=2 \bar{\beta}-\bar{\alpha}+2=3+2 \bar{\mu} \lambda^{6} \bar{\tau}^{2}, \quad \\
\bar{\alpha}^{*}=\lambda^{* 4}\left[1+\bar{\tau}^{*}+\bar{\mu}^{*}\left(\lambda^{* 2}-1\right) \bar{\tau}^{* 2}\right], \quad d^{*}=2 \bar{\beta}^{*}-\bar{\alpha}^{*}+2=3+2 \bar{\mu}^{*} \lambda^{* 6} \bar{\tau}^{* 2} 4.94
\end{gathered}
$$

where we have used $\Sigma_{11}=\lambda^{2} \tau, \Sigma_{11}^{*}=\lambda^{* 2} \tau^{*}$ and introduced the dimensionless parameters $\bar{\tau}=\tau / \mu, \bar{\tau}^{*}=\tau^{*} / \mu^{*}, \bar{\mu}=\mu \mu_{1}$ and $\bar{\mu}^{*}=\mu^{*} \mu_{1}^{*}$.

We next consider the important special case $k h=0$ corresponding to a half-space without a layer that was treated by [109]. For $k h=0$ the secular equation reduces to $f(\eta)=0$, where $f(\eta)$ is given by (4.86), which is the result obtained in [109]. Clearly $f(0)=-1$. Also, it is straightforward to show that $f\left(\eta_{\mathrm{L}}\right)<0$. Hence, the requirement for the existence of a surface wave is $f\left(\bar{\alpha}^{1 / 2}\right)>0$, and this gives

$$
0 \leq \eta_{\mathrm{L}}<\eta<\sqrt{\bar{\alpha}}=\lambda^{2} \sqrt{\epsilon}
$$

and

$$
\xi \equiv \lambda^{6} \epsilon^{3 / 2}+\lambda^{4} \epsilon+\left(3+2 \bar{\mu} \lambda^{6} \bar{\tau}^{2}\right) \lambda^{2} \epsilon^{1 / 2}-1>0,
$$

wherein we have defined $\xi$ and introduced the notation

$$
\epsilon=1+\bar{\tau}+\bar{\mu}\left(\lambda^{2}-1\right) \bar{\tau}^{2}>0
$$

Note, with reference to $(4.37)$, that $f\left(\bar{\alpha}^{1 / 2}\right)>0$ ensures that strong ellipticity holds since

$$
f\left(\bar{\alpha}^{1 / 2}\right)=\bar{\alpha}+2(\bar{\beta}+1) \bar{\alpha}^{1 / 2}-1>0
$$

implies

$$
\left(2 \bar{\beta}+2 \bar{\alpha}^{1 / 2}\right) \bar{\alpha}^{1 / 2}>\left(\bar{\alpha}^{1 / 2}-1\right)^{2} \geq 0 .
$$

As shown in [109] for a half-space, when a surface wave exists it is unique. For the existence of a surface wave when $k h=0$ we require both $\epsilon>\lambda^{-4} \eta_{\mathrm{L}}^{2}$ and $\xi>0$. If $\bar{\mu}>0$ then $\eta_{\mathrm{L}}=0$, but if $\bar{\mu}<0$ then $\eta_{\mathrm{L}}$ is only zero for certain ranges of values of $\lambda$ and $\bar{\tau}$, as discussed in [109]. [Note that there is a typo in equation (6.26) of [109] (1/8 should be $1 / 4)$.] 
Examples of the region of $(\lambda, \bar{\tau})$ space for which $\epsilon>\lambda^{-4} \eta_{\mathrm{L}}^{2}$ and $\xi>0$ are shown in Fig. 4.1 for both $\bar{\mu}>0$ and $\bar{\mu}<0$. The left-hand column of plots is for $\bar{\mu}>0$ while the right-hand column is for $\bar{\mu}<0$, in which case it is necessary to ensure that $\eta_{\mathrm{L}}=\sqrt{1+\bar{\alpha}-2 \bar{\beta}}-1 \geq 0$. In each case the region of $(\lambda, \bar{\tau})$ space in which a surface wave exists is marked with the + sign. In the left-hand column, Fig. 1 (a), (c), (e), the curves $\epsilon=0$ (continuous) and $\xi=0$ (dashed) are shown, and in Fig. 1 (b), (d), (f) the relevant curves are $\eta_{\mathrm{L}}=0$ (dashed) and $\xi=0$ (continuous). In the latter case $\eta_{\mathrm{L}}$ is positive only to the right of the curves $\eta_{\mathrm{L}}=0$. In (b) $\xi$ is positive between the two upper continuous curves and within the lower loop, while in (d) and (f) it is positive in between the three continuous curves.

We now provide a range of plots based on the solution of $\mathcal{N}=0$ from equation (4.79) in respect of the energy function (4.89) in dimensionless form to obtain $\zeta=\rho c^{2} / \mu$ as a function of $k h$. These are based on a representative, but by no means exhaustive, set of values of $\bar{\mu}, \bar{\mu}^{*}, \bar{\tau}, \bar{\tau}^{*}, \lambda, \lambda^{*}$ and the ratios $R=\rho^{*} \mu / \rho \mu^{*}$ and $r=\mu^{*} / \mu$ that illustrate the main features that can arise.

First, in Figs. 4.2 and 4.3, for the classical incompressible linearly elastic case with no initial stress, we show how $\zeta$ changes with $R$ and $r$. In Fig. 4.2 results for $R \leq 1$ are shown. In this case $\eta^{* 2}=1-R \zeta$ is positive since $\zeta=1$ is the upper limit for $\zeta$. In each of the subfigures in Figs. 4.2(a)(d) each of the curves passes through the classical limiting value $\zeta \approx$ 0.9126 when $k h=0$ (see [105] for detailed discussion and references to the incompressible classical theory) and there is only one propagation mode. Except for $r<1$ there is a cut-off value of $k h$ above which waves do not propagate, while for $r<1$ the wave speed is constant over a wide range of values of $k h$ and tends to the interfacial wave speed between two half-spaces as $k h \rightarrow \infty$.

In Figs. 4.2(e) there are two modes for $r=0.2$ and for only the first mode $\zeta \approx 0.9126$ when $k h=0$, and the second mode emerges at a positive value of $k h$, a mode that has a cut off value of $k h$ for low values of $k h$. For each of $r=1$ and $r=5$ there is only one mode. Finally, in Fig. 4.2(f), where $R=1$, there are two modes for each $r \neq 1$, but there is no dependence on $k h$ for $r=1$ because the half-space and layer materials are then identical, and the result is that for a half-space (non-dispersive). The results for 
$r=1, r<1$ and $r>1$ shown in Fig. 4.2 correspond to the continuous, thick continuous and dashed curves, respectively. No modes other than those shown appear at larger values of $k h$, and the general trend is the same for values of $r$ other than those for which results are shown here.

In Fig. 4.3 corresponding results are illustrated for $R=1$ and three values of $R>1$. In this case $\eta^{*}=0$ for $\zeta=1 / R$ and the dependence of $\zeta$ on $k h$ when $R>1$ separates into the regions $\zeta<1 / R\left(\eta^{* 2}>0\right)$ and $\zeta>1 / R$ $\left(\eta^{* 2}<0\right)$. In each case the lower branch passes through $\zeta \approx 0.9126$ at $k h=0$ for each value of $r$ but multiple other branches (modes) emerge at finite values of $k h$ except for $R=1$ in Fig. 4.3(a), which is the same as Fig. 4.2(f). Similar results are found for larger values of $R$.

Figure 4.4 serves to confirm that for the considered range of values used the effect of an initial stress is very similar to the effect of an initial stretch. Indeed, if the initial stress was calculated from a constitutive law for the same stretch then the effect would be identical. In each panel curves for three values of $r$, with $R=1, \bar{\mu}=\bar{\mu}^{*}=0$ are shown to illustrate the comparative effect of the relative stiffnesses of the layer and half-space. In Fig. 4.4(a) the stretches in the layer and half-space are set at unity $\left(\lambda=\lambda^{*}=1\right)$, the initial stress $\bar{\tau}^{*}=0$ in the layer and that in the half-space negative $(\bar{\tau}=-0.2)$, while Fig. $4.4(\mathrm{c})$ has $\lambda^{*}=1, \bar{\tau}=\bar{\tau}^{*}=0$ and $\lambda=0.9$. Thus, the results show that the qualitative features of compressive initial stress and compressive stretch in the half-space are the same. Similarly, by comparing Figs. 4.4(b) and 4.4(d) the same applies when the compressive stretch and initial stress are in the layer instead. In particular, when there is a compressive stretch or initial stress in the half-space only one surface wave branch exists, but if there is a compressive stretch or initial stress in the layer multiple modes are possible.

Figure 4.5 shows examples of corresponding results for tensile stretches and initial stresses. If these are in the half-space then multiple modes exist while for the layer only one mode arises.

These comparisons are limited to separate consideration of the stretches and the initial stresses, but when both stretches and initial stresses are included independently then the results can be significantly different. First, we note that if the material constants $\bar{\mu}$ and $\bar{\mu}^{*}$ are positive then their roles in (4.93) and (4.94) can be captured by varying $\bar{\tau}^{2}$ and $\bar{\tau}^{* 2}$, respectively. 
Indeed, a range of plots for $\bar{\mu}=1$ and $\bar{\mu}^{*}=1$ does not reveal qualitative differences from those shown in Figs. 4.4 and 4.5, and we therefore focus on negative values of $\bar{\mu}$ and/or $\bar{\mu}^{*}$, which can have a significant influence. To illustrate the effect of a negative $\bar{\mu}^{*}$, Fig. 4.6 shows results for $\bar{\mu}^{*}=-1.5$ with $\bar{\mu}=0, \bar{\tau}=\bar{\tau}^{*}=1, \lambda=\lambda^{*}=1$ and $R=1$, with $r=0.1$ and $r=10$ in the left and right figures, respectively. In this case multiple modes appear, the first corresponding to the half-space value at $k h=0$, but they are different from the multiple modes seen in Figs. 4.3-4.5. Although, for each $r$, the secondary modes appear to meet, when seen on a larger scale they are clearly completely separate.

Figure 4.7 shows two further examples, which are quite different from those in Fig. 4.6. First, in Fig. 4.7(a) with $\lambda=1, \lambda^{*}=0.8, R=1$, $\bar{\mu}=-1.5, \bar{\tau}=-0.5, \bar{\mu}^{*}=0, \bar{\tau}^{*}=-0.5, \zeta$ is plotted against $k h$ for three values of $r: 0.2,1,2$. In this case there are multiple branches for each $r$, the first passing through the relevant half-space surface wave value at $k h=0$ and the subsequent ones emerging at a non-zero value of $k h$. The new feature exemplified here is that as $r$ increases (i.e. the layer becomes stiffer relative to the half-space) $\zeta$ becomes zero at two values of $k h$ between which surface waves do not exist. A zero value of $\zeta$ is associated with the appearance of a static incremental mode of deformation arising at a point when the underlying configuration of the half-space/layer combination becomes unstable, resulting in surface undulations. The second example is shown in Fig. 4.7(b) with $\lambda=1.2, \lambda^{*}=0.8, R=0.8$, $\bar{\mu}=-1, \bar{\tau}=1, \bar{\mu}^{*}=0, \bar{\tau}^{*}=-0.5$ and for $r=0.2,0.5,2$ and again $\zeta$ is plotted against $k h$.

This leads, finally, to separate consideration of some situations in which $\zeta=0$. Since the roles of $\lambda$ and $\lambda^{*}$ are in many cases similar to those of $\bar{\tau}$ and $\bar{\tau}^{*}$, in Fig. 4.8 we fix $\lambda=\lambda^{*}=1$ and $\bar{\mu}=\bar{\mu} *=0, R=1$ and plot $\bar{\tau}^{*}$ against $k h$ for a series of values of $r$ with a negative and a positive $\bar{\tau}$ in the two panels. The curves in the two cases are very similar, with relatively small numerical differences. As the value of $\bar{\tau}^{*}$ is reduced from zero the uniform configuration remains stable until, for a given value of $r$, the appropriate curve is met, at which point surface undulations can appear that depend on $k h$. This occurs first for the larger values of $r$, i.e. for the stiffest layers. 
On the other hand, in Fig. 4.9, instead of fixing $\lambda=\lambda^{*}=1$, we fix $\bar{\tau}=\bar{\tau}^{*}=0$ and plot $\lambda$ against $k h$ for four separate values of $\lambda^{*}$ and, in each panel, several values of $r$. For the stiffest layers the results are very sensitive to values of compressive stretch, as Figs. 4.9(b), 4.9(c) and 4.9(d) demonstrate. In particular, a closed loop emerges for a small range of values of $k h$ and as the compression advances this loop merges with the rest of the curve for $r=6$ and expands upwards as the compression increases (not shown). Thus, for any given layer thickness the structure becomes very unstable for a range of wave numbers, and to prevent instability the stretch $\lambda$ in half-space should therefore be sufficiently large.

Note that the value of $\lambda$ at $k h=0$ corresponds to the classical instability value for a compressed neo-Hookean half-space under plane strain $(\approx 0.544)$ due to Biot and detailed in his book [104]; see also [105] for further discussion.

In this section we have selected particular values of the various stretch, initial stress and material parameters in order to illustrate the different features that can arise when considering the propagation of surface waves and loss of stability of a layered half-space. Clearly, many other possible combinations of these parameters could be adopted, but those we have chosen for illustration provide a representative range of possible results. 
(a)

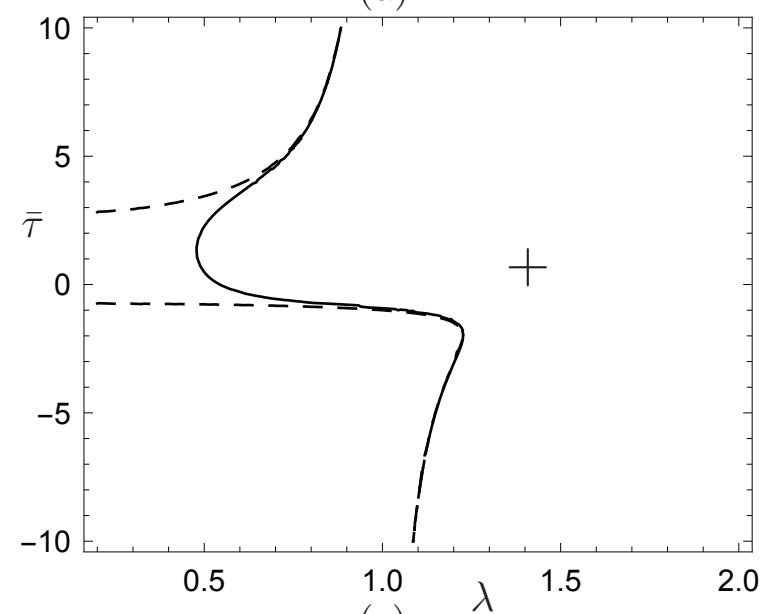

(c)

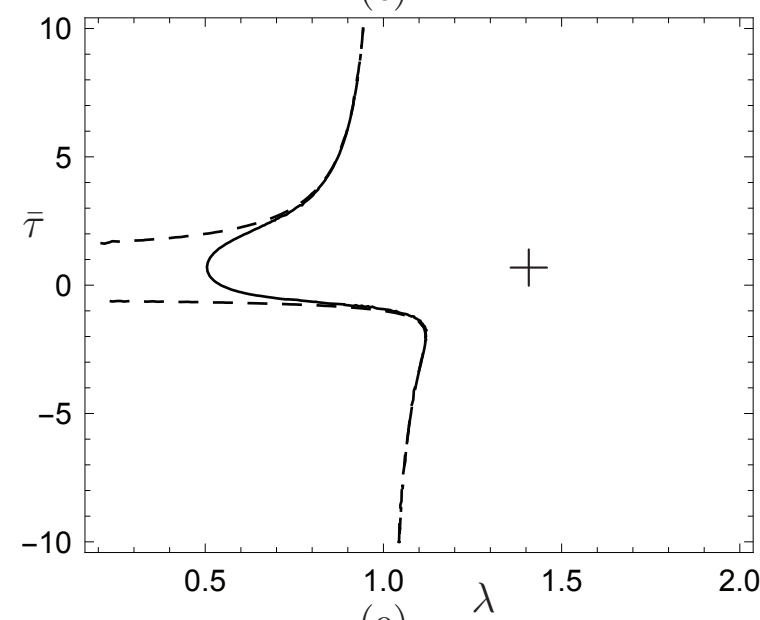

(e)

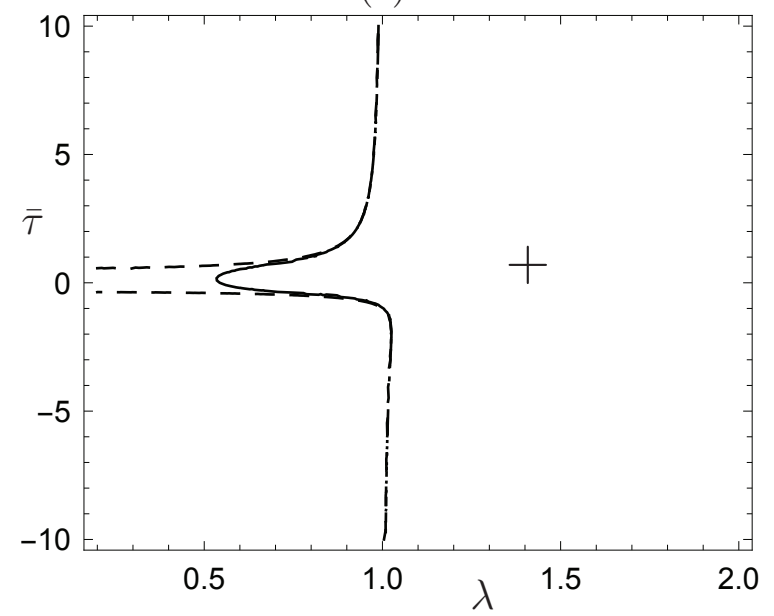

(b)

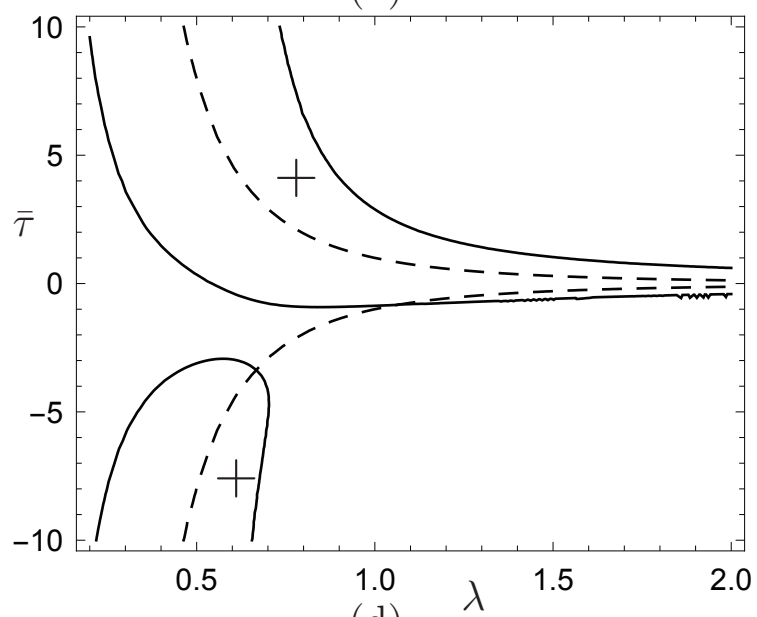

(d)

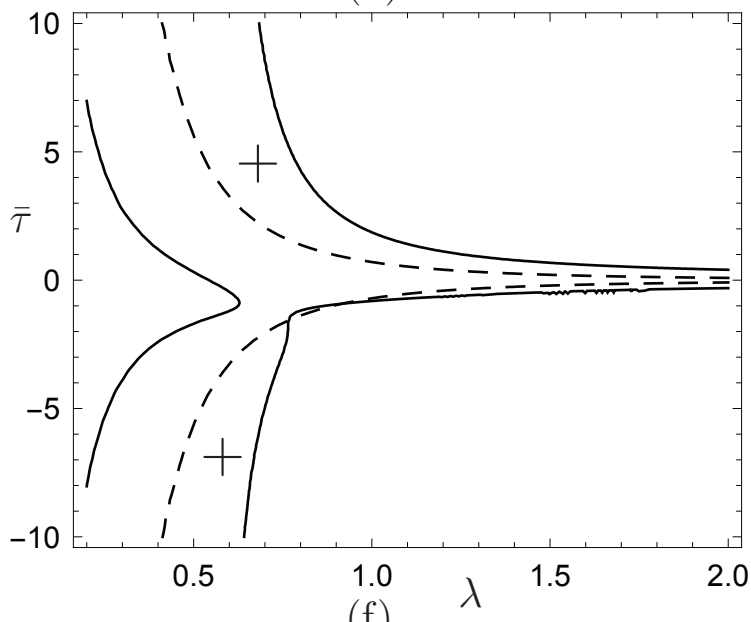

(f)

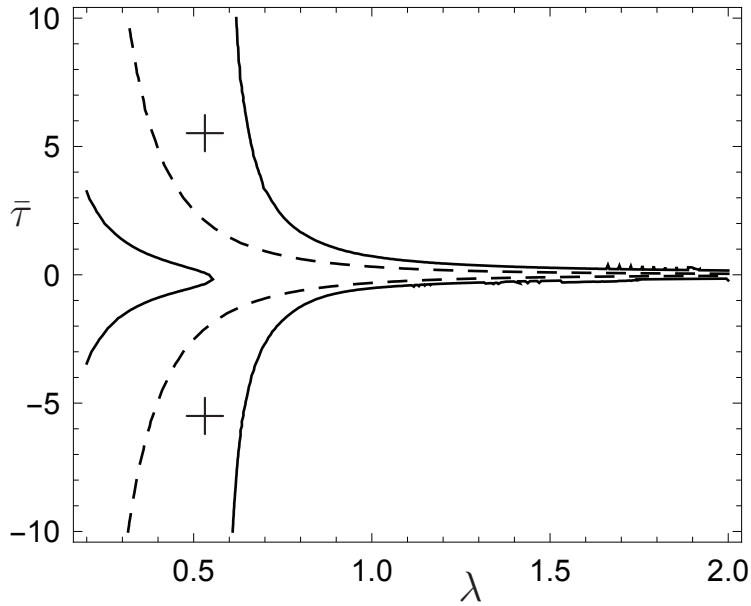

Figure 4.1: Plots of the curves $\epsilon=0$ (dashed curves) and $\xi=0$ (continuous curves) in $(\lambda, \bar{\tau})$ space for $\bar{\mu}=$ (a) 0.5 , (c) 1 , (e) 5 . The + sign indicates the regions of values of $\lambda$ and $\bar{\tau}$ for which surface waves exist and where $\xi>0$. Plots of the curves $\eta_{\mathrm{L}}=0$ (dashed) and $\xi=0$ (continuous) for $\bar{\mu}=(\mathrm{b})-0.5,(\mathrm{~d})-1$, (f) -5 . The + sign indicates the regions of values of $\lambda$ and $\bar{\tau}$ for which surface waves exist and where $\eta_{\mathrm{L}}>0$ and $\xi>0$. 
(a)

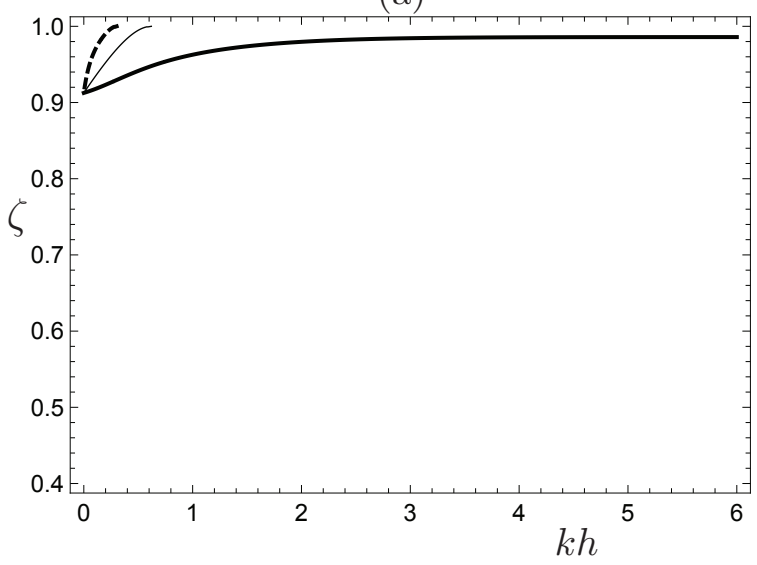

(c)

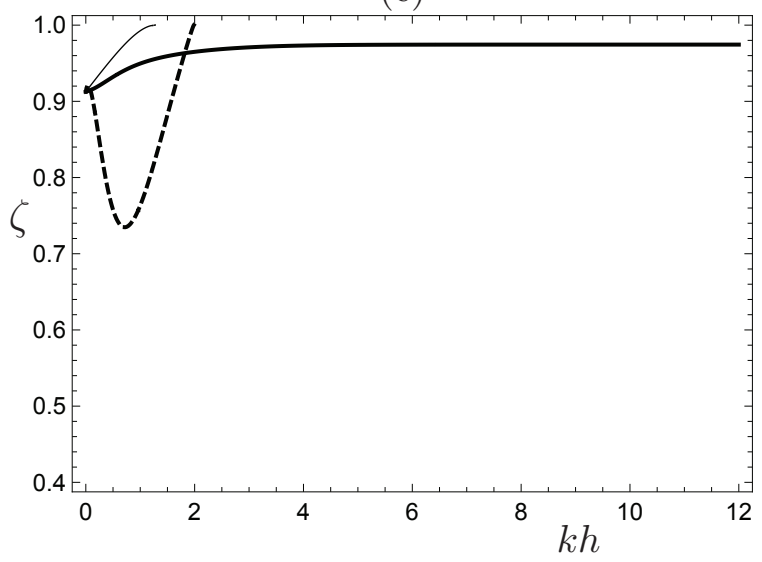

(e)

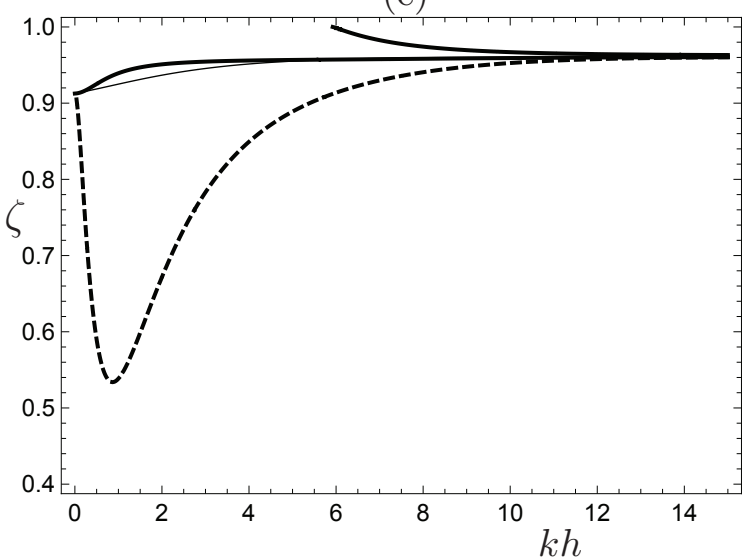

(b)

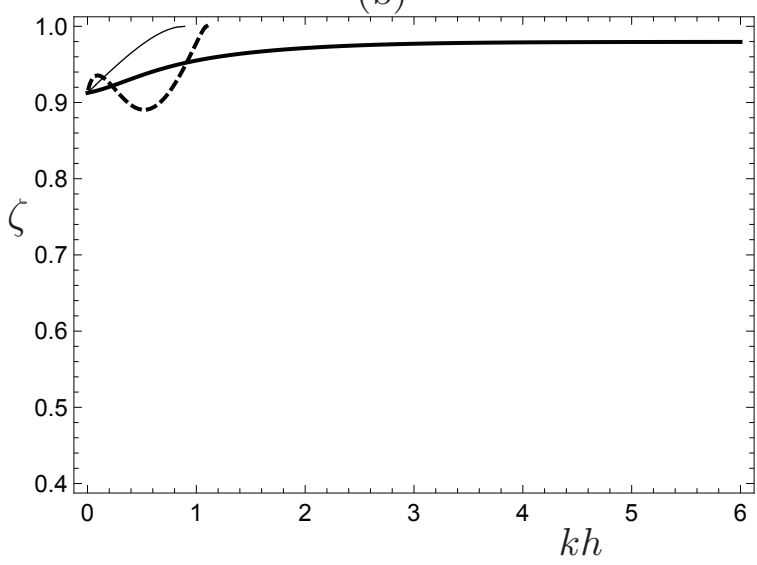

(d)

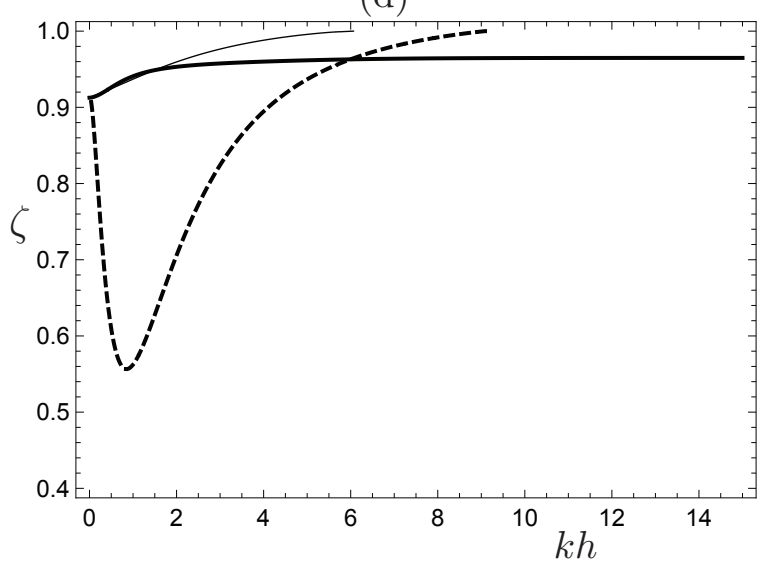

(f)

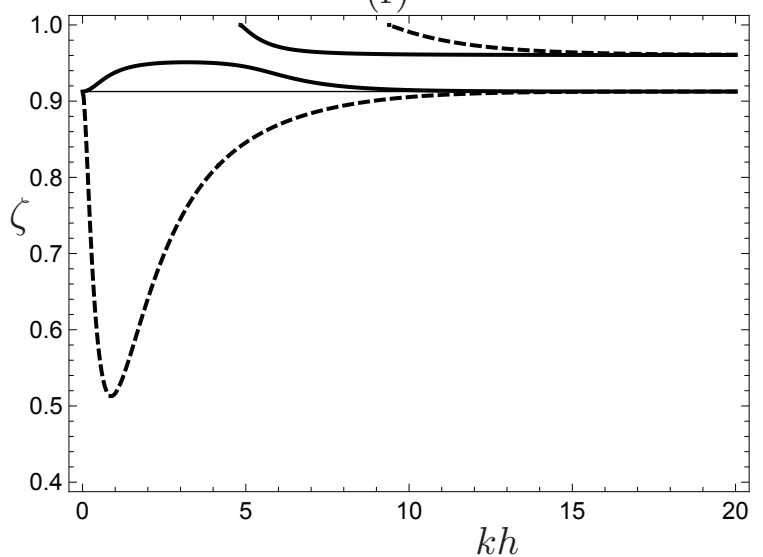

Figure 4.2: Plots of $\zeta=\rho c^{2} / \mu$ against $k h$ with $\lambda=\lambda^{*}=1, \bar{\tau}=\bar{\tau}^{*}=0$ and $r=0.2$ (thick continuous curves), $r=1$ (continuous curves), $r=5$ (dashed curves): (a) $R=0.1$; (b) $R=0.4$; (c) $R=0.6$; (d) $R=0.9$; (e) $R=0.95$; (f) $R=1$. 
(a)

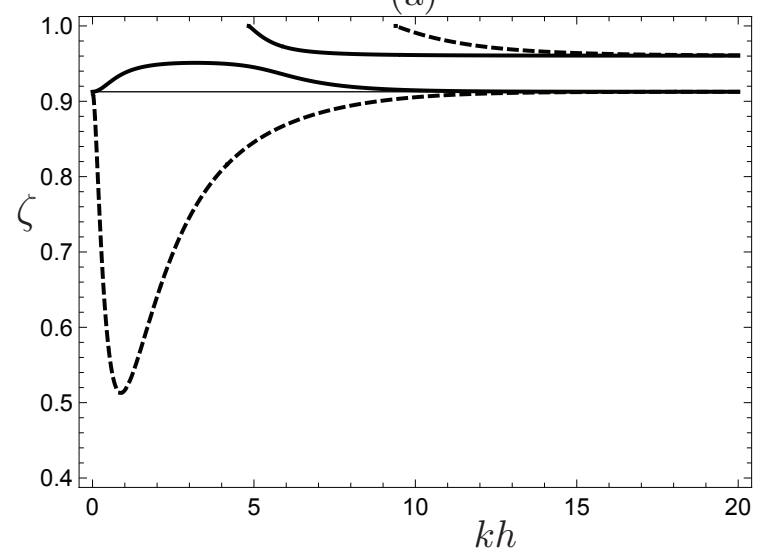

(c)

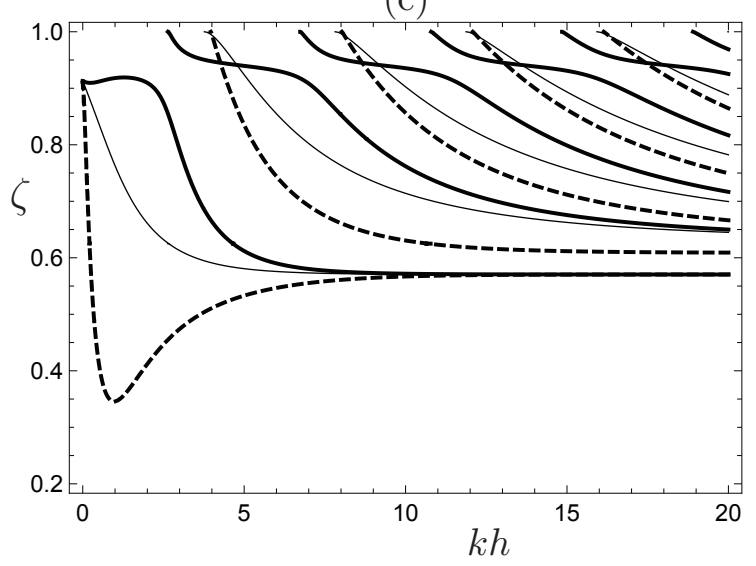

(b)

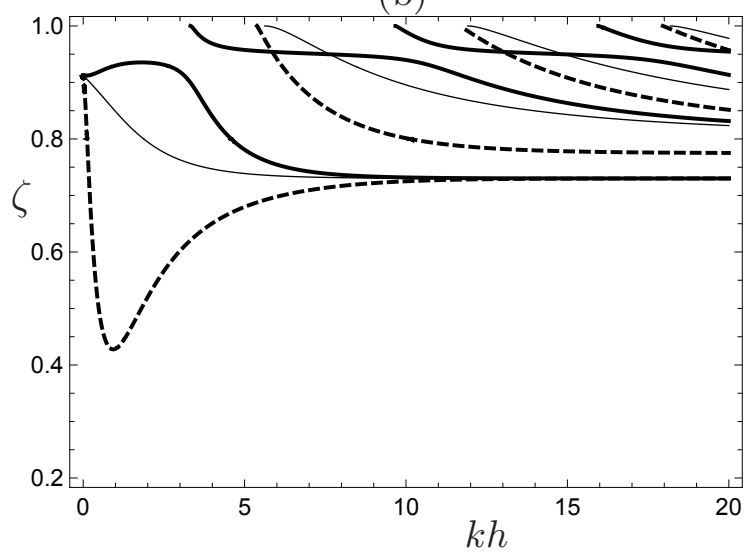

(d)

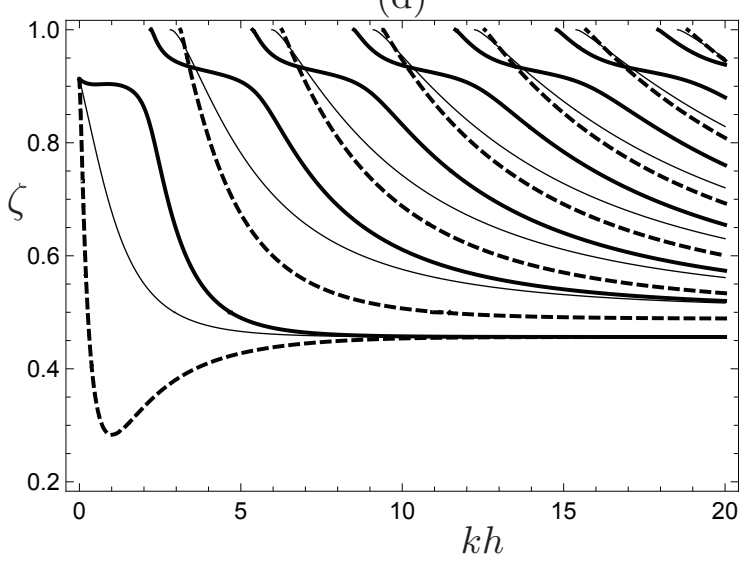

Figure 4.3: Plots of $\zeta=\rho c^{2} / \mu$ against $k h$ with $\lambda=\lambda^{*}=1, \bar{\tau}=\bar{\tau}^{*}=0$ and $r=0.2$ (thick continuous curves), $r=1$ (continuous curves), $r=5$ (dashed curves): (a) $R=1$; (b) $R=1.25$; (c) $R=1.6$; (d) $R=2$. 
(a)

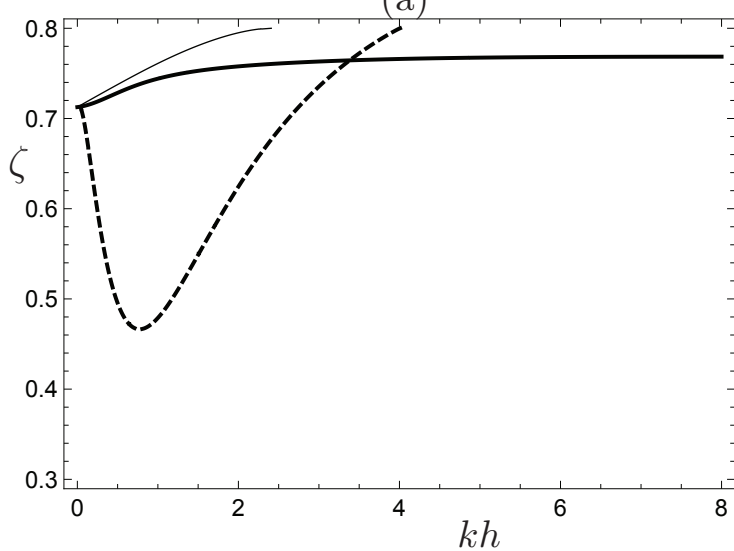

(c)

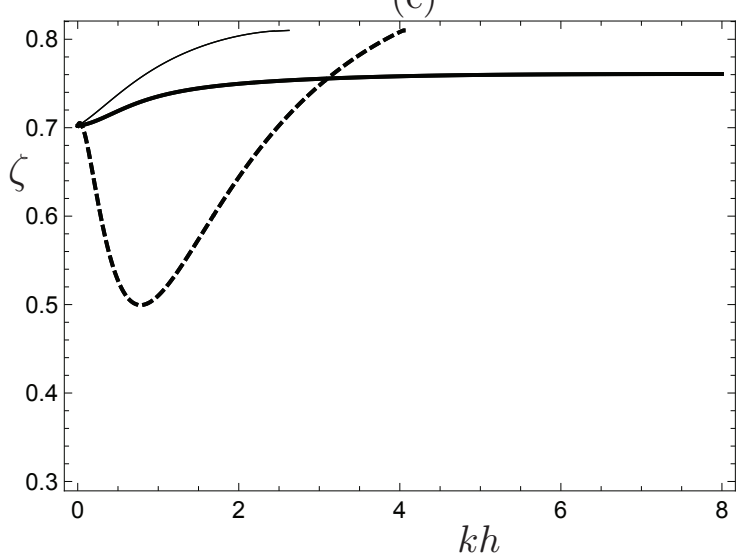

(b)

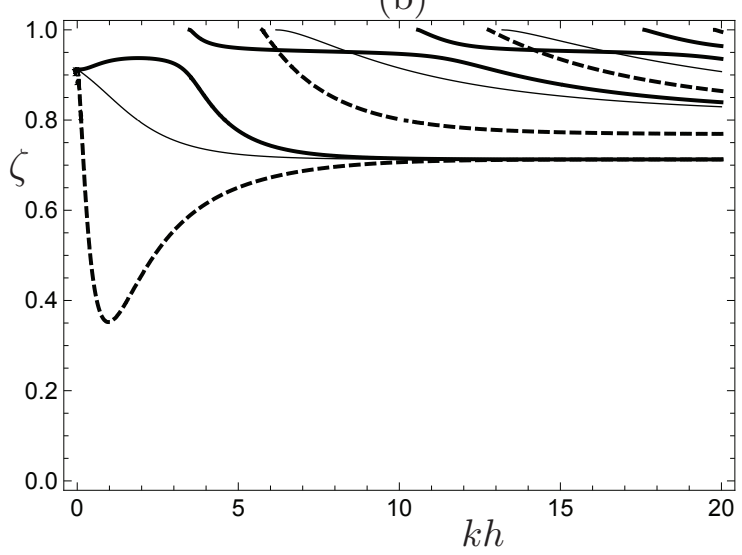

(d)

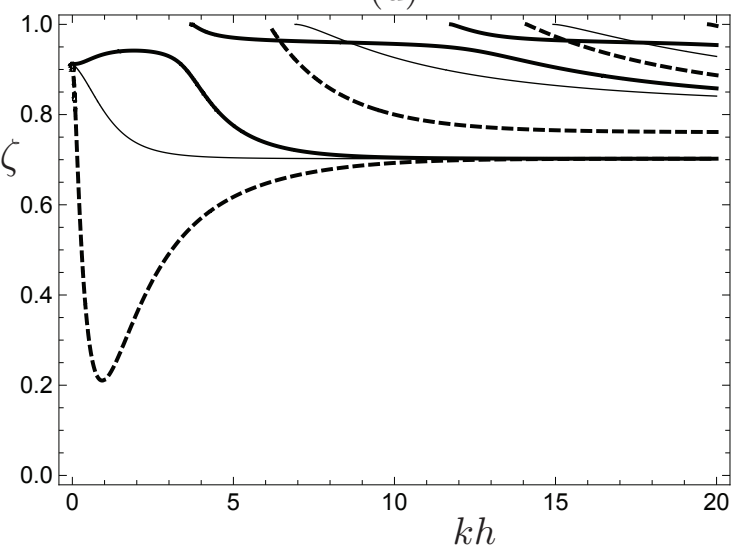

Figure 4.4: In each panel $\zeta=\rho c^{2} / \mu$ is plotted against $k h$ for $R=1$ and $r=0.2$ (thick continuous curves), $r=1$ (continuous curves), $r=5$ (dashed curves): (a) $\lambda=\lambda^{*}=1, \bar{\tau}=-0.2, \bar{\tau}^{*}=0$; (b) $\lambda=\lambda^{*}=1, \bar{\tau}=0, \bar{\tau}^{*}=-0.2$; (c) $\lambda=0.9, \lambda^{*}=1, \bar{\tau}=0, \bar{\tau}^{*}=0$; (d) $\lambda=1, \lambda^{*}=0.9, \bar{\tau}=0, \bar{\tau}^{*}=0$. 

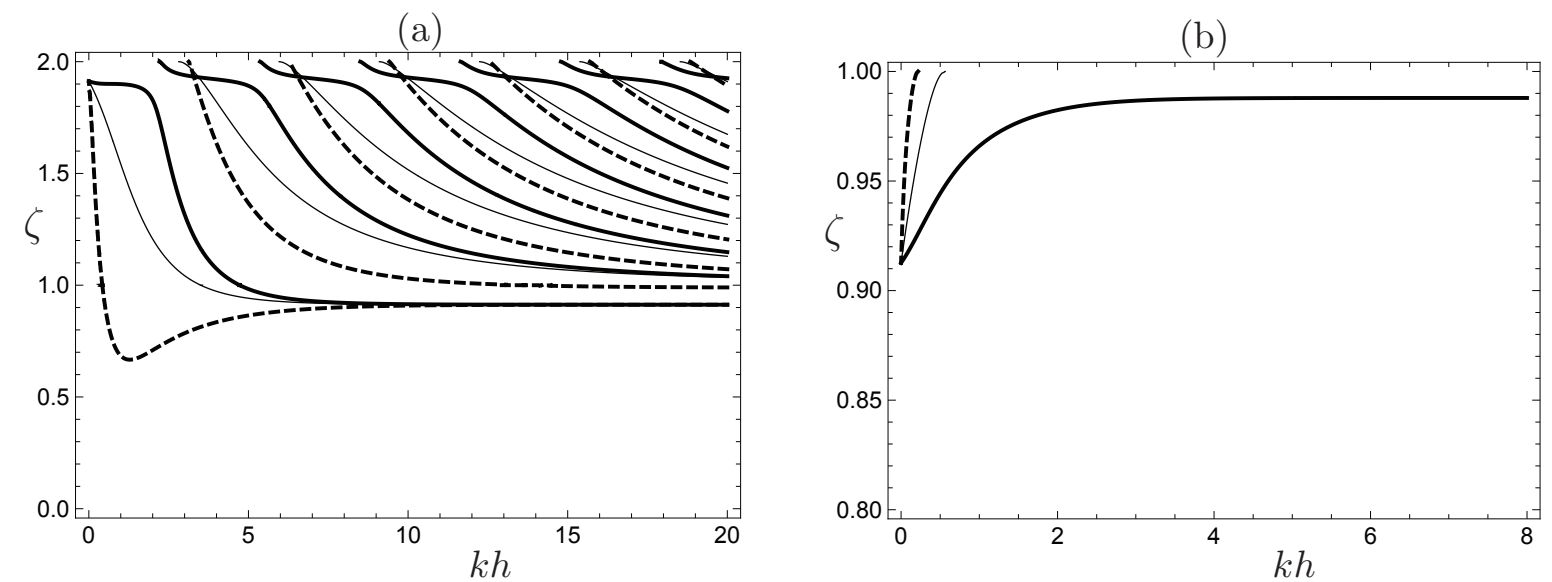

(c)

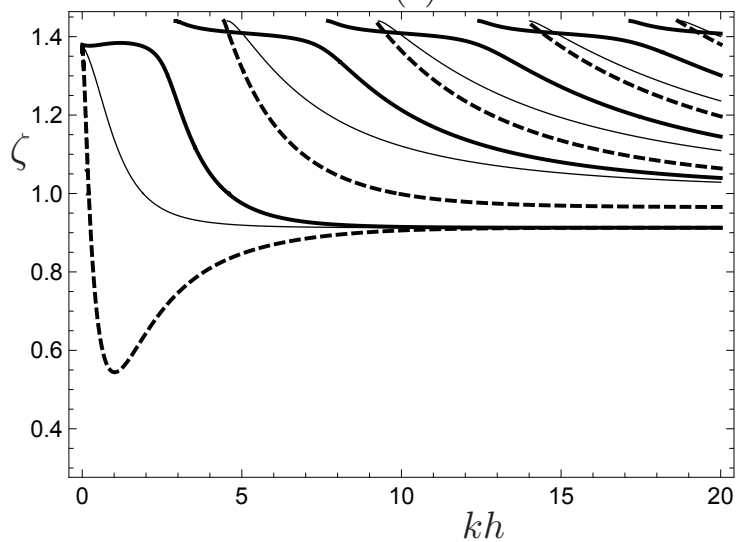

(d)

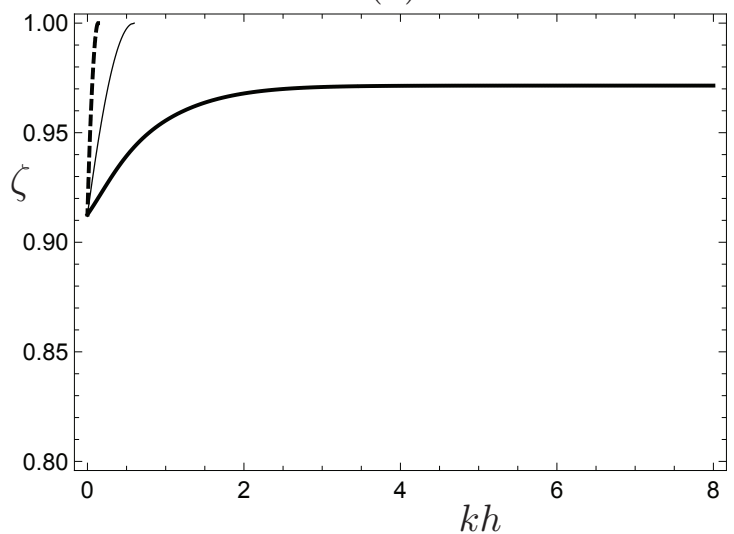

Figure 4.5: In each panel $\zeta=\rho c^{2} / \mu$ is plotted against $k h$ for $R=1$ and $r=0.2$ (thick continuous curves), $r=1$ (continuous curves), $r=5$ (dashed curves):(a) $\lambda=\lambda^{*}=1, \bar{\tau}=1, \bar{\tau}^{*}=0$; (b) $\lambda=\lambda^{*}=1, \bar{\tau}=0, \bar{\tau}^{*}=1$; (c) $\lambda=1.2, \lambda^{*}=1, \bar{\tau}=0, \bar{\tau}^{*}=0$; (d) $\lambda=1, \lambda^{*}=1.4, \bar{\tau}=0, \bar{\tau}^{*}=0$.

(a)

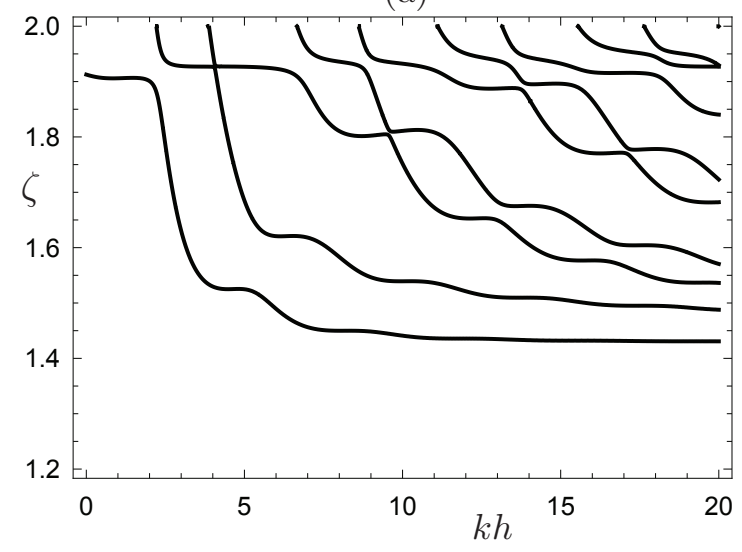

(b)

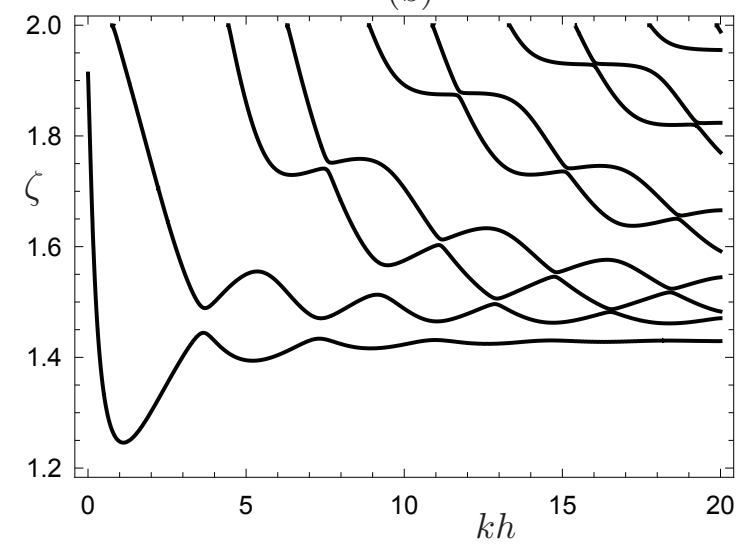

Figure 4.6: Plots of $\zeta$ against $k h$ for $\lambda=\lambda^{*}=1, R=1, \bar{\mu}=0, \bar{\tau}=1, \bar{\mu}^{*}=-1.5$, $\bar{\tau}^{*}=1$ : (a) $r=0.1$; (b) $r=10$. 
(a)

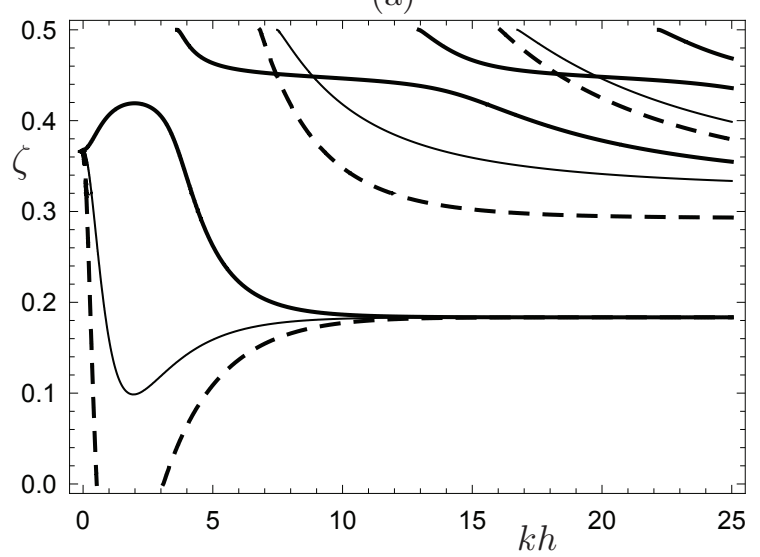

(b)

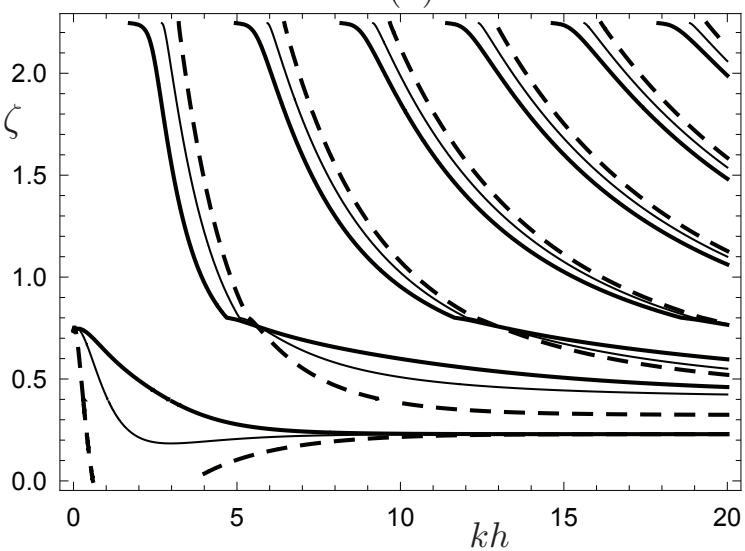

Figure 4.7: Plots of $\zeta$ against $k h$ : (a) $\lambda=1, \lambda^{*}=0.8, R=1, \bar{\mu}=-1.5$, $\bar{\tau}=-0.5, \bar{\mu}^{*}=0, \bar{\tau}^{*}=-0.5$, and $r=0.2,1,2$ corresponding to the thick continuous, continuous and dashed curves, respectively; (b) $\lambda=1.2, \lambda^{*}=0.8$, $R=0.8, \bar{\mu}=-1, \bar{\tau}=1, \bar{\mu}^{*}=0, \bar{\tau}^{*}=-0.5$, and $r=0.2,0.5,2$ corresponding to the thick continuous, continuous and dashed curves, respectively.

(a)

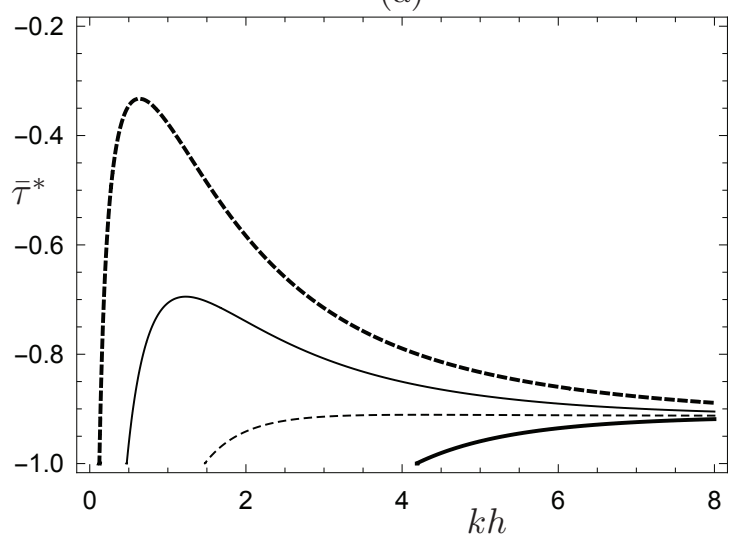

(b)

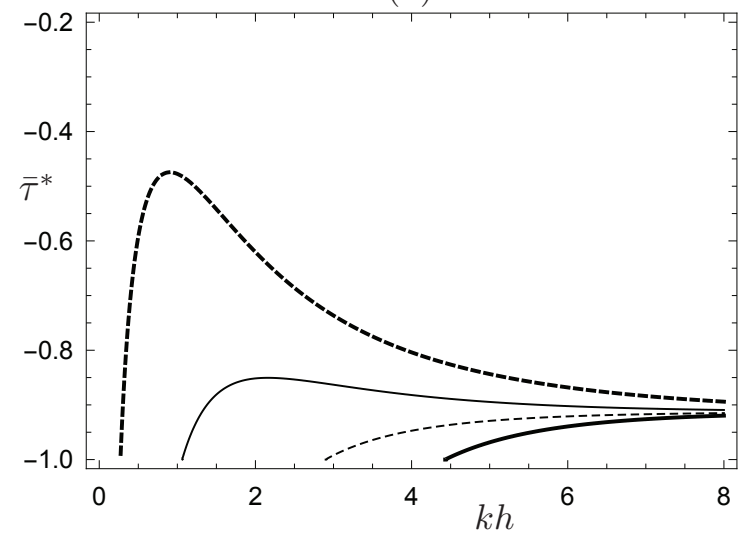

Figure 4.8: Plots of $\bar{\tau}^{*}$ against $k h$ for $\bar{\mu}=\bar{\mu}^{*}=0, \lambda=\lambda^{*}=1, \zeta=0$, $R=1$ : (a) $\bar{\tau}=-0.5$; (b) $\bar{\tau}=0.5$. In each of (a) and (b) curves are shown for $r=0.5,1.5,3,10$, respectively the thick continuous, dashed, continuous and thick dashed curves. 
(a)

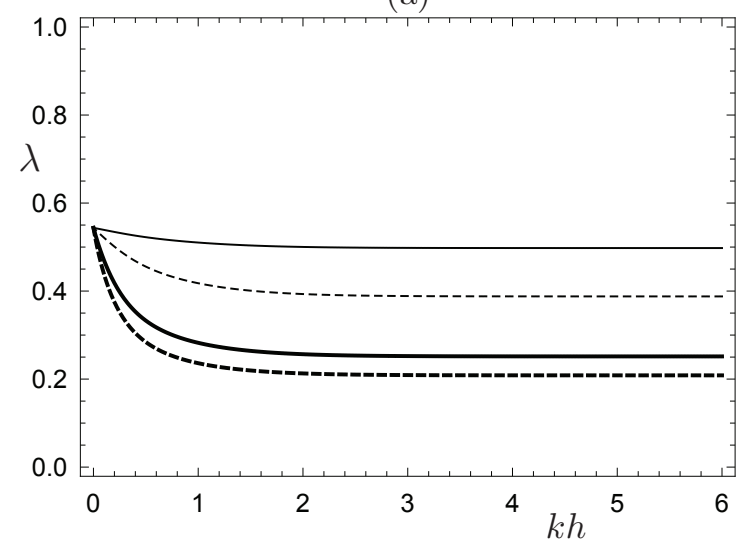

(c)

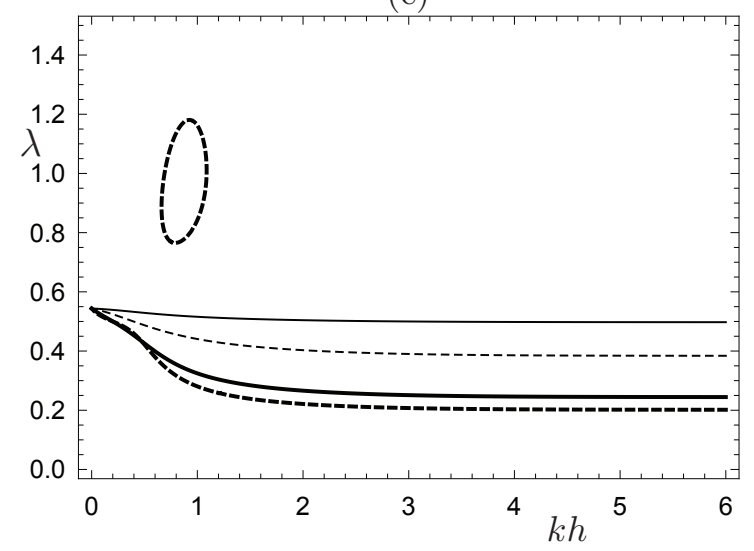

(b)

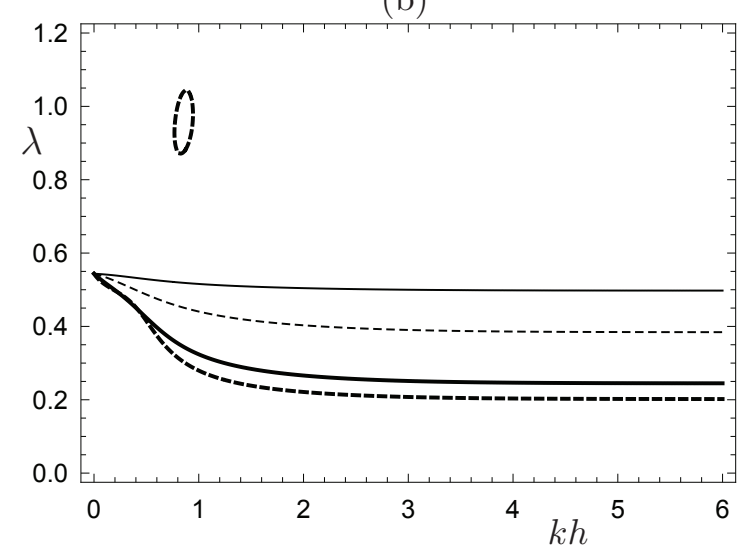

(d)

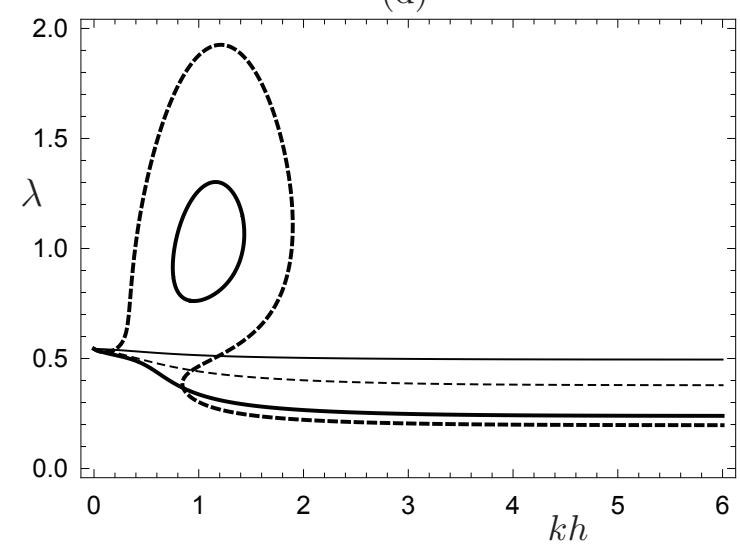

Figure 4.9: Plots of $\lambda$ vs $k h$ for $\bar{\mu}=\bar{\mu}^{*}=0, \bar{\tau}=\bar{\tau}^{*}=0, \zeta=0, R=1, r=$ $\mu^{*} / \mu=0.2,1,3.8,6$ in each plot. The panels (a), (b), (c), (d) correspond to $\lambda^{*}=1.4,0.854,0.85,0.8$, respectively. 
The following points briefly describe the most important contributions of this thesis as well as its conclusions.

- The propagation of non-principal Rayleigh waves in deformed transversely isotropic incompressible solids has been investigated in this thesis. The explicit secular equation for Rayleigh waves is obtained employing the method of polarization vector. This generalizes previous analyses dealing with isotropic incompressible pre-stressed materials. Exact formulas for the velocity of Rayleigh waves are derived for two special cases obeying that the wave propagation direction coincides with one of the principal direction of the pre-strain.

- The work in this thesis has been shown that the wave velocity depends strongly on the anisotropic character of the material model.

- The explicit and implicit secular equations for the speed of a (surface) Rayleigh wave propagating in a pre-stressed, doubly fiber-reinforced incompressible nonlinearly elastic half-space have been obtained in this thesis. The free surface coincides with one of the principal planes of the primary pure homogeneous strain, but the surface wave is not restricted to propagate in a principal direction. This generalizes previous results dealing with transversely isotropic nonlinearly elastic solids (see [62]). To 
illustrate the analysis, several strain energy functions have been considered. In particular, the materials under consideration are neo-Hookean models augmented with two functions, each one of them accounting for the existence of a unidirectional reinforcement. The functions endow the material with its anisotropic character and each one is referred to as a reinforcing model. In this thesis we consider two cases for the nature of the anisotropy: on the one hand, reinforcing models that have a particular influence on the shear response of the material $\left(I_{5}, I_{7}\right)$; on the other hand, reinforcing models that depend only on the stretch in the fiber direction $\left(I_{4}, I_{6}\right)$. The anisotropy influences the surface wave velocity of the isotropic base model.

- The work in this thesis shows that the influence on the wave velocity of the isotropic base model introduced by the invariants $I_{5}$ and $I_{7}$ is stronger than the one given by the invariants $I_{4}$ and $I_{6}$. models at hand are prototypes and have to be used with caution specially under fiber compression (see [92]).

- In this thesis we have also analyzed the combined effect of a uniform initial stress and finite deformation on the propagation of harmonic waves of infinitesimal amplitude in a half-space of elastic material with an overlying layer of uniform thickness and different material which is also subjected to a uniform initial stress and/or finite deformation. In particular, we have confirmed that when considered separately, not unexpectedly, the finite deformation and initial stress have similar consequences, but when they are both included independently the character of the waves is somewhat different.

- The work in this thesis also considers the special circumstances in which the wave speed vanishes, which corresponds to the emergence of small amplitude undulations of the layer and surface at critical values of the initial stress, deformation and material parameters. The analysis conducted here determines the point of bifurcation (or buckling) initiation, and we have not considered the post-bifurcation regime, which has also attracted recent attention but is more difficult 
to analyze in the general nonlinearly elastic context.

- However, several authors have examined post-buckling using a perturbation approach, mainly by considering the bonding of an unstretched stiff thin film to a stretched compliant substrate, which is then relaxed so that the film buckles. For example, [114] adopted a beam model for the film and a compressible neo-Hookean model for the substrate. Beyond the initiation of these so-called wrinkling deformations, in the post-bifurcation regime, the wrinkles can develop into different structures, including period doubling, folding, and crease and ridge formation, as exemplified in the work of [115], [116], [117] and references therein which involved combinations of theoretical analysis and finite element calculations supported by experimental observations of these characteristics.

- The results obtained in this thesis clearly show that finite deformation, initial stress and material properties have a strong influence on the mechanical characteristics of different types of structure, as exemplified by the layer/half-space substrate structure considered here. Detailed fully nonlinear analysis for other structures is therefore desirable in order to determine critical conditions corresponding to the onset of bifurcation and the post-bifurcation continuation into the fascinating patterns illustrated in the papers cited in the references. 


\section{Appendix. Torsion and inflation of a tube}

\section{A Kinematics and geometry}

Consider a material continuum which, when unstressed and unstrained, occupies the reference configuration $\mathcal{B}_{r}$. Let a typical material point in this configuration be identified by its position vector $\mathbf{X}$. The corresponding position vector in the deformed configuration $\mathcal{B}$ is denoted $\mathbf{x}$, and the deformation from $\mathcal{B}_{r}$ to $\mathcal{B}$ is written $\mathbf{x}=\chi(\mathbf{X})$, where the vector function $\chi$ is refered to as the deformation (attention is confined to aqua-static deformations here). The deformation gradient tensor, denoted $\mathbf{F}$, is given by

$$
\mathbf{F}=\operatorname{Grad} \chi(\mathbf{X})
$$

where Grad is the gradient operator with respect to $\mathbf{X}$. The associated right and left Cauchy-Green deformation tensors, denoted $\mathbf{C}$ and $\mathbf{B}$, respectively, are defined by

$$
\mathbf{C}=\mathbf{F}^{T} \mathbf{F}=\mathbf{U}^{2}, \quad \mathbf{B}=\mathbf{F F}^{T}=\mathbf{V}^{2}
$$

where ${ }^{T}$ signifies the transpose of a second-order tensor, $\mathbf{U}$ and $\mathbf{V}$, respectively, are the right and left stretch tensors, which are positive definite and symmetric and come from the polar decomposition $\mathbf{F}=\mathbf{R U}=\mathbf{V R}, \mathbf{R}$ being a proper orthogonal tensor. For a homogeneous incompressible nonlinearly isotropic elastic solid, the elastic stored energy (defined per unit volume) depends on only two invariants, which are the principal invariants of $\mathbf{C}$ (equivalently of $\mathbf{B}$ ), defined by

$I_{1}=\operatorname{tr}(\mathbf{C})=\lambda_{1}^{2}+\lambda_{2}^{2}+\lambda_{3}^{2}, \quad I_{2}=\frac{1}{2}\left[(\operatorname{tr} \mathbf{C})^{2}-\operatorname{tr}\left(\mathbf{C}^{\mathbf{2}}\right)\right]=\lambda_{1}^{2} \lambda_{2}^{2}+\lambda_{2}^{2} \lambda_{3}^{2}+\lambda_{3}^{2} \lambda_{1}^{2}$

where $\lambda_{i}>0, i \in\{1,2,3\}$ are the principal stretches, i.e. the eigenvalues of $\mathbf{U}$ and $\mathbf{V}$. The incompressibility constraint may be written as

$$
\operatorname{det} \mathbf{F}=1, \quad \text { or } \quad \lambda_{1} \lambda_{2} \lambda_{3}=1 \text {, }
$$

equivqlently in terms of $\mathbf{F}$ and the principal stretches, respectively. 


\section{A.1 Combined extension, inflation and torsion}

We now consider a circular cylindrical tube, which, in terms of cylindrical polar coordinates $(R, \Theta, Z)$, is defined by

$$
A \leq R \leq B, \quad 0 \leq \Theta \leq 2 \pi, \quad 0 \leq Z \leq L
$$

in the reference configuration $\mathcal{B}_{r}$, where $A$ and $B$ are the internal and external radii and $L$ in the length of the tube. The position vector $\mathbf{X}$ of a point of tube is given by

$$
\mathbf{X}=R \mathbf{E}_{R}+Z \mathbf{E}_{Z}
$$

where $\mathbf{E}_{R}$ and $\mathbf{E}_{Z}$ are the unit basis vectors associated with $R$ and $Z$, respectively. We also denote by $\mathbf{E}_{\Theta}$ the corresponding unit vector associated with $\Theta$.

The position vector $\mathbf{x}$ in the deformed tube is written

$$
\mathbf{x}=r \mathbf{e}_{r}+z \mathbf{e}_{z},
$$

where we make use of cylindrical polar coordinates $(r, \theta, z)$ in $\mathcal{B}$, which are associated with unit basic vectors $\mathbf{e}_{r}, \mathbf{e}_{\theta}, \mathbf{e}_{z}$, respectively. The (isochoric) deformation consisting of axial extension, radial inflations and a superimposed torsion is defined by

$$
r=\sqrt{a^{2}+\lambda_{z}^{-1}\left(R^{2}-A^{2}\right)}, \quad \theta=\Theta+\psi \lambda_{z} Z, \quad z=\lambda_{z} Z,
$$

where $\lambda_{z}$ is the (uniform) axial stretch of cylinder, and $\psi$ is the torsional deformation per unit deformed length. Plane cross sections of the tube remain plane, and an initial radius at axial coordinate $Z$ turns through an angle $\psi z$ after axial extension. The deformation geometry of the tube defined by

$$
a \leq r \leq b, \quad 0 \leq \theta \leq 2 \pi, \quad 0 \leq z \leq l=\lambda_{z} L .
$$

For this deformation, the deformation gradient is calculated explicitly as

$$
\mathbf{F}=\lambda_{r} \mathbf{e}_{r} \otimes \mathbf{E}_{R}+\lambda_{\theta} \mathbf{e}_{\theta} \otimes \mathbf{E}_{\Theta}+\lambda_{z} \mathbf{e}_{z} \otimes \mathbf{E}_{Z}+\lambda_{z} \gamma \mathbf{e}_{\theta} \otimes \mathbf{E}_{Z},
$$

where we have defined $\gamma$ as $\gamma=\psi r$ and $\lambda_{r}, \lambda_{\theta}$ and $\lambda_{z}$ are the principal stretches in the radial, azimuthal and axial directions prior to an application of torsion. In particular, $\lambda_{\theta}=r / R$. Nevertheless, the incompressibility constraint (A.4) $)_{2}$ becomes

$$
\lambda_{r} \lambda_{\theta} \lambda_{z}=1 \text {. }
$$

which is dependent of $\gamma$. The deformation tensor (A.2) are calculated as

$$
\begin{aligned}
\mathbf{C} & =\lambda_{r}^{2} \mathbf{E}_{R} \otimes \mathbf{E}_{R}+\lambda_{\theta}^{2} \mathbf{E}_{\Theta} \otimes \mathbf{E}_{\Theta}+\lambda_{z}^{2}\left(1+\gamma^{2}\right) \mathbf{E}_{Z} \otimes \mathbf{E}_{Z} \\
& +\gamma \lambda_{z} \lambda_{\theta}\left(\mathbf{E}_{\Theta} \otimes \mathbf{E}_{Z}+\mathbf{E}_{Z} \otimes \mathbf{E}_{\Theta}\right), \\
\mathbf{B} & =\lambda_{r}^{2} \mathbf{e}_{r} \otimes \mathbf{e}_{r}+\left(\lambda_{\theta}^{2}+\gamma^{2} \lambda_{z}^{2}\right) \mathbf{e}_{\theta} \otimes \mathbf{e}_{\theta}+\lambda_{z}^{2} \mathbf{e}_{z} \otimes \mathbf{e}_{z}+\gamma \lambda_{z}^{2}\left(\mathbf{e}_{\theta} \otimes \mathbf{e}_{z}+\mathbf{e}_{z} \otimes \mathbf{e}_{\theta}\right) .
\end{aligned}
$$


by refering to the expression for $\mathbf{C}$ above, the definition (A.3) $)_{1},(\mathrm{~A} .4)_{2}$ and (A.11), it may be deduced that the principal stretches $\lambda_{1}, \lambda_{2}, \lambda_{3}$, with a suitable ordering, can be taken to satisfy

$$
\lambda_{1}=\lambda_{r}, \quad \lambda_{2} \lambda_{3}=\lambda_{\theta} \lambda_{z}, \quad \lambda_{2}^{2}+\lambda_{3}^{2}=\lambda_{\theta}^{2}+\lambda_{z}^{2} .
$$

\section{B Equilibrium and residual stress}

Throughout this chapter, we assume that there are no body forces present. The Cauchy stress $\boldsymbol{\sigma}$ and the nominal stress $\mathbf{S}$ then satisfy the equilibrium equations

$$
\operatorname{div} \boldsymbol{\sigma}=\mathbf{0}, \quad \operatorname{Div} \mathbf{S}=\mathbf{0},
$$

respectively, where div and Div are the divergence operators with respect to $\mathbf{x} \in \mathcal{B}$ and $\boldsymbol{X} \in \mathcal{B}_{r}$, respectively, and are connected by $\boldsymbol{\sigma}=\mathbf{F S}$. Appropriate traction boundary $\partial \mathcal{B}_{r}$ of $\mathcal{B}_{r}$, but we do not specify these at this point.

We now assume that the reference configuration $\mathcal{B}_{r}$ is residually stressed, with the residual stress tensor denoted by $\boldsymbol{\tau}$. In this configuration, $\mathbf{S}=$ $\sigma=\tau$,i.e. there is no distinction between different measures of stress since the deformation is measured from $\mathcal{B}_{r}$.

The source of $\boldsymbol{\tau}$ does not concern us here. It may associated with some prior material processing, plastic deformation or manufacturing process, for example, and it assumed to be known. It arises in the absence of body forces and surface tractions on the boundary $\partial \mathcal{B}_{r}$ of the material body $\mathcal{B}_{r}$. It is also assumed that it is not accompanied by intrinsic couple stresses, so that it is symmetric $\left(\boldsymbol{\tau}^{T}=\boldsymbol{\tau}\right)$ and therefore the rotational balance equations are satisfied in $\mathcal{B}_{r}$ as well as the equilibrium equation

$$
\operatorname{Div} \boldsymbol{\tau}=\mathbf{0} .
$$

Since there are no surface tractions, then, by definition, $\tau$ must also satisfy the boundary condition

$$
\boldsymbol{\tau} \mathbf{N}=\mathbf{0} \quad \text { on } \quad \partial \mathcal{B}_{\mathbf{r}}
$$

Note that $\tau$ is a residual stress and is distinguished from other types of initial stress, which may associated with surface tractions. We emphasize at this point that residual stresses are necessarily non-uniform and geometry dependent, and the elastic response of a residually stressed material body is therefore inhomogeneous.

For the considered circular cylindrical geometry, it is appropriate to assume that only components of residual stress are $\tau_{R R}, \tau_{\Theta \Theta}$ and $\tau_{Z Z}$,i.e. there is no residual shear stress, an assumption that is compatible with the boundary condition (B.3) that the residual stress must satisfy. However, the $\mathrm{Z}$ component of the equilibrium equation and corresponding 
boundary condition $\tau_{Z Z} \equiv 0$. The remaining components, $\tau_{R R}$ and $\tau_{\Theta \Theta}$, can then be taken to depend only on $R$, and the non-trivial component of the equilibrium equation (B.2) is the radial equation

$$
\frac{d \tau_{R R}}{d R}+\frac{1}{R}\left(\tau_{R R}-\tau_{\Theta \Theta}\right)=0,
$$

with accompanying boundary condition from (B.3)

$$
\tau_{R R}=0 \quad \text { on } \quad R=A, B .
$$

Note that if $\tau_{R R}$ is known, then $\tau_{\Theta \Theta}$ is determined by (B.4) as $d\left(R \tau_{R R}\right) / d R$.

\section{Constitutive laws}

For a homogeneous incompressible elastic solid, the strain energy is a function only of the deformation gradient $\mathbf{F}$, and we write the strain energy function as $W(\mathbf{F})$ per unit volume, although, by objectively, $W$ depends on $\mathbf{F}$ only through the right Cauchy-Green tensor $\mathbf{C}$ defined in (A.2). The Cauchy and nominal stress tensors $\boldsymbol{\sigma}$ and $\mathbf{S}$ are given by

$$
\boldsymbol{\sigma}=\mathbf{F} \frac{\partial W}{\partial F}(\mathbf{F}, \boldsymbol{\tau})-q \mathbf{I}, \quad \mathbf{S}=\frac{\partial W}{\partial \mathbf{F}}-q \mathbf{F}^{-1},
$$

where $q$ is a Lagrange multiplier associated with the incompressible constraint (A.4) $)_{1}$ and $\mathbf{I}$ is the identity tensor in $\mathcal{B}$.

When the material is residually stressed, then it is also inhomogeneous. We consider its response still to be elastic relative to $\mathcal{B}_{r}$, with dependence on $\mathbf{X}$ through $\boldsymbol{\tau}(\mathbf{X})$ and we count for this by including $\boldsymbol{\tau}$ in the argument of $W$. Thus, we write

$$
W=W(\mathbf{F}, \boldsymbol{\tau}) .
$$

Note that this is automatically objective since $\boldsymbol{\tau}$ is unaffected by rotation in the deformed configuration $\mathcal{B}$ and $W$ depends on $\mathbf{F}$ only via $\mathbf{C}=\mathbf{F}^{T} \mathbf{F}$. We also note that if the material has no intrinsic preferred directions in $\mathcal{B}_{r}$ other than those associated with $\boldsymbol{\tau}$ (its eigenvectors), then the elastic properties of the material relative to $\mathcal{B}_{r}$ are anisotroic, i.e. $\boldsymbol{\tau}$ has an effect on the constitutive law analogous to that of a structure tensor associated with preferred directions. We shall elaborate on this point shortly.

The presence of $\boldsymbol{\tau}$ does not affect the formula (C.1) $)_{1}$ for the Cauchy stresses except by the dependence of $W$ on $\tau$. It given by

$$
\boldsymbol{\sigma}=\mathbf{F} \frac{\partial W}{\partial F}(\mathbf{F}, \boldsymbol{\tau})-q \mathbf{I}
$$

When $\mathbf{F}=\mathbf{I}$ this reduces to

$$
\boldsymbol{\tau}=\frac{\partial W}{\partial F}(\mathbf{I}, \boldsymbol{\tau})-q^{(r)} \mathbf{I},
$$


where $q^{(r)}$ is value of $q$ in $\mathcal{B}_{r}$. Equation (C.4) impose restrictions on the combination of $W$ and $\boldsymbol{\tau}$, that will be made more explicit in the following subsection.

Any symetric second-order tensor can be expressed in the spectral form in terms of its eigenvalues and eigenvectors. In particular, in terms of its eigenvalues, $\tau_{1}, \tau_{2}, \tau_{3}$ and (unit) eigenvectors $\mathbf{M}_{\mathbf{1}}, \mathbf{M}_{\mathbf{2}}, \mathbf{M}_{\mathbf{3}}$, say, $\boldsymbol{\tau}$ can be written

$$
\boldsymbol{\tau}=\tau_{1} \mathbf{M}_{\mathbf{1}} \otimes \mathbf{M}_{\mathbf{1}}+\tau_{2} \mathbf{M}_{\mathbf{2}} \otimes \mathbf{M}_{\mathbf{2}}+\tau_{3} \mathbf{M}_{\mathbf{3}} \otimes \mathbf{M}_{\mathbf{3}}
$$

and when $\boldsymbol{\tau}$ is included in $W$, each $\mathbf{M}_{\mathbf{i}} \otimes \mathbf{M}_{\mathbf{i}},(i=1,2,3)$ has a role similar to a structure tensor associated with a single preferred direction, as for a fibre-reinforced material. However, the $\mathbf{M}_{\mathbf{i}} \otimes \mathbf{M}_{\mathbf{i}}$ are not independent since they satisfy the identity

$$
\mathbf{M}_{1} \otimes \mathbf{M}_{1}+\mathbf{M}_{2} \otimes \mathbf{M}_{2}+\mathbf{M}_{3} \otimes \mathbf{M}_{3}=\mathbf{I},
$$

where $\mathbf{I}$ is the identity tensor in $\mathcal{B}_{r}$. Thus, $\boldsymbol{\tau}$ generates invariants, which contribute to the independent variables in the functional dependence of $W$. In particular, by specializing $\boldsymbol{\tau}$ to a rank-one tensor, say $\mathbf{M} \otimes \mathbf{M}$, we recover the invariants associated with transverse isotropy. We now consider an invariant formulation for $W$ with the invariants generated by $\mathbf{C}$ and $\boldsymbol{\tau}$.

\section{C.1 Invariant formulation}

With $W$ written explicitly as $W(\mathbf{C}, \boldsymbol{\tau})$, it is clear that $W$ is automatically objective. In the absence of any instrinsic material symmetry, $W$ is an isotropic function of the combination of $\mathbf{C}$ and $\boldsymbol{\tau}$ and must therefore satisfy the symmetry condition

$$
W\left(\mathbf{Q}^{*} \mathbf{C Q}^{* \mathbf{T}}, \mathbf{Q}^{*} \tau \mathbf{Q}^{* \mathbf{T}}\right)=W(\mathbf{C}, \boldsymbol{\tau}),
$$

for all orthogonal $\mathrm{Q}^{*}$ in $\mathcal{B}_{r}$. For an incompressible material, this is equivalent to the dependence of $W$ on nice invariants of $\mathbf{C}, \boldsymbol{\tau}$ and their combinations. These are typically taken to be, for $\mathbf{C}$,

$$
I_{1}=\operatorname{tr} \mathbf{C}, \quad I_{2}=\frac{1}{2}\left[(\operatorname{tr} \mathbf{C})^{2}-\operatorname{tr} \mathbf{C}^{2}\right]
$$

as for an isotropic material (note that the third invariant $I_{3}=\operatorname{det} \mathbf{C}$ is equal to 1 for an incompressible material). Similar for $\boldsymbol{\tau}$ (except that there is no counterpart for $\boldsymbol{\tau}$ of the incompressibility constraint $\operatorname{det} \mathbf{C}=1$ ),

$$
I_{4}=\left\{I_{41}, I_{42}, I_{43}\right\} \equiv\left\{\operatorname{tr} \boldsymbol{\tau}, \frac{1}{2}\left[(\operatorname{tr} \boldsymbol{\tau})^{2}-\operatorname{tr}\left(\boldsymbol{\tau}^{2}\right), \operatorname{det} \boldsymbol{\tau}\right]\right\},
$$

which are collectively denoted $I_{4}$. Then, we take the set of independent invariants involving the combination of $\mathbf{C}$ and $\boldsymbol{\tau}$ to be

$$
I_{5}=\operatorname{tr}(\boldsymbol{\tau} \mathbf{C}), \quad I_{6}=\operatorname{tr}\left(\boldsymbol{\tau} \mathbf{C}^{2}\right), \quad I_{7}=\operatorname{tr}\left(\boldsymbol{\tau}^{2} \mathbf{C}\right), \quad I_{8}=\operatorname{tr}\left(\boldsymbol{\tau}^{2} \mathbf{C}^{2}\right) .
$$


We emphasize that the above set of nine invariants or an equivalent set of alternative invariants, forms a complete set of invariants of $\mathbf{C}$ and $\boldsymbol{\tau}$ in three dimensions. When the dimension of the considered problem is reduced from three to two, such as for plane strain, the number of independent invariants is reduced.

We may now regard $W$ as a function of the above invariants. Thus, we take $W=W\left(I_{1}, I_{2}, I_{4}, I_{5}, I_{6}, I_{7}, I_{8}\right)$, and in the following we use the notation $W_{i}=\partial W / \partial I_{i}, i=1,2,4,5,6,7,8$. On evaluation of $\partial I_{i} / \partial \mathbf{F}$, $i=1,2,4,5,6,7,8$, the Cauchy stress tensor (C.3) then expands out as

$$
\begin{aligned}
\boldsymbol{\sigma} & =2 W_{1} \boldsymbol{B}+2 W_{2}\left(I_{1} \boldsymbol{B}-\boldsymbol{B}^{2}\right)+2 W_{5} \boldsymbol{\Sigma}+2 W_{6}(\boldsymbol{\Sigma} \boldsymbol{B}+\boldsymbol{B} \boldsymbol{\Sigma}) \\
& +2 W_{7} \boldsymbol{\Sigma} \boldsymbol{B}^{-1} \boldsymbol{\Sigma}+2 W_{8}\left(\boldsymbol{\Sigma} \boldsymbol{B}^{-1} \boldsymbol{\Sigma} \boldsymbol{B}+\boldsymbol{B} \boldsymbol{\Sigma} \boldsymbol{B}^{-1} \boldsymbol{\Sigma}\right)-q \mathbf{I}
\end{aligned}
$$

in which we have introduced the notation $\boldsymbol{\Sigma}=\mathbf{F} \tau \mathbf{F}^{T}$ for the Eulerian tensor which is push forward of $\tau$ from $\mathcal{B}_{r}$ to $\mathcal{B}$. We also recall that $\mathbf{B}=\mathbf{F F}^{T}$ is the left Cauchy-Green tensor.

The invariants of $\boldsymbol{\tau}$ are not affected by the deformation, while in the configuration $\mathcal{B}_{r}$, the other invariants reduce to

$$
I_{1}=I_{2}=3, \quad I_{5}=I_{6}=\operatorname{tr} \boldsymbol{\tau}, \quad I_{7}=I_{8}=\operatorname{tr}\left(\boldsymbol{\tau}^{2}\right) .
$$

By evaluating (C.11) in $\mathcal{B}_{r}$, we obtain the specialization of (C.4) in the form

$$
\boldsymbol{\tau}=\left(2 W_{1}+4 W_{2}-q^{(r)}\right) \mathbf{I}+2\left(W_{5}+2 W_{6}\right) \boldsymbol{\tau}+2\left(W_{7}+2 W_{8}\right) \boldsymbol{\tau}^{2},
$$

where all $W_{i i}, i \in\{1,2,4,5,6,7,8\}$, are evaluated for the invariants given by (C.12). Thus, we obtain the residual stress-dependent restrictions

$$
2 W_{1}+4 W_{2}-q^{(r)}=0, \quad 2\left(W_{5}+2 W_{6}\right)=1, \quad W_{7}+2 W_{8}=0,
$$

on the strain-energy function in $\mathcal{B}_{r}$ where again the $W_{i}$ are evaluated in $\mathcal{B}_{r}$.

\section{Application to combined extension, inflation, torsion}

For the considered deformation, with $\mathbf{C}$ given by $(\mathrm{A} .12)_{1}$ and residual stress components $\tau_{R R}$ and $\tau_{\Theta \Theta}$, the invariants are given by

$$
\begin{aligned}
I_{1} & =\lambda_{r}^{2}+\lambda_{\theta}^{2}+\lambda_{z}^{2}\left(1+\gamma^{2}\right), \quad I_{2}=\lambda_{\theta}^{2} \lambda_{z}^{2}+\lambda_{r}^{2} \lambda_{z}^{2}\left(1+\gamma^{2}\right)+\lambda_{r}^{2} \lambda_{\theta}^{2}, \\
I_{41} & =\tau_{R R}+\tau_{\Theta \Theta}, \quad I_{42}=\tau_{R R} \tau_{\Theta \Theta}, \quad I_{43}=0 \\
I_{5} & =\lambda_{r}^{2} \tau_{R R}+\lambda_{\theta}^{2} \tau_{\Theta \Theta}, \quad I_{6}=\lambda_{r}^{4} \tau_{R R}+\lambda_{\theta}^{2}\left(\lambda_{\theta}^{2}+\gamma^{2} \lambda_{z}^{2}\right) \tau_{\Theta \Theta}, \\
I_{7} & =\lambda_{r}^{2} \tau_{R R}^{2}+\lambda_{\theta}^{2} \tau_{\Theta \Theta}^{2}, \quad I_{8}=\lambda_{r}^{4} \tau_{R R}^{2}+\lambda_{\theta}^{2}\left(\lambda^{2}+\gamma^{2} \lambda_{z}^{2}\right) \tau_{\Theta \Theta}^{2} .
\end{aligned}
$$


They depend on just three independent deformation variables, which we take as $\lambda_{\theta}, \lambda_{z}$, and $\gamma$ together with $\tau_{R R}$ and $\tau_{\Theta \Theta}, \lambda_{r}$ being given by the incompressibility condition (A.11) in terms of $\lambda_{\theta}$ and $\lambda_{z}$. We therefore write the strain energy as a function of these variables, specially $\hat{W}\left(\lambda_{\theta}, \lambda_{z}, \gamma, \tau_{R R}, \tau_{\Theta \Theta}\right)$, which is defined by

$$
\hat{W}\left(\lambda_{\theta}, \lambda_{z}, \tau_{R R}, \tau_{\Theta \Theta}\right)=W\left(I_{1}, I_{2}, I_{4}, I_{5}, I_{6}, I_{7}, I_{8}\right),
$$

with $I_{1}, I_{2}, I_{4}, I_{5}, I_{6}, I_{7}, I_{8}$ given by (D.1). Then a straightforward calculation using the appropriate specialization of the components of the Cauchy stress in (C.11), the expression for $\mathbf{B}$ in (A.12) and the component matrix of $\boldsymbol{\Sigma}=\mathbf{F} \boldsymbol{\tau} \mathbf{F}^{T}$, which has the diagonal form $\operatorname{diag}\left[\lambda_{r}^{2} \tau_{\Theta \Theta}, \lambda_{r}^{2} \tau_{R R}, 0\right]$, leads to the compact formulas

$$
\begin{array}{ll}
\sigma_{\theta \theta}-\sigma_{r r}=\lambda_{\theta} \frac{\partial \hat{W}}{\partial \lambda_{\theta}}+\gamma \frac{\partial \hat{W}}{\gamma}, & \frac{\partial \hat{W}}{\partial \gamma}, \\
\sigma_{z z}-\sigma_{r r}=\lambda_{z} \frac{\partial \hat{W}}{\partial \lambda_{z}}-\gamma \frac{\partial \hat{W}}{\partial \gamma}, \\
\sigma_{\theta \theta}-\sigma_{r r}=\lambda_{\theta} \frac{\partial \hat{W}}{\partial \lambda_{\theta}}+\gamma \frac{\partial \hat{W}}{\gamma}, & \frac{\partial \hat{W}}{\partial \gamma}, \\
\sigma_{z z}-\sigma_{r r} & =\lambda_{z} \frac{\partial \hat{W}}{\partial \lambda_{z}}-\gamma \frac{\partial \hat{W}}{\partial \gamma},
\end{array}
$$

with $\sigma_{r \theta}=0$ and $\sigma_{r z}=0$. These formulas are the same as those for a fibrereinforced material with a single family of fibres except that in that case $\sigma_{r \theta}$ and $\sigma_{r z}$ are in general nonzero, although the content is different since $\hat{W}$ is different - it involves residual stress instead of the transverse isotropy associated with a single preferred direction. By default, the formulas also apply in isotropic case, with a recovered by setting the residual stress to zero.

\section{D.1 Equilibrium and boundary load}

Since $\lambda_{z}$ is a constant and $\lambda_{\theta}$ and $\gamma$ depend only on $r$ (equivalently $R$ ) while $\sigma_{r \theta}=\sigma_{r z}=0$, the equilibrium equation (B.1) -1 reduces to one scalar equation, namely

$$
r \frac{d}{d r}\left(\sigma_{r r}\right)+\sigma_{r r}-\sigma_{\theta \theta}=0,
$$

which can be intergrated to be

$$
\sigma_{r r}-\sigma_{r r}(a)=\int_{a}^{r}\left(\sigma_{\theta \theta}-\sigma_{r r}\right) \frac{d r}{r}
$$

where $\sigma_{r r}(a)$ is the value of $\sigma_{r r}$ on the boundary $r=a$. 
We now consider the situation in which the inner surface $r=a$ is subject to a presure $P$ and no traction is applied on $r=b$. Then $\sigma_{r r}(a)=-P$ and $\sigma_{r r}(b)=0$, and with the help of (D.3) ${ }_{1}$, equation (D.5) becomes

$$
P=\int_{a}^{b} \lambda_{\theta} \frac{\partial \hat{W}}{\partial \lambda_{\theta}} \frac{d r}{r}
$$

\section{E Two simple models with residual stress}

We restric attention to strain energy functions that consist of a basic neo-Hookean isotropic energy function supplemented by a term linear in either $I_{5}$ or $I_{6}$, and hence linear in $\tau$. Thus, we consider the strain-energy functions defined by

$$
W=\frac{1}{2} \mu\left(I_{1}-3\right)+\frac{1}{2}\left(I_{5}-t r \tau\right)
$$

and

$$
W=\frac{1}{2} \mu\left(I_{1}-3\right)+\frac{1}{4}\left(I_{6}-t r \boldsymbol{\tau}\right)
$$

where $\mu(>0)$ is a constant, which corresponds to the shear modulus in the undeformed configuration of a neo-Hookean (isotropic) material. The second term is the strain energy associated with the residual stress and we have taken account of the restriction $(\mathrm{C} .14)_{2}$. The invariants $I_{1}, I_{5}, I_{6}$ are given by (D.1).

The expression (C.11) for the Cauchy stress simplifies to

$$
\boldsymbol{\sigma}=\mu \mathbf{B}+\Sigma-q \mathbf{I}, \quad \boldsymbol{\sigma}=\mu \mathbf{B}+\frac{\mathbf{1}}{\mathbf{2}}(\mathbf{\Sigma} \mathbf{B}+\mathbf{B} \boldsymbol{\Sigma})-\mathbf{q} \mathbf{I},
$$

respectively, for these two energy functions, so that the residual stress in accounted for in somewhat different ways.

In conjunction with these models, it suffices to adopt a specific form of the residual stress component $\tau_{R R}$ that satisfies the boundary conditions (B.5). we therefore choose the simple form

$$
\tau_{R R}=\alpha(R-A)(R-B),
$$

and hence, from (B.4), $\tau_{\Theta \Theta}$ is given by

$$
\tau_{\Theta \Theta}=\alpha\left[3 R^{2}-2(A+B) R+A B\right],
$$

where $\alpha$ is constant which defines the strength of the residual stress. Note that $\tau_{R R}<0(>0)$. 
We obtain for (E.1)

$$
\begin{aligned}
& \lambda_{\theta} \hat{W}_{\lambda_{\theta}}=\mu\left(\lambda_{\theta}^{2}-\lambda_{\theta}^{-2} \lambda_{z}^{-2}\right)+\lambda_{\theta}^{2}-\lambda_{\theta}^{-2} \lambda_{z}^{-2} \tau_{R R}, \\
& \lambda_{z} \hat{W}_{\lambda_{z}}=\mu\left[\lambda_{z}^{2}-\lambda_{\theta}^{-2} \lambda_{z}^{-2}\right]-\lambda_{\theta}^{-2} \lambda_{z}^{-2} \tau_{R R}
\end{aligned}
$$

and for (E.2)

$$
\begin{aligned}
& \lambda_{\theta} \hat{W}_{\lambda_{\theta}}=\mu\left(\lambda_{\theta}^{2}-\lambda_{\theta}^{-2} \lambda_{z}^{-2}\right)+\lambda_{\theta}^{4} \tau_{\Theta \Theta}-\lambda_{\theta}^{-4} \lambda_{z}^{-4} \tau_{R R} \\
& \lambda_{z} \hat{W}_{\lambda_{z}}=\mu\left(\lambda_{z}^{2}-\lambda_{\theta}^{-2} \lambda_{2}^{-2}\right)-\lambda_{\theta}^{-4} \lambda_{z}^{-4} \tau_{R R}
\end{aligned}
$$

\section{F Linear stability analysis}

The stability of the hollow cylinder subjected to finite torsion is investigated using the method of incremental deformations superposed on finite strains. First, we define a perturbation of the basic axisymmetric solutions and, second, we solve the associated incremental problem for calculating the critical values for the torsion rate at the onset of the torsional instability.

\section{F.1 Definition of the incremental deformation}

Let us define an infinitesimal perturbation $\delta \mathbf{x}$ of the basic solution $\mathbf{x}$ as follows:

$$
\overline{\mathbf{x}}=\mathbf{x}+\delta \mathbf{x}=\chi(\mathbf{X}) \chi^{(1)}(\mathbf{x})
$$

where $\overline{\mathbf{x}}$ is the perturbed position vector, and $\chi^{(1)}: \mathcal{B B}_{r}$ is the incremental mapping that take the spatial position vector $\mathbf{x}$ into the perturbed configuration. If the displacement vector $\delta \mathbf{x}$ satisfies the condition $|\delta \mathbf{x}|^{2} \ll|\delta \mathbf{x}|$, for all $\mathbf{x} \in \mathcal{B}$, then we can refer to $\chi^{(\mathbf{1})}$ as an incremental deformation. The incremental displacement vector $\delta \mathbf{x}$, can be express as

$$
\delta \mathbf{x}=\chi^{(1)}(r, \theta, z)=u(r, \theta, z) \mathbf{e}_{r}+v(r, \theta, z) \mathbf{e}_{\theta}+w(r, \theta, z) \mathbf{e}_{z}
$$

where $u, v, w$ are the incremental displacement fiends. Accordingly, the spatial gradient associated to the incremental deformation, $\Gamma=\operatorname{grad} \chi^{(1)}(\mathbf{x})=\frac{\partial \chi^{(1)}(\mathbf{x})}{\partial \mathbf{x}}$, reads

$$
\Gamma=\left[\begin{array}{ccc}
u_{, r} & \left(u_{, \theta}-v\right) / r & u_{, z} \\
v_{, r} & \left(v_{, \theta}+u\right) / r & v_{, z} \\
w_{, r} & w_{, \theta} / r & w_{, z}
\end{array}\right]
$$

in the $\mathbf{e}_{i} \otimes \mathbf{e}_{j}$ basis, $(i, j)$ spanning over $\{r, \theta, z\}$, where the comma denotes the partial differentiation with respect to the argument. 
In the following, we will use barred variables in order to identify the perturbed quantities. From (C.14), the perturbed deformation gradient $\overline{\mathbf{F}}$ reads

$$
\overline{\mathbf{F}}=\mathbf{F}+\delta \mathbf{F}=\mathbf{F}+\Gamma \mathbf{F}
$$

where $\delta \mathbf{F}$ is the increment of the basis deformation gradient $\mathbf{F}$. The perturbed omial stress is given by

$$
\overline{\mathbf{S}}=\mathbf{S}+\dot{\mathbf{S}},
$$

where $\mathbf{S}$ is the nominal stress, and $\dot{\mathbf{S}}$ is its increment, which can calculate by simple differentiation rules.

In particular, its push-forward of $\dot{\mathbf{S}}_{0}$ in the perturbed configuration reads

$$
\dot{\mathbf{S}_{\mathbf{0}}}=\mathbf{F} \dot{\mathbf{S}}=\mathcal{A}_{0}^{1} \Gamma+q \Gamma-\dot{q} \mathbf{I},
$$

where $\dot{q}$ is increment of the Lagrage multiplier $q$, and $\mathcal{A}_{0}^{1}$ is the so called tensor of instantaneous moduli, which is given by

$$
\mathcal{A}_{0 h k l j}^{1}=F_{h i} F_{l \beta} \frac{\partial^{2} W}{\partial F_{k i} \partial F_{j \beta}}=\mu \delta_{k j} B_{h l}+\delta_{k j} \Sigma_{h l},
$$

corresponds to (E.1), and

$$
\mathcal{A}_{0 h k l j}^{1}=F_{h i} F_{l \beta} \frac{\partial^{2} W}{\partial F_{k i} \partial F_{j \beta}}=\mu \delta_{k j} B_{h l}+\delta_{k j} F_{h i} \tau_{i p} \tau_{p \beta} F_{\beta l},
$$

corresponds to (E.2), where $\delta_{k j}$ is the Kronecker delta. The incremental equilibrium equations in the spatial form can be written as

$$
\begin{array}{r}
\operatorname{div} \dot{\mathbf{S}}_{0}=\mathbf{0} \quad \text { in } \mathbf{B}^{\prime}, \\
\dot{\mathbf{S}}_{0}^{T} \cdot \mathbf{n}=\mathbf{0} .
\end{array}
$$

The incremental incompressibility constraint gives

$$
\operatorname{tr} \Gamma=0 .
$$

The bulk equations of the incremental boundary value problem are given by a system of four partial differential equations (PDEs), i.e. the three of them are

$$
\begin{aligned}
& \frac{\partial\left(r \dot{S}_{0 r r}\right)}{\partial r}+\frac{\partial \dot{S}_{0 \theta r}}{\partial \theta}+r \frac{\partial \dot{S}_{0 z r}}{\partial z}-\dot{S}_{0 \theta \theta}=0 \\
& \frac{\partial\left(r \dot{S}_{0 r \theta}\right)}{\partial r}+\frac{\partial \dot{S}_{0 \theta \theta}}{\partial \theta}+r \frac{\partial \dot{S}_{0 z \theta}}{\partial z}+\dot{S}_{0 \theta r}=0 \\
& \frac{\partial\left(r \dot{S}_{0 r z}\right)}{\partial r}+\frac{\partial \dot{S}_{0 \theta z}}{\partial \theta}+r \frac{\partial \dot{S}_{0 z z}}{\partial z}=0
\end{aligned}
$$

and the incremental incompressibility condition in equation (F.10)

$$
u_{, r}+\frac{u+v_{, \theta}}{r}+w_{, z}=0
$$




\section{F.2 Stroh formulation}

The Stroh formulation allows to transform the system of four PDEs in equations (F.11) and (F.12) with boundary conditions (F.9) into a system of six ordinary differential equations (ODEs) with the given initial conditions. Assuming variable separation, we consider the following expression of the incremental displacements:

$$
\{u, v, w, \dot{q}\}=\{U, V, W, Q\} \exp (i(m \theta+k z))
$$

where $m$ is the circumferential mode and $k=2 n \pi / L$ is the wavenumber of the tube in the longitudinal direction, with $m$ and $n$ being integers, and $U, V, W, Q$ are four scalar functions of $r$. Such a helical perturbation deforms the hollow cylinder.

From the equtions (F.6) and (F.13)the incremental stress components must have the following form

$$
\left\{\dot{S}_{0 r r}, \dot{S}_{0 r \theta}, \dot{S}_{0 r z}\right\}=\left\{S_{0 r r}, S_{0 r \theta}, S_{0 r z}\right\} \exp (i(m \theta+k z))
$$

where $S_{0 r r}, S_{0 r \theta}, S_{0 r z}$ are three scalar functions of $r$.

Now, let us define the displacement-traction vector $\eta$, as follows

$$
\boldsymbol{\eta}(r)=\left[\begin{array}{c}
\mathbf{U}(r) \\
i r \mathbf{S}(r)
\end{array}\right] \text { with }\left\{\begin{array}{l}
\mathbf{U}(r)=[U(r), V(r), W(r)]^{T} \\
\mathbf{S}(r)=\left[S_{0 r r}(r), S_{0 r \theta}(r), S_{0 r z}(r)\right]^{T}
\end{array}\right.
$$

The incremental equations can be written in the form

$$
\frac{d \boldsymbol{\eta}(r)}{d r}=\frac{i}{r} \mathbf{G}(r) \boldsymbol{\eta}(r),
$$

where is Stroh matrix, having the following block form

$$
\mathbf{G}=\left[\begin{array}{ll}
\mathbf{G}_{1} & \mathbf{G}_{2} \\
\mathbf{G}_{3} & \mathbf{G}_{1}^{+}
\end{array}\right]
$$

where

$\mathbf{G}_{1}=\left[\begin{array}{ccc}i & -m & -k r \\ -m \sigma_{1} & i \sigma_{1} & 0 \\ -k r \sigma_{2} & 0 & 0\end{array}\right], \mathbf{G}_{2}=\left[\begin{array}{ccc}0 & 0 & 0 \\ 0 & -1 / \alpha_{1} & 0 \\ 0 & 0 & -1 / \alpha_{2}\end{array}\right], \mathbf{G}_{3}=\left[\begin{array}{ccc}\kappa_{11} & i \kappa_{12} & i \kappa_{13} \\ -i \kappa_{12} & \kappa_{22} & \kappa_{23} \\ -i \kappa_{13} & \kappa_{23} & \kappa_{33}\end{array}\right]$,

in which

$$
\begin{aligned}
& \kappa_{11}=m^{2}\left(\nu_{1}-\alpha_{1} \sigma_{1}^{2}\right)+k^{2} r^{2}\left(\nu_{2}-\alpha_{2} \sigma_{2}^{2}\right)+\left(\mathcal{A}_{0 r r r r}^{1}+\mathcal{A}_{0 \theta \theta \theta \theta}^{1}\right)+2 \alpha_{1} \sigma_{1}+k r m\left(\mathcal{A}_{0 \theta r z r}^{1}+\mathcal{A}_{0 z r \theta r}^{1}\right) \\
& \kappa_{12}=m\left\{\mathcal{A}_{0 r r r}^{1}+\mathcal{A}_{0 \theta \theta \theta \theta}^{1}+\nu_{1}-\alpha_{1}\left[\left(\sigma_{1}-1\right)^{2}-1\right]\right\}+k r\left(\mathcal{A}_{0 \theta \theta z \theta}^{1}+\mathcal{A}_{0 z r \theta r}^{1}\right) \\
& \kappa_{13}=k_{z} r\left(\mathcal{A}_{0 r r r r}^{1}+\alpha_{1} \sigma_{1}\right) \\
& \kappa_{22}=m^{2}\left[\left(\mathcal{A}_{0 r r r r}^{1}+\mathcal{A}_{0 \theta \theta \theta \theta}^{1}\right)+2 \alpha_{1} \sigma_{2}\right]+k r m\left(\mathcal{A}_{0 \theta \theta z \theta}^{1}+\mathcal{A}_{0 z \theta \theta \theta}^{1}\right)+k^{2} r^{2} \nu_{3}+\nu_{1}-\alpha_{1} \sigma_{1}^{2} \\
& \kappa_{23}=m k_{z} r\left(\mathcal{A}_{0 r r r r}^{1}+2 \alpha_{1} \sigma_{1}\right) \\
& \kappa_{33}=m^{2} \alpha_{3}+k r^{2}\left(\mathcal{A}_{0 r r r r}^{1}+\mathcal{A}_{0 z z z z}^{1}+2 \alpha_{3} \sigma_{3}\right)+\operatorname{krm}\left(\mathcal{A}_{0 \theta z z z}^{1}+\mathcal{A}_{0 z z \theta z}^{1}\right)
\end{aligned}
$$


and

$$
\begin{array}{lll}
\nu_{1}=\mathcal{A}_{0 \theta r \theta r}^{1}, & \alpha_{1}=\mathcal{A}_{0 r \theta r \theta}^{1}, & \sigma_{1}=q / \alpha_{1}, \\
\nu_{2}=\mathcal{A}_{0 z r z r}^{1}, & \alpha_{2}=\mathcal{A}_{0 r z r z}^{1}, & \sigma_{2}=q / \alpha_{2}, \\
\nu_{3}=\mathcal{A}_{0 z \theta z \theta}^{1}, & \alpha_{3}=\mathcal{A}_{0 \theta z \theta z}^{1}, & \sigma_{3}=q / \alpha_{3} .
\end{array}
$$

Next, we introduce $\mathcal{N}$ as fundamental matrix solution to (F.16)

$$
\mathcal{N}(r)=\left[\boldsymbol{\eta}_{1}\left|\boldsymbol{\eta}_{2}\right| \ldots \mid \boldsymbol{\eta}_{6}\right] .
$$

It clearly satisfies

$$
\frac{d}{d r} \mathcal{N}(r)=\frac{i}{r} \mathbf{G}(r) \mathcal{N}(r)
$$

Let $\mathbf{M}(r, a)$ be the matricant solution to (F.16), the matrix such that

$$
\boldsymbol{\eta}(r)=\mathbf{M}(r, a) \boldsymbol{\eta}(a), \quad \mathbf{M}(a, a)=\mathbf{I}_{(6)},
$$

where

$$
\mathbf{M}(r, a)=\mathcal{N}(r) \mathcal{N}^{-1}(a) .
$$

Suppose that $\mathbf{M}$ has the form

$$
\mathbf{M}(r, a)=\left[\begin{array}{ll}
\mathbf{M}_{1}(r, a) & \mathbf{M}_{2}(r, a) \\
\mathbf{M}_{3}(r, a) & \mathbf{M}_{4}(r, a)
\end{array}\right]
$$

There is no traction on $r=b$

$$
\left[\begin{array}{l}
\mathbf{U}(r) \\
\mathbf{S}(r)
\end{array}\right]=\left[\begin{array}{ll}
\mathbf{M}_{1}(r, b) & \mathbf{M}_{2}(r, b) \\
\mathbf{M}_{3}(r, b) & \mathbf{M}_{4}(r, b)
\end{array}\right]\left[\begin{array}{c}
\mathbf{U}(b) \\
\mathbf{0}
\end{array}\right] .
$$

We obtain from (F.26)

$$
\begin{gathered}
\mathbf{U}(r)=\mathbf{M}_{1}(r, b) \mathbf{U}(b), \quad \mathbf{S}(r)=\mathbf{M}_{3}(r, b) \mathbf{U}(b) . \\
\mathbf{S}(r)=\mathbf{M}_{3} \mathbf{M}_{1}^{-1}=i \mathbf{z}(r) \mathbf{U}(r), \quad \text { where } \quad \mathbf{z}(r)=-i \mathbf{M}_{3} \mathbf{M}_{1}^{-1}
\end{gathered}
$$

is the (Hermitian) $3 \times 3$ surface impedance matrix.

$$
M(b, b)=\left[\begin{array}{ll}
\mathbf{I} & 0 \\
\mathbf{0} & I
\end{array}\right]=\left[\begin{array}{ll}
\mathbf{M}_{1}(b, b) & \mathbf{M}_{2}(b, b) \\
\mathbf{M}_{3}(b, b) & \mathbf{M}_{4}(b, b)
\end{array}\right]
$$

Equation (F.29) gives us $\mathbf{M}_{3}(b, b)=0$ or

$$
\mathbf{z}(b)=0 .
$$

On $r=b$ we have condition

$$
\operatorname{det}\left[\mathbf{z}(a)-P\left(\begin{array}{ccc}
G_{11} & G_{12} & G_{13} \\
0 & 0 & 0 \\
0 & 0 & 0
\end{array}\right)\right]=0
$$


Bibliography 
[1] J. Merodio, T. J. Pence, Kink surfaces in a Directionally Reinforced Neo-Hookean Material under Plane Deformation: I. Mechanical Equilibrium, Journal of Elasticity, 62 (2011) 119-144.

[2] J. Merodio, T. J. Pence, Kink surfaces in a Directionally Reinforced Neo-Hookean Material under Plane Deformation: II. Kink band stability and Maximally Disipative bandbroadening, Journal of Elasticity, 62 (2011) 145-170.

[3] J. Merodio, R. W. Ogden. Tensile instabilities and ellipticity in fiberreinforced compressible nonlinearly elastic solids, Int. J. Engng Sci., 43 (2005) 697-706.

[4] J. Merodio, On constitutive equations for fiber-reinforced nonlinearly viscoelastic materials, Mechanics Research Communications, 33 (2006) 764-770.

[5] J. Merodio and R.W.Ogden, On the equivalence of strong ellipticity in the material and spatial settings of finite elasticity, ZAMP, 56 (2006) 1096-1101.

[6] J. Merodio and K.R. Rajagopal, Constitutive equations for anisotropic nonlinearly viscoelastic materials, Mathematics and Mechanics of Solids, 12 (2007) 131-147.

[7] J. Merodio ,G. Saccomandi and I. Sgura, The Rectilinear Shear of Fiber-Reinforced Incompressible non-linearly Elastic Solids, International Journal of Non Linear Mechanics, issue in memory of Rivlin, 42 (2007) 342-354. 
[8] F. Kassianides, R. W. Ogden, J. Merodio and T. J. Pence, Azimuthal shear of a transversely isotropic elastic solid, Mathematics and Mechanics of Solids, 13 (2008) 690-724.

[9] D. Haughton and J. Merodio, The elasticity of arterial tissue affected by Marfanssyndrome, Mechanics Research Communications, 36 (2009) 659-66.

[10] L. Dorfmann, J. Merodio and R. W. Ogden, Non-smooth solutions in the azimuthal shear of an anisotropic nonlinearly elastic material, Journal of Engineering Mathematics, 68 Number 1 (2010) 27-36.

[11] J. Merodio and D. Haughton, Bifurcation of thick-walled cylindrical shells and the mechanical response of arterial tissue affected by Marfan's syndrome, Mechanics Research Communications, 37 (2010) $1-6$.

[12] Roger Bustamante and Jose Merodio, On simple constitutive restrictions for transversely isotropic nonlinearly elastic materials and isotropic magneto-sensitive elastomers, Journal of Engineering Mathematics, 68 Number 1 (2010) 15-26.

[13] J. Rodriguez and J. Merodio, A new derivation of the bifurcation conditions of inflated cylindrical membranes of elastic material under axial loading. Application to aneurysm formation, Mechanics Research Communications, 38 (2011) 203-210.

[14] Roger Bustamante and Jose Merodio, Constitutive structure in coupled nonlinear electroelasticity: invariant description and constitutive restrictions, International Journal of Non Linear Mechanics, 46 (2011) 1315-1323.

[15] Roger Bustamante and Jose Merodio, On weak formulations and their second variation in nonlinear electroelasticity, Mechanics Research Communications, 46 (2012) 15-19.

[16] A. A. Alhayani, J. A. Giraldo, J. Rodríguez and J. Merodio, Computational modeling of bulging of inflated cylindrical shells applicable to aneurysm formation and propagation in arterial wall tissue, FINEL, 73 (2013) 20-29. 
[17] M. El Hamdaoui, J. Merodio and R. W. Ogden, Loss of ellipticity in the combined helical, axial and radial elastic deformations of a fibrereinforced circular cylindrical tube, International Journal of Solids and Structures, 63 (2015) 99-108.

[18] M. El Hamdaoui, J. Merodio , Azimuthal shear of doubly fiber reinforced cylindrical tubes, Journal of Engineering Mathematics, 95 (2015) 347-357.

[19] J. Merodio, R. W. Ogden, Extension, inflation and torsion of a residually stressed circular cylindrical tube, Continuum Mechanics and Thermodynamics, 28 (2016) 157-174.

[20] J. Merodio, R. W. Ogden, On Tensile Instabilities and Ellipticity Loss in Fiber-Reinforced Incompressible Non-Linearly Elastic Solids, Mech. Research Comm, 32 (2005) 290-299.

[21] J. Merodio, R. W. Ogden, A Note on Strong Ellipticity for Transversely Isotropic Linearly Elastic Solids, QJMAM, 56 (2003) 589-591

[22] J. Merodio and J.M. Goicolea, On thermodynamically consistent constitutive equations for fiber-reinforcednonlinearly viscoelastic solids with application to biomechanics, Mechanics Research Communications, 34 (2007) 561-571.

[23] Michel Destrade and Jose Merodio Compression Instabilities of Tissues with Localized Strain Softening, International Journal of Applied Mechanics,3 (2011) 69-83

[24] J. Merodio, R.W.Ogden and J. Rodríguez The influence of residual stress on finite deformation elastic response, International Journal of Non Linear Mechanics, 56 (2013) 43-49.

[25] A. A. Alhayani, J. Rodríguez and J. Merodio, Competition between radial expansion and axial propagation during bulging of inflated cylindrical shells applicable to evolution of aortic aneurysms, International Journal of Engineering Science, 85 (2014) 74-89. 
[26] M. El Hamdaoui, J. Merodio , A note on finite elastic deformations of fiber reinforced nonlinearly elastic tubes, Archives of Mechanics, 67 (2015) 95-109.

[27] Ariel Ramírez-Torres, Reinaldo Rodríguez-Ramos, José Merodio, Julián Bravo-Castillero, Raúl Guinovart-Díaz and Juan Carlos López Alfonso, Action of body forces in tumor growth, International Journal of Engineering Science, 89 (2015) 18-34.

[28] Ariel Ramírez-Torres, Reinaldo Rodríguez-Ramos, José Merodio, Julián Bravo-Castillero, Raúl Guinovart-Díaz and Juan Carlos López Alfonso, Mathematical modeling of anisotropic avascular tumor growth, MRC, 69 (2015) 8-14.

[29] D. Guinovart-Sanjuan, Reinaldo Rodríguez-Ramos, Raúl GuinovartDíaz, Julián Bravo-Castillero, F.J. Sabina, José Merodio, F. Lebon, S. Dumont, Effective properties of regular elastic shell composites, Composites Part B: Engineering, 87 (2016) 12-20.

[30] H. Demirkoparan, J. Merodio, Bulging Bifurcation of Inflated Circular Cylinders of Doubly Fiber-Reinforced Hyperelastic Material under Axial Loading and Swelling, Mathematics and Mechanics of Solids, in press, (2016)

[31] L. Rayleigh, On waves propagating along the plane surface of an elastic solid, Proc. R. Soc. Lond. A 17 (1885) 4-11.

[32] S. D. M. Adams, R. V. Craster and D. P. Williams, Rayleigh waves guided by topography, Proc. R. Soc. A, 463 (2007) 531-550.

[33] A.N. Stroh, Dislocations and cracks in anisotropic elasticity, Philos. Mag., 3 (1958) 625-646.

[34] A.N. Stroh, Steady state problems in anisotropic elasticity, J. Math. Phys., 41 (1962) 77-103.

[35] D. M. Barnett, J. Lothe, K. Nishioka and R. J. Asaro, Elastic surface waves in anisotropic crystals: a simplified method for calculating Rayleigh velocities using dislocation theory, J. Phys. F: Met. Phys., 3 (1973) 1083-1096. 
[36] D.M. Barnett, J. Lothe, Consideration of the existence of surface wave (Rayleigh wave) solutions in anisotropic elastic crystals, J. Phys. F Metal Phys., 4 (1974) 671-686.

[37] D.M. Barnett, J. Lothe, Free surface (Rayleigh) waves in anisotropic elastic half- spaces: the surface impedance method, Proc. $R$. Soc. Lond. A, 402 (1985) 135-152.

[38] P. Chadwick, G.D. Smith, Foundations of the theory of surface waves in anisotropic elastic materials, in: C.-S. Yih (Ed.), Advances in Applied Mechanics, Academic Press, New York, 17 (1977) 303-376.

[39] T.C.T. Ting, Anisotropic Elasticity: Theory and Applications, Oxford University Press, New York, (1996).

[40] Y.B. Fu, A. Mielke, A new identity for the surface impedance matrix and its application to the determination of surface-wave speeds, Proc. R. Soc. Lond. A 458 (2002) 2523-2543

[41] A. Mielke, Y.B. Fu, Uniqueness of the surface-wave speed: a proof that is independent of the Stroh formalism, Math. Mech. Solids 9 (2004) 5-15.

[42] P. Chadwick, N. J. Wilson, The behaviour of elastic surface waves polarized in a plane of material symmetry II. Monoclinic media, Proc. R. Soc. Lond. A, 438 (1992) 207-223.

[43] R. M. Taziev, Dispersion relation for acoustic waves in an anisotropic elastic half-space, Sov. Phys. Acoust., 35 (1989) 535-538.

[44] T. C. T. Ting, The polarization vector and secular equation for surface waves in an anisotropic elastic half-space, Int. J. Solids Struct., 41 (2004) 2065-2083.

[45] V. G., Mozhaev, Some new ideas in the theory of surface acoustic waves in anisotropic media, in D. F. Parker and A. H., England (eds.), IUTAM Symposium on Anisotropy, Inhomogeneity and Nonlinearity in Solid Mechanics, Kluwer Academic Pub, Dordrecht, The Netherlands (1995) 455-462. 
[46] M. Destrade, The explicit secular equation for surface acoustic waves in monoclinic elastic crystals, J. Acoust. Soc. Am., 109 (2001) 13981402.

[47] T.C.T. Ting, An explicit secular equation for surface waves in an elastic material of general anisotropy, Q. J. Mech. Appl. Math., 55 (2002) 297-311.

[48] T. C. T. Ting, Explicit secular equation for surface waves in an anisotropic elastic half-space from Rayleigh to today, Surface Waves in Anisotropic and Laminated Bodies and Defects Detection NATO Science Series II: Mathematics, Physics and Chemistry, 163 (2005) 95-116.

[49] P. C. Vinh, R. W. Ogden, On formulas for the Rayleigh wave speed, Wave Motion, 39 (2004) 191-197.

[50] P. G. Malischewsky, A note on Rayleigh-wave velocities as a function of the material parameters, Geofisica Internacional, 45 (2004) 507509.

[51] R. W. Ogden, P. C. Vinh, On Rayleigh waves in incompressible orthotropic elastic solids, J. Acoust. Soc. Am., 115 (2004) 530- 533.

[52] P. C. Vinh, R. W. Ogden, Formulas for the Rayleigh wave peed in orthotropic elastic solids, Ach. Mech. 56 (2004) 247-265.

[53] P. C. Vinh, R. W. Ogden, On the Rayleigh Wave Speed in Orthotropic Elastic Solids, Meccanica, 40 (2005) 147-161.

[54] P. C. Vinh, On formulas for the velocity of Rayleigh waves in prestrained incompressible elastic solids, Trans. ASME J. Appl. Mech., 77 (2) (2010) 021006, 9 pages.

[55] P. C. Vinh, On formulas for the Rayleigh wave velocity in pre-stressed compressible solids, Wave Motion 48 (2011) 614-625.

[56] A. P. Kiselev, G. A. Rogerson, Laterally dependent surface waves in an elastic medium with a general depth dependence, Wave Motion, 46 (8) (2009) 539-547. 
[57] A. P. Kiselev, D. F. Parker, Omni-directional Rayleigh, Stoneley and Scholte waves with general time dependence, Proc. R. Soc. Lond. A, 466 (2010) 2241-2258.

[58] D. A. Prikazchikov, Rayleigh waves of arbitrary profile in anisotropic media, Mech. Res. Comm., 50 (2013) 83-86.

[59] D. F. Parker, The Sroh formulism for elastic surfacewaves of general profile, Proc. R. Soc. Lond. A, 469 (2013) 20130301.

[60] Y. B. Fu, G. A. Rogerson, W. F. Wang, Surface waves guided by topography in an anisotropic elastic half-space, Proc. R. Soc. Lond. A, 489 (2003) 20120371,

[61] J. Kaplunov, E. Nolde, D. A. Prikazchikov, A revisit to the moving load problem using an asymptotic model for the Rayleigh wave, Wave Motion, 71 (2010) 440-451.

[62] P.C. Vinh, J. Merodio, T.T. Hue, N. Nguyen, Non-principal Rayleigh waves in deformed incompressible transversely isotropic elastic halfspaces, IMA J. Appl. Math., 79 (2014) 915-928.

[63] N. T. Nam, J. Merodio, P. C. Vinh, The secular equation for nonprincipal Rayleigh waves in deformed incompressible doubly fiberreinforced nonlinearly elastic solids, Int. J. Non-Lin. Mech., 84 (2016) 23-30.

[64] N.T. Nam, J. Merodio, R.W. Ogden, P.C. Vinh, The effect of initial stress on the propagation of surface waves in a layered half-space, Int. J. Solid. Struct., 88-89 (2016) 88-100.

[65] D. A. Prikazchikov, G. A. Rogerson, On surface wave propagation in incompressible, transversely isotropic, pre-stressed elastic half-spaces, Int. J. of Eng. Sci., 42 (2004) 967-986.

[66] F. G. Makhort, Some acoustic Rayleigh-wave relations for stress determination in deformed bodies, Prikl. Mekh., 14 (10) (1978) 123125 . 
[67] F. G. Makhort, O. I. Guscha and A. A. Chernoonchenko, Theory of acoustoelasticity of Rayleigh surface waves, Prikl. Mekh., 26 (4) (1990) 35-41.

[68] M. Hirao, H. Fukuoka, K. Hori, Acoustoelastic effect of Rayleigh surface wave in isotropic material, J. Appl. Mech., 48 (1981) 119-124.

[69] D. Husson, A perturbation theory for the acoustoelastic effect of surface waves, J. Appl. Phys., 57 (5) (1985) 1562-1568.

[70] P. P. Delsanto, A. V. Clark, Rayleigh wave propagation in deformed orthotropic materials, J. Acoust. Soc. Am., 81 (4) (1987) 952-960.

[71] M. Dyquennoy, M. Ouaftouh, M. Ourak, Determination of stresses in aluminium alloy using optical detection of Rayleigh waves, Ultrasonics, 37 (1999) 365-372.

[72] M. Dyquennoy, D. Devos and M. Ouaftouh, Ultrasonic evaluation of residual stresses in flat glass tempering: Comparing experimental investigation and numerical modeling, J. Acoust. Soc. Am., 119 (6) (2006) 3773-3781.

[73] E. Hu, Y. He and Y. Chen, Experimental study on the surface stress measurement with Rayleigh wave detection technique, Appl. Acoust., 70 (2009) 356-360.

[74] M. Destrade, M. D. Gilchrist, G. Saccomandi, Third- and fourth-order con-stants of incompressible soft solids and the acousto-elastic effect, J. Acoust. Soc. Am., 127 (5) (2010) 2759-2763

[75] P. C. Vinh and Jose Merodio, On acoustoelasticity and the elastic constants of the soft biological tissues, J. Mech. Mater. Struct., 8 (2013) 359-367.

[76] P. C. Vinh and Jose Merodio, Wave velocity formulas to evaluate elastic constants of soft biological tissues, J. Mech. Mater. Struct., 8 (2013) 51-64.

[77] G. A. Holzapfel, T. C. Gasser, R. W. Ogden, A new constitutive framework for arterial wall mechanics and a comparative study of material models, J. Elast. 61 (2000) 1-48. 
[78] M. Destrade, M. D. Gilchrist, R. Ogden, Third- and fourth-order elasticity of biological soft tissues, J. Acoust. Soc. Am., 127 (2010), 2103-2106.

[79] J. Merodio, R. W. Ogden, Mechanical response of fiber-reinforced incompressible nonlinearly elastic solids, Int. J. Nonlinear Mech., 40 (2005) 213-227.

[80] J. Merodio, P. Neff. A note on tensile instabilities and loss of ellipticity for a fiber-reinforced nonlinearly elastic solid under plane deformation, Archive of Mechanics, 58 (2006) 293-303.

[81] J. Merodio, G. Saccomandi, Remarks on cavity formation in fiberreinforced incompressible non-linearly elastic solids, Eur. J. Mech. A/Solids, 25 (2006) 778-792.

[82] R. W. Ogden, Non-linear elastic deformations, Ellis Horwood: Chichester (1984).

[83] D. A. Prikazchikov, G. A. Rogerson, Some comments on the dynamic properties of anisotropic and strongly anisotropic pre-stressed elastic solids, Int. J. Eng. Sci., 41 (2003) 149-171.

[84] M. Destrade, M. Ottenio, A. V. Pichugin, G. A. Rogerson. Nonprincipal surface waves in deformed incompressible materials, Int. J. Eng. Sci., 42 (2005) 1092-1106.

[85] Y. B. Fu, An Integral Representation of the Surface-Impedance Tensor for Incompressible Elastic Materials, J. Elast., 81 (2005) 75-90.

[86] J. N. Flavin, Surface waves in pre-stressed Mooney material, Q. J. Mech. Appl. Math., 16 (1963) 441-449.

[87] A. L. Gower, M. Destrade, R. W. Ogden, Counter-intuitive results in acousto-elasticity, Wave Motion, 50 (2013) 1218-1228.

[88] J. Merodio, R. W. Ogden, Remarks on instabilities and ellipticity for a fiber reinforced compressible nonlinearly elastic solid under plane deformation, Q. Appl. Math., 63 (2005) 325-333. 
[89] J. Merodio, R. W. Ogden, The influence of the invariant $I_{8}$ on the stress-deformation and ellipticity characteristics of doubly fiberreinforced nonlinearly elastic solids, Int. J. Non-Lin. Mech., 41 (2006) 556-563.

[90] M. Destrade, Elastic interface acoustic waves in twinned crystals, Int. J. Solid. Struct., 40 (2003) 7375-7383.

[91] B. Collet, M. Destrade, Explicit secular equations for piezoacoustic surface waves: Shear-horizontal modes, J. Acoust. Soc. Am., 116 (2004) 3432-3442.

[92] G.A. Holzapfel, R.W. Ogden, On the tension-compression switch in soft fibrous solids, Eur. J. Mech. A/Solids, 49 (2015) 561-569.

[93] W. M. Ewing, W. S. Jardetzky, F.Press, Elastic waves in layered media, New York: McGraw-Hill (1957).

[94] J. D. Achenbach, S. P. Keshava, Free waves in a plate supported by a semi-infinite continuum, J. Appl. Mech., 34 (1967) 397-404.

[95] J. D. Achenbach, H. I. Epstein, Dynamic interaction of a layer and a half-space, J. Eng. Mech., ASCE, 93 (1967) 27-42.

[96] H. F. Tiersten, Elastic surface waves guided by thin films, J. Appl. Phys., 40 (1969) 770-789.

[97] G. W. Farnell, E. L. Adler, Elastic wave propagation in thin layers, Phys. Acoust., 9 (1972) 35-127.

[98] R. W. Ogden, D. A. Sotiropoulos, On interfacial waves in pre-stressed layered incompressible elastic solids, Proc. R. Soc. Lond. A, 450 (1995) 319-341.

[99] S. D. Akbarov, M. Ozisik, Dynamic interaction of a prestressed nonlinear elastic layer and a half-plane, Int. Appl. Mech., 40 (2004) 1056-1063.

[100] A. I. Murdoch, The propagation of surface waves in bodies with material boundaries. J. Mech. Phys. Solids, 24 (1976) 137-146. 
[101] R. W. Ogden, D. J. Steigmann, Plane strain dynamics of elastic solids with intrinsic boundary elasticity and application to surface wave propagation. J. Mech. Phys. Solids, 50 (2002) 1869-1896.

[102] D. J. Steigmann, R. W. Ogden, Plane deformations of elastic solids with intrinsic boundary elasticity, Proc. R. Soc. Lond. A, 453 (1997) 853-877.

[103] M. Hayes, R. S. Rivlin, Surface waves in deformed elastic materials, Arch. Ration. Mech. Anal., 8 (1961) 358-380.

[104] M. A. Biot, Mechanics of Incremental Deformations. New York: John Wiley (1965).

[105] M. A. Dowaikh, R. W. Ogden, On surface waves and deformations in a pre-stressed incompressible elastic solid, IMA J. Appl. Math., 44 (1990) 261-284.

[106] M. A. Dowaikh, R. W. Ogden, On surface waves and deformations in a compressible elastic half-space, Stability Appl. Anal. Cont. Media, 1 (1991a) 27-45.

[107] P. Chadwick, D. A. Jarvis, Surface waves in a pre-stressed elastic body, Proc. R. Soc. Lond. A, 336 (1979) 517-536.

[108] P. Chadwick, The application of the Stroh formalism to prestressed elastic media. Math. Mech. Solids, 2 (1997) 379-403.

[109] M. Shams, R. W. Ogden, On Rayleigh-type surface waves in an initially stressed incompressible elastic solid. IMA J. Appl. Math., 79 (2014) 360-376.

[110] M. Diab, K-S.Kim, Ruga formation instabilities of a graded stiffness boundary layer in a neo-Hookean solid, Proc. R. Soc. Lond. A, 470 (2014) 20140218.

[111] A. Hoger, On the residual stress possible in an elastic body with material symmetry, Arch. Rat. Mech. Anal., 88 (1985) 271-289. 
[112] J. Merodio, R. W. Ogden, J. Rodríguez, The influence of residual stress on finite deformation elastic response, Int. J. Non-Lin. Mech., 56 (2013) 43-49.

[113] M. Shams, M. Destrade, R. W. Ogden, Initial stresses in elastic solids: constituive laws and acoustoelasticity, Wave Motion, 48 (2011) 552-567.

[114] J. Song, H. Jiang, Z. J. Liu, D. Y. Khang, Y. Huang, J. A. Rogers, C. Lu, C. G. Koh, Buckling of a stiff film on a compliant substrate in large deformation Int. J. Solids Structures, 45 (2008) 3107-3121.

[115] J. Y. Sun, S. Xia, M. W. Moon, K. H. Oh, K. S. Kim, Folding wrinkles of a thin layer on a soft substrate, Proc. R. Soc. Lond. A, 468 (2012) 932-953.

[116] Y. Cao, J. W. Hutchinson, Wrinkling phenomena in neo-Hookean film/substrate bilayers, J. Appl. Mech, 79 (2012) 031019 1-9.

[117] L. Jin, A. Takei, J. W. Hutchinson, Mechanics of wrinkle/ridge transitions in thin film/substrate systems, J. Mech. Phys. Solids, $\mathbf{8 1}$ (2015) 22-40.

[118] D. Bigoni, M. Gei, A. B. Movchan, Dynamics of a prestressed stiff layer on an elastic half-space: filtering and band gap characteristics of periodic structural bmodels derived from long-wave asymptotics, $J$. Mech. Phys. Solids, 56 (2008) 2494-2520. 


\section{BIBLIOGRAPHY}


2.1 A surface wave propagates in a principal plane of the prestrain, the plane $x_{2}=0$, but not in a principal direction, its propagation direction makes an angle $\theta$ with the $x_{1}$-axis. The material is reinforced by parallel fibers which make an angle $\alpha$ with the $x_{1}$-axis. . . . . . . . . . . .

2.2 The curves show the dependence of $x=\rho c^{2} / \mu$ obtained using (3.30) on $\theta \in[0 \pi / 2]$, which is the angle that the propagating wave makes with the $x_{1}$-direction. Fibers are parallel to the $X_{1}$-direction, the elastic half-space is initially under uniaxial tension along the $X_{1}$-axis and the strain-energy function is given by (2.33) (dashed line), (2.34) (dash-dot line) and (2.35) (solid line) for $\gamma_{1}=\gamma_{2}=3 / 4$ and $\lambda=1.3 \ldots \ldots \ldots$

2.3 Values of $x=\rho c^{2} / \mu$ obtained using (3.30) vs $\theta \in[0 \pi / 2]$ when the fibre direction makes an angle $\alpha=\pi / 6$ with the $x_{1}$-axis for (2.33) (dash-dot line), (2.34) (dashed line) and (2.35) (solid line), with $\gamma_{1}=\gamma_{2}=3 / 4$ and $\left(\lambda_{1}, \lambda_{2}, \lambda_{3}\right)=$ $(3 / 2,1,2 / 3)$. . . . . . . . . . . . .

3.1 The figure on the left shows at a point $O$ in the free surface of the pre-stressed half space: i) the principal axes of the primary pure homogeneous strain $\left(x_{i}\right.$-axes $)$ ii) the two directions in that configuration characterizing the two families of fibers (given by $\psi$ and $\phi$ ) as well as the fibers of each family (dashed lines) along the depth direction $\left(x_{2}\right.$-axis $)$ and iii) the propagation direction of the wave (given by $\theta$ ). Fibers of each family are located throughout the whole half space and run parallel to each other and perpendicular to the depth direction. The figure on the right is a view from the top. It further clarifies that the angles $\psi$ and $\phi$ are meassured in opposite senses relative to the $x_{1}$-axis. $\ldots \ldots \ldots \ldots \ldots \ldots$ 
3.2 In the two plots, the curves show the dependence of $x=\rho c^{2} / \mu$ on $\theta \in[0, \pi / 2]$ obtained using (3.30) for (3.33), the dotted-dashed curve, (3.34), the thin solid curve, as well as (3.35) and (3.36), the dashed and thick solid curves, respectively. For the different calculations, we have taken, accordingly for each model, $\gamma=\pi / 6, \delta=\pi / 3$, $k_{1}=k_{3}=0.5 \mu, k_{2}=0.5$. The principal stretches are $\lambda_{1}=\lambda=1.2, \lambda_{2}=1, \lambda_{3}=1 / \lambda_{1}$ (left-hand plot); $\lambda_{1}=1.3, \lambda_{2}=1$ and $\lambda_{3}=1 / \lambda_{1}$ (right-hand plot). . . . .

3.3 The curves show in the two plots the dependence of $x=\rho c^{2} / \mu$ on $\theta \in[0, \pi / 2]$ as given by (3.30) for (3.33), the dotted-dashed curve, as well as (3.35) and (3.36), the dashed and thick solid curves, respectively. The parameters of the different models have been taken as $\gamma=\pi / 4, \delta=\pi / 4, k_{1}=k_{3}=0.5 \mu$ and $k_{2}=0.5$. The principal stretches are $\lambda_{1}=1.2, \lambda_{2}=1, \lambda_{3}=1 / \lambda_{1}$ (left-hand plot) and b) $\lambda_{1}=1.3, \lambda_{2}=1, \lambda_{3}=1 / \lambda_{1}$ (right-hand plot). Results for the neo-Hookean model (3.34), the thin solid curve, are also shown for comparison.

3.4 Corresponding plots to the ones given in Figure 3.2 for $\gamma=$ $\pi / 6$ and $\delta=\pi / 4 \ldots \ldots \ldots \ldots \ldots$

3.5 Corresponding plots to the ones given in Figure 3.2 for $\gamma=$ $\pi / 6$ and $\delta=\pi / 6 \ldots \ldots \ldots \ldots \ldots$

3.6 Under uniaxial tension along the $X_{1}$-axis with $\gamma=\delta=\pi / 4$ and waves propagating along the $x_{1}$-axis, the Figure shows the dependence of $x=\rho c^{2} / \mu$ on $\lambda$ as given by (3.30) for (3.35), the dotted curve, and (3.36), the dashed curve. Results for the neo-Hookean model (3.34), the solid curve, are also shown for comparison. The parameters for the different models are $k_{1}=0.5 \mu$ and $k_{2}=0.5$. . . . . . .

3.7 Under uniaxial tension along the $X_{1}$-axis with $\lambda_{1}=1.2$, $\lambda_{2}=1, \lambda_{3}=1 / \lambda_{1}$ and waves propagating along the $x_{1}$-axis, the figures shows values of $x=\rho c^{2} / \mu$ vs $\gamma=\delta \in[0, \pi / 2]$, (the angle that each family of fibers makes with the $X_{1}$-direction) as given by (3.30) for (3.33), (3.35) and (3.36), dotted-dashed, dashed and thick solid curves, respectively. The values of the parameters used in the calculations are $k_{1}=k_{3}=0.5 \mu$ and $k_{2}=0.5$. Results for the neo-Hookean model (3.34), the thin solid curve, are also shown for comparison. . . . . . . . . 
4.1 Plots of the curves $\epsilon=0$ (dashed curves) and $\xi=0$ (continuous curves) in $(\lambda, \bar{\tau})$ space for $\bar{\mu}=$ (a) 0.5 , (c) 1 , (e) 5. The + sign indicates the regions of values of $\lambda$ and $\bar{\tau}$ for which surface waves exist and where $\xi>0$. Plots of the curves $\eta_{\mathrm{L}}=0$ (dashed) and $\xi=0$ (continuous) for $\bar{\mu}=(\mathrm{b})$ -0.5, (d) -1 , (f) -5 . The + sign indicates the regions of values of $\lambda$ and $\bar{\tau}$ for which surface waves exist and where $\eta_{\mathrm{L}}>0$ and $\xi>0 \ldots \ldots \ldots \ldots$

4.2 Plots of $\zeta=\rho c^{2} / \mu$ against $k h$ with $\lambda=\lambda^{*}=1, \bar{\tau}=\bar{\tau}^{*}=0$ and $r=0.2$ (thick continuous curves), $r=1$ (continuous curves), $r=5$ (dashed curves): (a) $R=0.1$; (b) $R=0.4$; (c) $R=0.6$; (d) $R=0.9$; (e) $R=0.95$; (f) $R=1$. . . .

4.3 Plots of $\zeta=\rho c^{2} / \mu$ against $k h$ with $\lambda=\lambda^{*}=1, \bar{\tau}=\bar{\tau}^{*}=0$ and $r=0.2$ (thick continuous curves), $r=1$ (continuous curves), $r=5$ (dashed curves): (a) $R=1$; (b) $R=1.25$; (c) $R=1.6$; (d) $R=2 \ldots \ldots \ldots \ldots$

4.4 In each panel $\zeta=\rho c^{2} / \mu$ is plotted against $k h$ for $R=1$ and $r=0.2$ (thick continuous curves), $r=1$ (continuous curves), $r=5$ (dashed curves): (a) $\lambda=\lambda^{*}=1, \bar{\tau}=-0.2, \bar{\tau}^{*}=0 ; \quad(\mathrm{b})$ $\lambda=\lambda^{*}=1, \bar{\tau}=0, \bar{\tau}^{*}=-0.2 ; \quad(\mathrm{c})$ $\lambda=0.9, \lambda^{*}=1, \bar{\tau}=0, \bar{\tau}^{*}=0 ; \quad(\mathrm{d})$ $\lambda=1, \lambda^{*}=0.9, \bar{\tau}=0, \bar{\tau}^{*}=0 . \ldots \ldots \ldots$

4.5 In each panel $\zeta=\rho c^{2} / \mu$ is plotted against $k h$ for $R=1$ and $r=0.2$ (thick continuous curves), $r=1$ (continuous curves), $r=5$ (dashed curves):(a) $\lambda=\lambda^{*}=1, \bar{\tau}=1, \bar{\tau}^{*}=0$; (b) $\lambda=\lambda^{*}=1, \bar{\tau}=0, \bar{\tau}^{*}=1$; (c) $\lambda=1.2, \lambda^{*}=1, \bar{\tau}=$ $0, \bar{\tau}^{*}=0 ;$ (d) $\lambda=1, \lambda^{*}=1.4, \bar{\tau}=0, \bar{\tau}^{*}=0 \ldots \ldots$. . .

4.6 Plots of $\zeta$ against $k h$ for $\lambda=\lambda^{*}=1, R=1, \bar{\mu}=0, \bar{\tau}=1$, $\bar{\mu}^{*}=-1.5, \bar{\tau}^{*}=1$ : (a) $r=0.1$; (b) $r=10 \ldots$. . . . . .

4.7 Plots of $\zeta$ against $k h$ : (a) $\lambda=1, \lambda^{*}=0.8, R=1$, $\bar{\mu}=-1.5, \bar{\tau}=-0.5, \bar{\mu}^{*}=0, \bar{\tau}^{*}=-0.5$, and $r=0.2,1,2$ corresponding to the thick continuous, continuous and dashed curves, respectively; (b) $\lambda=1.2, \lambda^{*}=0.8, R=0.8$, $\bar{\mu}=-1, \bar{\tau}=1, \bar{\mu}^{*}=0, \bar{\tau}^{*}=-0.5$, and $r=0.2,0.5,2$ corresponding to the thick continuous, continuous and dashed curves, respectively. . . . . . . . . . . . .

4.8 Plots of $\bar{\tau}^{*}$ against $k h$ for $\bar{\mu}=\bar{\mu}^{*}=0, \lambda=\lambda^{*}=1, \zeta=0$, $R=1$ : (a) $\bar{\tau}=-0.5$; (b) $\bar{\tau}=0.5$. In each of (a) and (b) curves are shown for $r=0.5,1.5,3,10$, respectively the thick continuous, dashed, continuous and thick dashed curves. 70

4.9 Plots of $\lambda$ vs $k h$ for $\bar{\mu}=\bar{\mu}^{*}=0, \bar{\tau}=\bar{\tau}^{*}=0, \zeta=0, R=$ $1, r=\mu^{*} / \mu=0.2,1,3.8,6$ in each plot. The panels (a), (b), (c), (d) correspond to $\lambda^{*}=1.4,0.854,0.85,0.8$, respectively. 
